\title{
DESIGN OF A LOW-COST DATA ACQUISITION SYSTEM FOR ROTORDYNAMIC DATA COLLECTION
}

\author{
A Thesis \\ presented to \\ the Faculty of California Polytechnic State University, \\ San Luis Obispo
}

\author{
In Partial Fulfillment \\ of the Requirements for the Degree \\ Master of Science in Mechanical Engineering
}

by

Gregory Samuel Pellegrino

March 2019 
(C) 2019

Gregory Samuel Pellegrino

ALL RIGHTS RESERVED

ii 
TITLE: Design of a Low-Cost Data Acquisition System for Rotordynamic Data Collection

AUTHOR: Gregory Samuel Pellegrino

DATE SUBMITTED: $\quad$ March 2019

COMMITTEE CHAIR: Xi Wu, Ph.D.

Professor of Mechanical Engineering

COMMITTEE MEMBER: John R. Ridgely, Ph.D.

Professor of Mechanical Engineering

COMMITTEE MEMBER: William R. Murray, Ph.D.

Professor of Mechanical Engineering 


\begin{abstract}
Design of a Low-Cost Data Acquisition System for Rotordynamic Data Collection Gregory Pellegrino
\end{abstract}

A data acquisition system (DAQ) was designed based on the use of a STM32 microcontroller. Its purpose is to provide a transparent and low-cost alternative to commercially available DAQs, providing educators a means to teach students about the process through which data are collected as well as the uses of collected data. The DAQ was designed to collect data from rotating machinery spinning at a speed up to 10,000 RPM and send this data to a computer through a USB 2.0 full-speed connection. Multitasking code was written for the DAQ to allow for data to be simultaneously collected and transferred over USB. Additionally, a console application was created to control the DAQ and read data, and MATLAB code written to analyze the data. The DAQ was compared against a custom assembled National Instruments CompactDAQ system. Using a Bentley-Nevada RK 4 Rotor Kit, data was simultaneously collected using both DAQs. Analysis of this data shows the capabilities and limitations of the low cost DAQ compared to the custom CompactDAQ.

Key words: data acquisition, test and measurement device, analog to digital converter, real time operating system, finite state machine, ground loop 


\section{ACKNOWLEDGMENTS}

I would like to thank the members of my committee: Dr. Xi Wu, Dr. John Ridgely, and

Dr. William R. Murray. They provided me with the knowledge and resources that made this thesis possible.

I would also like to thank my family for all the support that they have provided me during my time at Cal Poly. 


\section{TABLE OF CONTENTS}

Page

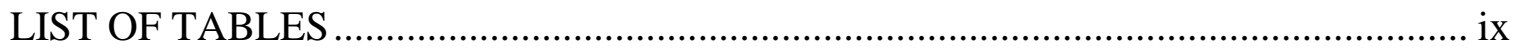

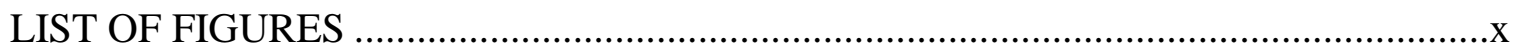

\section{CHAPTER}

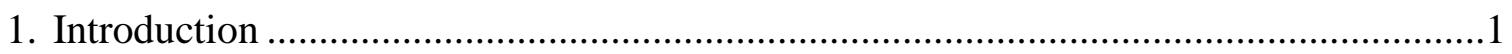

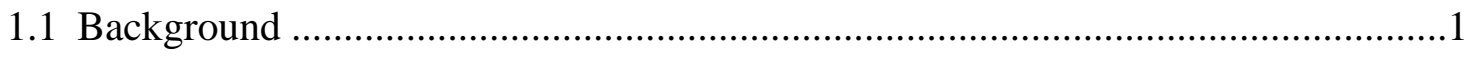

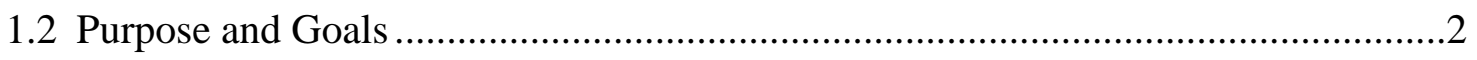

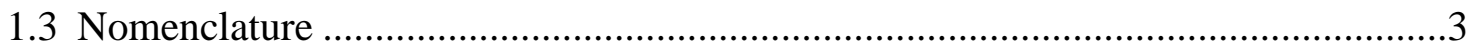

2. Bentley-Nevada Rotor Kit...........................................................................

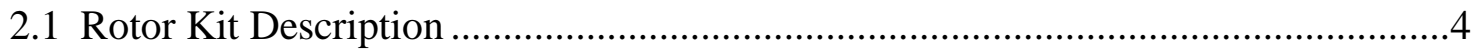

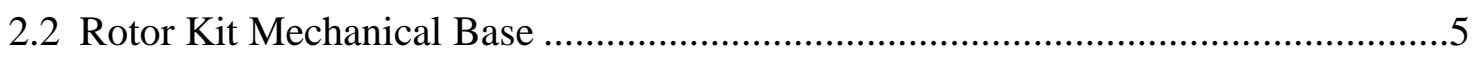

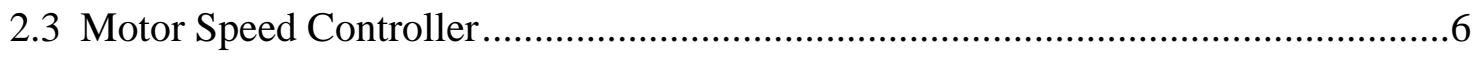

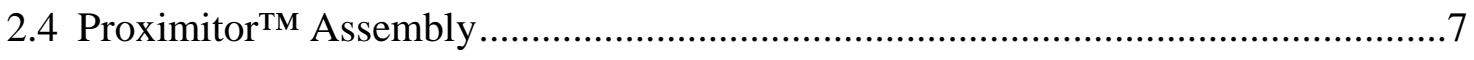

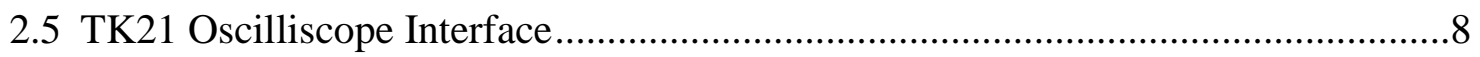

3. National Instruments CompactDAQ with MATLAB .................................................

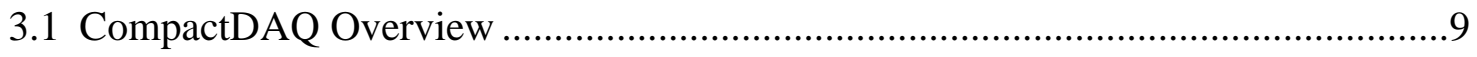

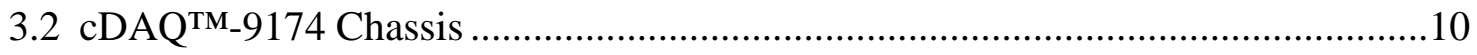

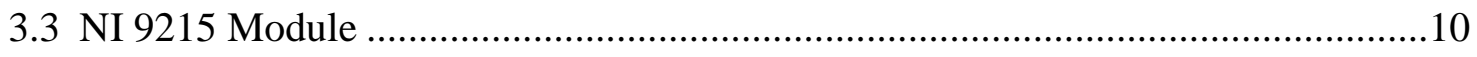

3.4 MATLAB Rotor Kit App …………………...............................................11

4. STM32 USB Data Acquisition System Hardware …….................................................13

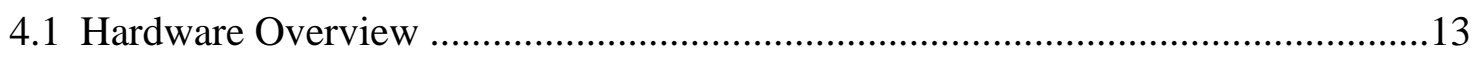

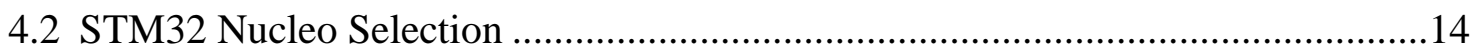


4.3 Data Acquisition Expansion Board Design ..................................................15

4.3.1 USB 2.0 Full-Speed Implementation ..........................................................16

4.3.2 Analog to Digital Converter Implementation ..........................................18

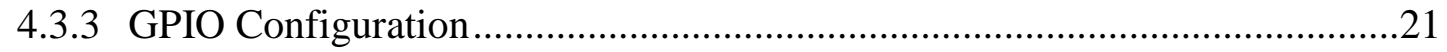

4.3.4 Board Design Considerations ................................................................22

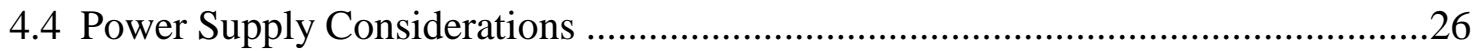

5. STM32 USB Data Acquisition System Software ..................................................28

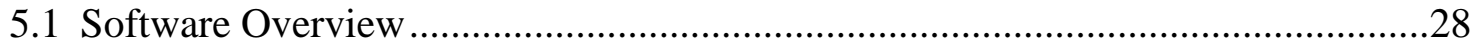

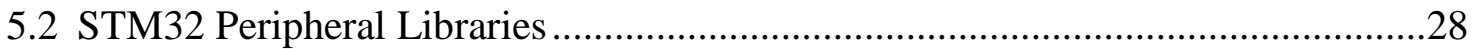

5.2.1 USB Test and Measurement Class Device Library ...................................29

5.2.2 AD760x Analog to Digital Converter Library ............................................30

5.3 Data Acquisition System RTOS and Finite State Machines ................................30

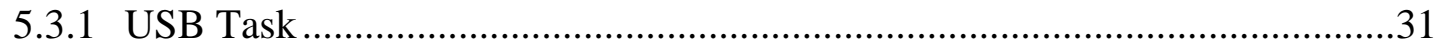

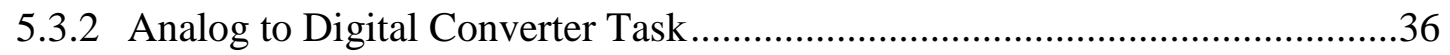

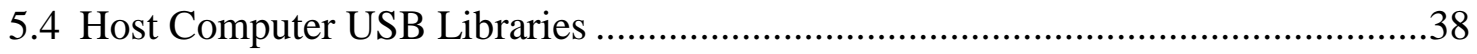

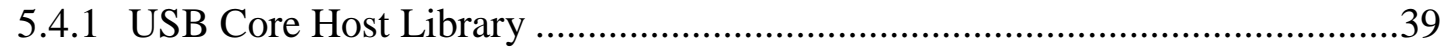

5.4.2 USB Test and Measurement Class Host Library .......................................39

5.5 Rotordynamic Data Acquisition and Processing .............................................40

5.5.1 Data Acquisition Console Application ...............................................40

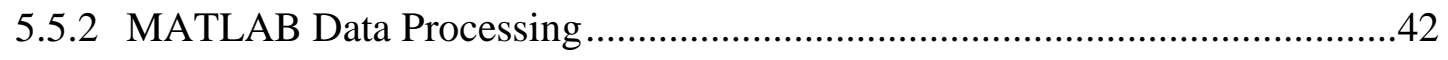

6. NI CompactDAQ and STM32 USB DAQ Attributes Comparison............................44

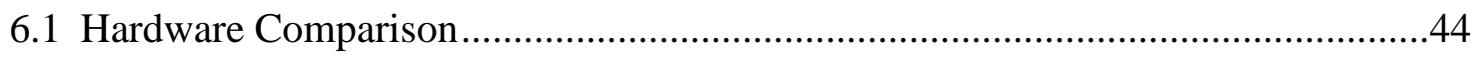

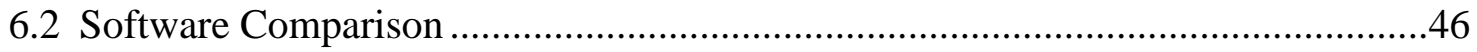


7. Experiments using the STM32 USB Data Acquisition System

7.1 STM32 USB Data Acquisition System Initial Measurements Verification

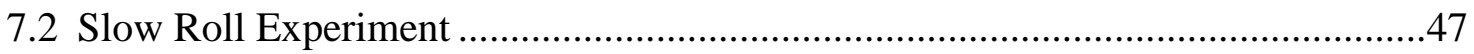

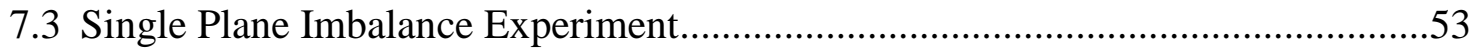

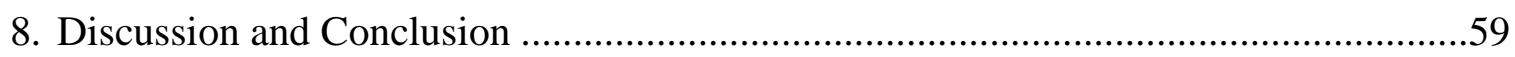

8.1 Data Acquisition System Design Summary ......................................................59

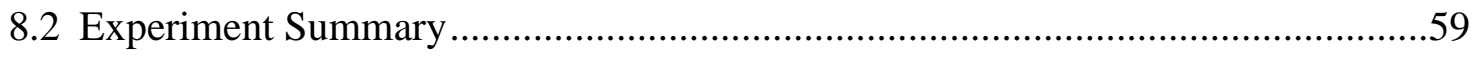

8.3 Conclusion and Future Recommendations ...................................................60

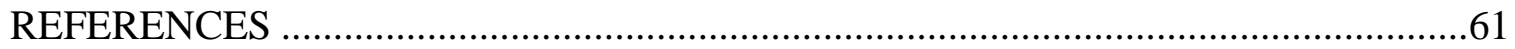

\section{APPENDICIES}

A: Data Acquisition Expansion Board Schematic .............................................63

B: Data Acquisition Extension Board Copper Fill ................................................. 71

C: Data Acquisition Expansion Board Bill of Materials and Notes............................75

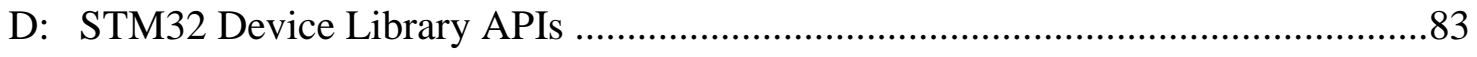

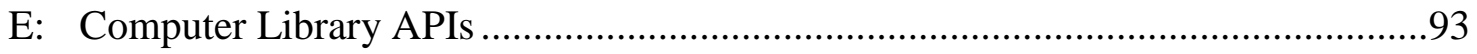

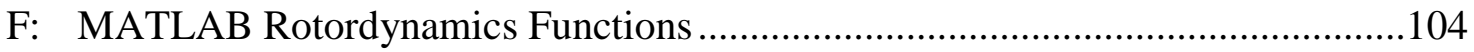

G: STM32 USB Data Acquisition System Task and State Diagrams ......................122

H: How to Use the STM32 USB Data Acquisition System ..................................125 


\section{LIST OF TABLES}

Table

Page

1. List of abbreviations used throughout the paper. .............................................. 3

2. A comparison between the Analog Devices AD760x family of ADCs. .................. 19

3. A description of all commands used by the STM32 USB DAQ........................... 33

4. Hardware comparison between the NI CompactDAQ and the STM32 USB

DAQ. Although the analog to digital converter used by the STM32 USB DAQ is capable of a sampling rate of $200 \mathrm{kSPS}$ per channel, this sampling rate cannot be achieved due to board design limitations. 


\section{LIST OF FIGURES}

Figure

Page

1. One of the RK 4 Rotor Kits used in the Cal Poly Vibrations Lab.............................4

2. The mechanical base of an RK 4 Rotor Kit. The $\mathrm{X}$ and $\mathrm{Y}$ proximity probes are located next to a rotor mass to measure its vibration amplitude.

3. The Rotor Kit Motor Speed Controller. The maximum speed of the motor is set to approximately $3000 \mathrm{RPM}$, and its ramp rate is set to $4000 \mathrm{RPM} / \mathrm{min}$.

4. The front (left) and back (right) sides of the Rotor Kit Proximitor ${ }^{\mathrm{TM}}$ Assembly........7

5. One of the TK21 Oscilloscope Interfaces used with the Rotor Kits in the Cal Poly Vibrations Lab.

6. The CompactDAQ system used in the Cal Poly Vibrations Lab. It is assembled from a cDAQ ${ }^{\mathrm{TM}}-9174$ chassis and two NI 9215 with BNC modules

7. Top (left) and front (right) views of The CompactDAQ system. The two unused module ports can be seen on the left. The side of the NI 9215 modules, shown on the right, includes diagrams showing the connection of the BNC connectors to the ADC differential inputs.

8. The MATLAB Rotor Kit App interface. Data is collected from the CompactDAQ by entering the desired options and clicking the "Run" button

9. The STM32 USB Data Acquisition System. It is assembled from an STM32 Nucleo development board and a Data Acquisition Expansion Board.

10. The top (left) and bottom (right) of the Data Acquisition Expansion Board. .15

11. Calculation of the required USB data line trace width using the Edge Coupled Microstrip Impedance Calculator from EEWeb.com. 
12. Calculation of the trace spacing necessary to limit crosstalk using the Microstrip Crosstalk Calculator from EEWeb.com.

13. Physical comparison between the NI CompactDAQ (left) and the STM32 USB DAQ (right). .44

14. Test measurement of the signal generator output using the STM32 USB DAQ. The signals measured, from top to bottom, are a sine wave, a square wave, a triangle wave, and a sawtooth wave. The dashed lines at $-1,0$, and 1 volts provide a frame of reference.

15. Rotor Kit proximity sensor connections to both the CompactDAQ and the STM32 USB DAQ.

16. Raw voltage data collected from the RK 4 Rotor Kit using the STM32 USB DAQ and the CompactDAQ. Pulses occur at regular intervals in the STM32 USB DAQ data, but not the CompactDAQ data. .50

17. Raw voltage data collected from the RK 4 Rotor Kit using the STM32 USB DAQ and the CompactDAQ. The pulses previously seen in the STM32 USB DAQ data disappear when the system is isolated from all sources except the sensor inputs.

18. Image of the HitLetgo (left), SMAKN (middle), and EZSync (right) USB isolators used with the STM32 USB DAQ

19. Slow roll test results using the HiLetgo (top), SMAKN (middle), and EZSync (bottom) USB isolators. Each isolator was compared against the CompactDAQ. ...54 
20. Bode plots showing the amplitude and phase lag in the horizontal direction.

These results were analyzed using data collected from the CompactDAQ and the

STM32 USB DAQ during the single plane imbalance experiment.

21. Uncompensated polar plot showing amplitude with respect to phase lag in the horizontal direction.

22. Full spectrum cascade plots produced from data collected by the STM32 USB

DAQ (top) and the CompactDAQ (bottom). .58

A1. Schematic of the Data Acquisition Expansion Board headers.

A2. Schematic of the Data Acquisition Expansion Board USB port. .64

A3. Schematic of the Data Acquisition Expansion Board current limiter. .65

A4. Schematic of the Data Acquisition Expansion Board ADC. .66

A5. Schematic of the Data Acquisition Expansion Board voltage reference.

A6. Schematic of the Data Acquisition Expansion Board ADC configuration jumpers. .68

A7. Schematic of the Data Acquisition Expansion Board ADC solder bridges. 69

A8. Schematic of the Data Acquisition Expansion Board BNC analog inputs. .70

B1. Top side layer copper fill of the Data Acquisition Expansion Board. 71

B2. Top inner layer copper fill of the Data Acquisition Expansion Board. .72

B3. Bottom inner layer copper fill of the Data Acquisition Expansion Board.

B4. Bottom side layer copper fill of the Data Acquisition Expansion Board.

G1. STM32 USB Data Acquisition System task diagram. The USB task has two timing requirements dependent on the state it is in. 
G2. STM32 USB Data Acquisition System USB task state diagram. States 0 and 1 have a timing requirement of $100 \mathrm{~ms}$, while state 2 has a timing requirement of $50 \mu \mathrm{s}$.

G3. STM32 USB Data Acquisition System ADC task state diagram.............................124

H1. The console application used to collect data from the STM32 USB DAQ.............128 


\section{Chapter 1: Introduction}

\subsection{Background}

It is often necessary to observe and analyze the characteristics of a physical system. Data acquisition is the process of sampling and recording one or more physical characteristics

of a system. These characteristics, often referred to as variables, would allow the system to be analyzed. Measurement of these variables is useful for purposes such as maintaining proper operation of machinery or researching physical phenomena. A data acquisition system (often abbreviated as DAQ or DAS) is an instrument used to measure and record these variables. Data acquisition systems come in many different formats, from electromechanical systems that plot recorded data directly on a roll of paper, to electronic systems that send data directly to a computer [1].

Many mechanical systems incorporate rotating machinery. It is important to monitor the vibration produced by this machinery, as it can cause fatigue failure due to the periodic stress or even wear and damage due to unwanted contact between parts. In large systems, even vibrations on the scale of a few thousandths of an inch, mils, can be unacceptable [2]. As such, it is crucial to use high accuracy data acquisition systems to monitor these vibrations. Commercially available equipment, such as the Bentley Nevada ADRE (Automated Diagnostics for Rotating Equipment) System, offer the accuracy necessary for monitoring this machinery, and are useful in industrial settings; however, the high cost of such equipment makes them less suitable for educational purposes. The National Instruments CompactDAQ chassis and compatible modules, when paired with 
MATLAB, provides a much lower cost alternative [3], but still comes at a steep price for most educators.

\subsection{Purpose and Goals}

The purpose of this thesis was to create a low-cost alternative to existing data acquisition systems. This system is designed transparently, allowing for design modifications to easily be made by others. The low cost and transparency of this DAQ is very useful in educational settings for teaching about the means through which data is acquired as well as the uses of such data. Although useable with any system, the DAQ is designed to collect data from rotating machinery for analysis.

This DAQ was compared against a custom-built National Instruments CompactDAQ system. The CompactDAQ system, assembled from a cDAQ ${ }^{\mathrm{TM}}-9174$ chassis and two NI 9215 modules, utilizes two 16-bit analog to digital converters, each with four analog input channels capable of simultaneously sampling analog signals within a \pm 10 volt range. Each channel has a differential input that can be sampled at a rate of $100 \mathrm{kSPS}$ per channel and can withstand analog signals of up to \pm 30 volts. Collected data are sent through the USB high-speed port integrated in the chassis. The previously developed MATLAB Rotor Kit App uses this CompactDAQ system to collect and process rotordynamic data in real time [3].

It was desired that the design of the low cost DAQ provided similar capabilities to that of the custom CompactDAQ described above. The main design goals included the capability to measure analog signals within a \pm 10 volt range, collect data at a fast enough rate to measure a rotor spinning at a speed of up to $10,000 \mathrm{RPM}$, and send data to a 
computer through at least a USB full-speed connection. Additional goals, though not necessary for the use of the DAQ, were to match or exceed the listed capabilities of the custom CompactDAQ.

\subsection{Nomenclature}

The following abbreviations seen in Table 1 are used throughout this paper. The measurement units additionally utilize metric unit prefixes.

Table 1. List of abbreviations used throughout the paper.

\begin{tabular}{|l|l|}
\hline Abbreviation & Description \\
\hline A & Amp \\
\hline ADC & Analog to Digital Converter \\
\hline API & Application Program Interface \\
\hline b & Bit \\
\hline BSP & Board Support Package \\
\hline CSV & Comma-Separated Values \\
\hline EMI & Electromagnetic Interference \\
\hline EOM & End of Message \\
\hline ESD & Electrostatic Discharge \\
\hline GPIO & General Purpose IO \\
\hline HAL & Hardware Abstraction Layer \\
\hline Hz & Hertz \\
\hline IO & Input/Output \\
\hline SPS & Samples Per Second \\
\hline LED & Light Emitting Diode \\
\hline mils & Thousandth of an Inch \\
\hline min & Minutes \\
\hline RPM & Revolutions Per Minute \\
\hline RTOS & Real Time Operating System \\
\hline s, sec & Second \\
\hline SPI & Serial Peripheral Interface \\
\hline SPL & Standard Peripheral Library \\
\hline USB & Universal Serial Bus \\
\hline USBTMC & USB Test and Measurement Class \\
\hline V & Volt \\
\hline in & Inch \\
\hline
\end{tabular}




\section{Chapter 2: Bentley-Nevada Rotor Kit}

\section{$\underline{2.1}$ Rotor Kit Description}

The RK 4 Rotor Kit, developed by Bentley-Nevada, is a test mechanism used to simulate rotating machinery. The design of the Rotor Kit allows for easy configuration and can therefore be set up to produce a variety of different behaviors. This kit can be used in education environments to teach students about rotating machinery behaviors and malfunctions, or it can be used for research purposes to simulate actual machinery. As can be seen in Figure 1, the individual parts of the Rotor Kit include the mechanical base, the Proximitor ${ }^{\mathrm{TM}}$ Assembly and the motor speed controller [4]. Additionally, two TK21 Oscilloscope Interfaces are used with the Rotor Kit. Other components, such as the Oil Whirl/Whip Kit, can also be used with the Rotor Kit to produce additional rotordynamic behaviors; however, they were not used for the experiments discussed in this paper.

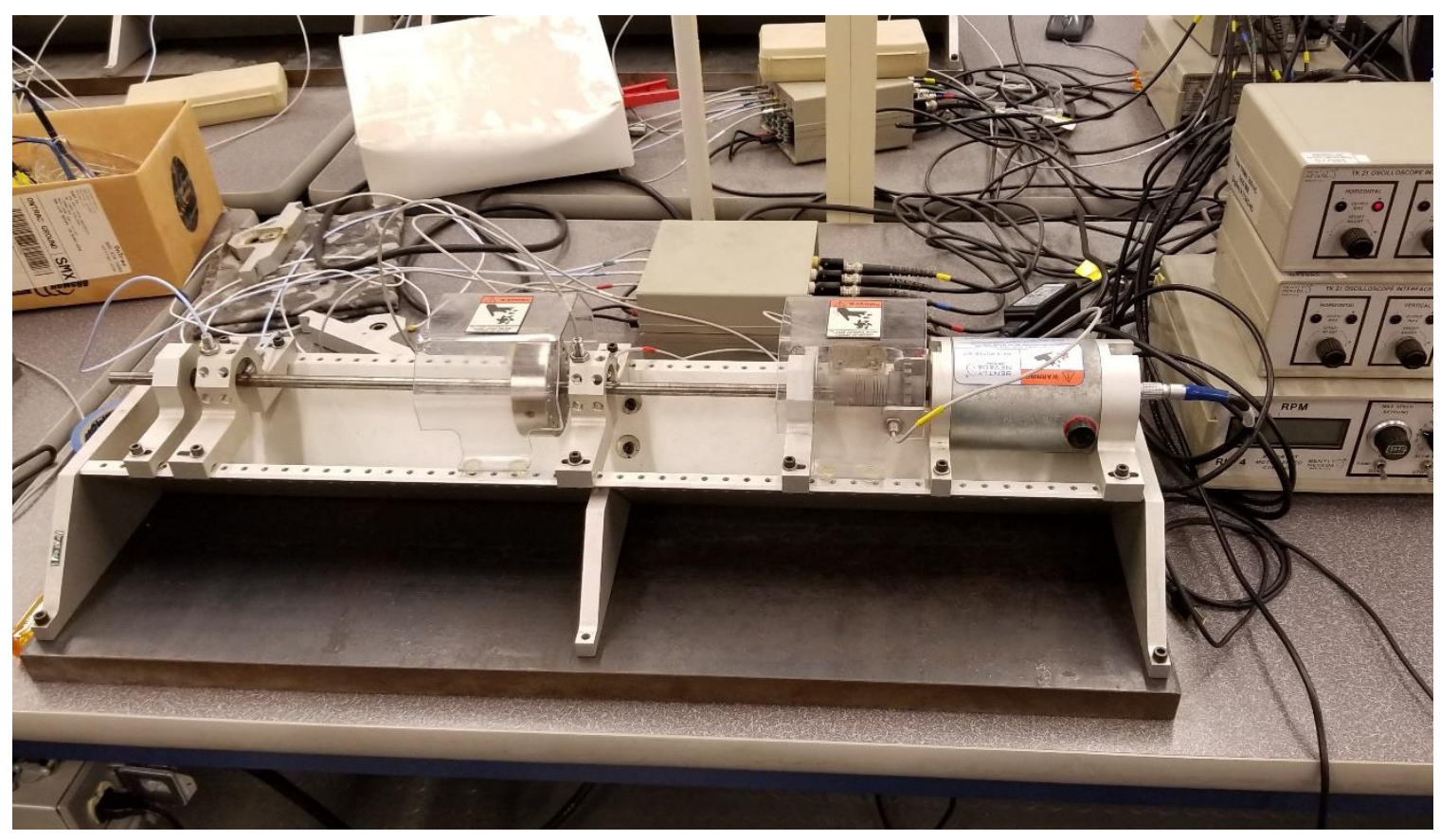

Figure 1. One of the RK 4 Rotor Kits used in the Cal Poly Vibrations Lab. 


\subsection{Rotor Kit Mechanical Base}

The mechanical base of the Rotor Kit is the physical mechanism that is used to simulate rotating machinery. The mechanical base is controlled by the motor speed controller and is capable of spinning at speeds up to 10,000 RPM. As can be seen in Figure 2, the basic mechanical base assembly consists of the base, motor, rotor shaft, journal bearing blocks, six proximity sensors and their mounts, and safety covers [4].

One of the six proximity sensors is used for speed control by the motor speed controller (see section 2.3). Of the remining five, one is used for the Keyphasor ${ }^{\mathrm{TM}}$, two are used horizontal (X) amplitude measurement, and two are used vertical (Y) amplitude measurement. The Keyphasor is used to indicate each rotation of the shaft, while the $\mathrm{X}$ and Y sensors are each paired to allow both horizontal and vertical amplitude measurements to be measured from the same location on the rotor shaft. The Keyphasor and two pairs of $\mathrm{X}$ and $\mathrm{Y}$ proximity sensors can be used by an oscilloscope or by a data acquisition system, allowing for an experimental setup to be analyzed.

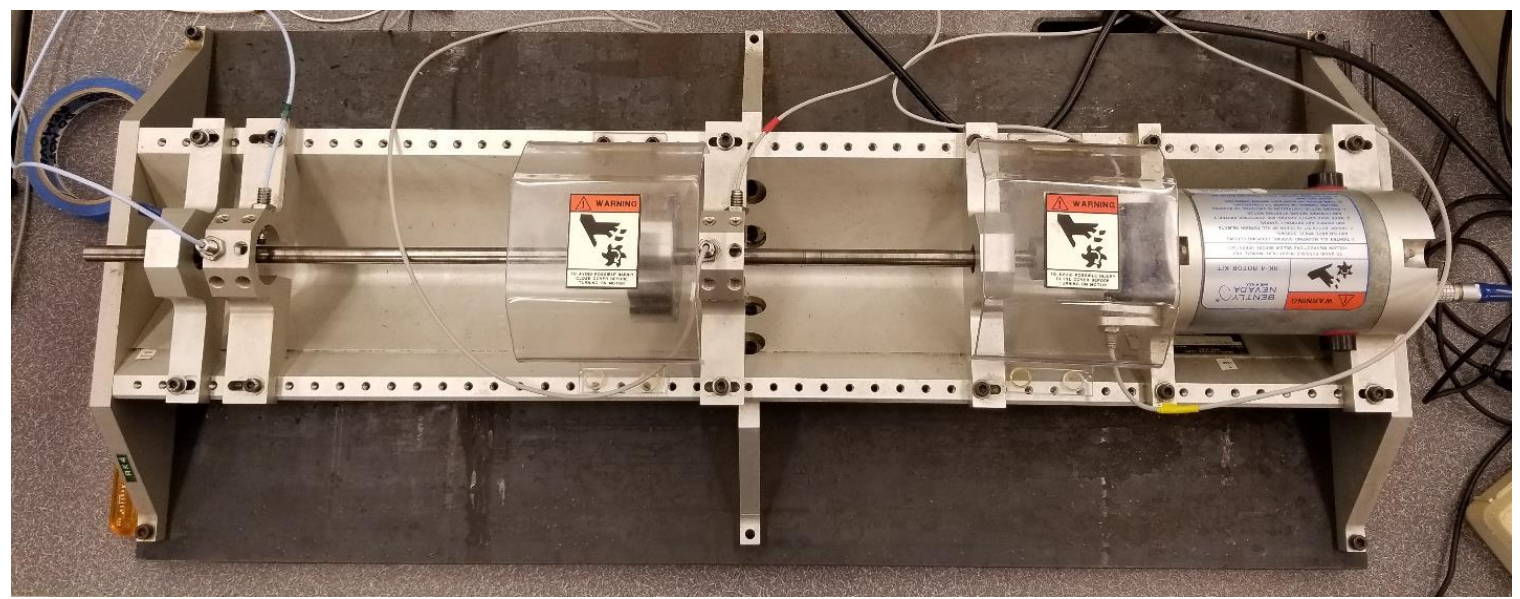

Figure 2. The mechanical base of an RK 4 Rotor Kit. The $\mathrm{X}$ and $\mathrm{Y}$ proximity probes are located next to a rotor mass to measure its vibration amplitude. 
The screw holes in the base, as seen in Figure 2, allow components to be adjusted for different experiments. For example, the journal bearing blocks can easily be moved to different locations to simulate different rotor shaft lengths. Similarly, the proximity sensor mounts can be moved to allow the vibration amplitude to be measured at location of interest along the shaft, such as the location of a rotor mass.

\section{$\underline{2.3 \text { Motor Speed Controller }}$}

The motor speed controller is used to control the Rotor Kit mechanical base. The motor speed controller uses the motor speed proximity sensor to measure the speed of the motor and control the motor accordingly. As can be seen in Figure 3, the motor speed controller controls the motor using two knobs and two switches. The left knob is used to control maximum speed of the motor. The number shown on the knob is in units of 10 RPMs; for example, the value shown in the figure is approximately 300 , indicating a maximum set motor speed of approximately 3000 RPM. Using this knob, the maximum motor

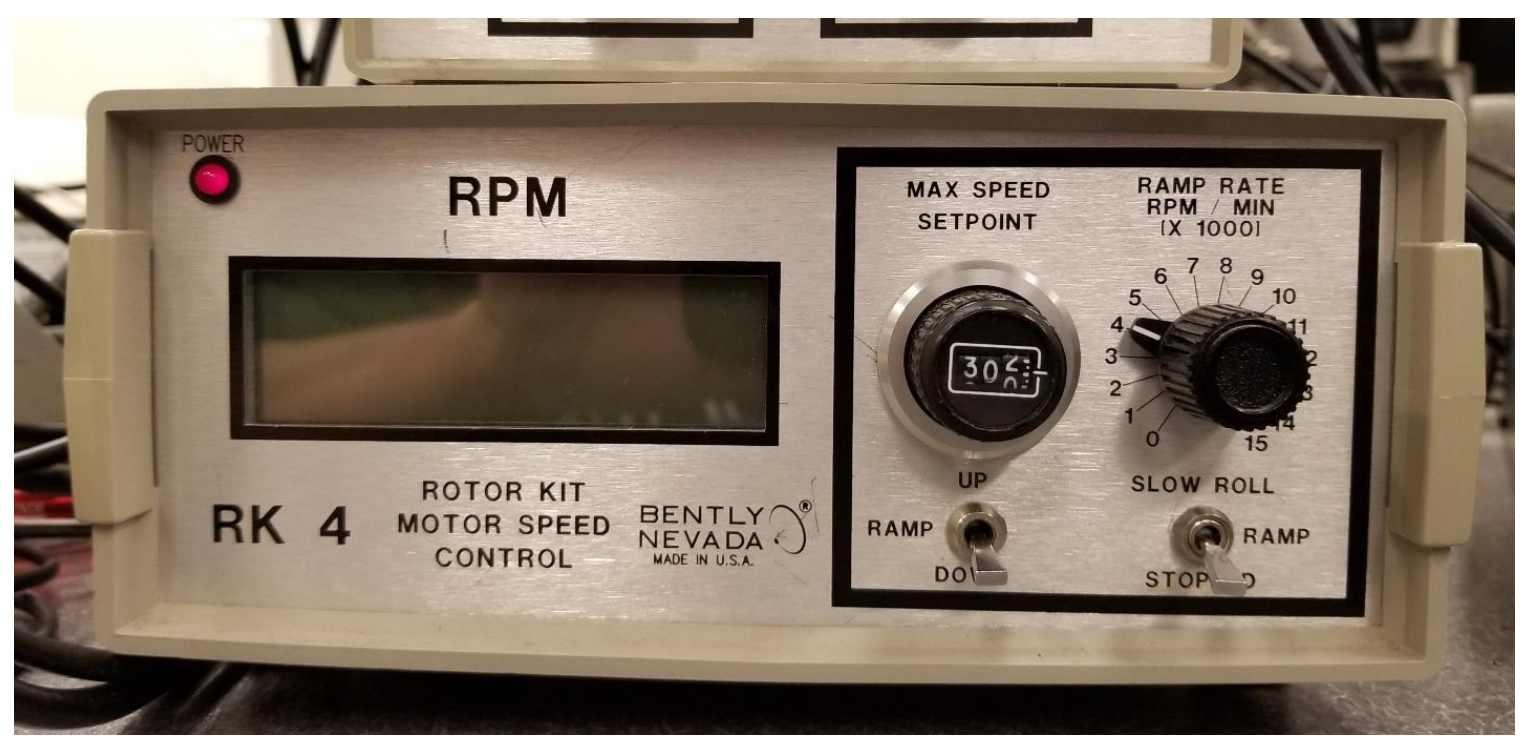

Figure 3. The Rotor Kit Motor Speed Controller. The maximum speed of the motor is set to approximately $3000 \mathrm{RPM}$, and its ramp rate is set to $4000 \mathrm{RPM} / \mathrm{min}$. 
speed can be set to a value up to 10,000 RPM. The other knob is used control the ramp rate of the motor. As can be seen in the figure, the motor ramp rate can be set to a value up to $15,000 \mathrm{RPM} / \mathrm{min}$ [4]. The left switch sets the motor ramp direction to either up or down; however, this switch is only active when the motor controller is in ramp mode. The right switch, a three-position switch, sets the motor control mode to either slow roll, ramp, or stopped. When the motor control is in ramp mode, the motor ramps in the direction set by the left switch.

\subsection{Proximitor ${ }^{\mathrm{TM}}$ Assembly}

The Rotor Kit Proximitor Assembly provides power for the two X, two Y, and one Keyphasor ${ }^{\mathrm{TM}}$ proximity sensors. The Proximitor Assembly itself is powered by the Motor Speed Controller. As can be seen in Figure 4, the proximity sensor inputs are plugged into the back side of the Proximitor Assembly, while the outputs are connected to the front side. The Proximitor Assembly outputs are buffered and produce readings at a level of $200 \mathrm{mV} / \mathrm{mil} \mathrm{[4].}$
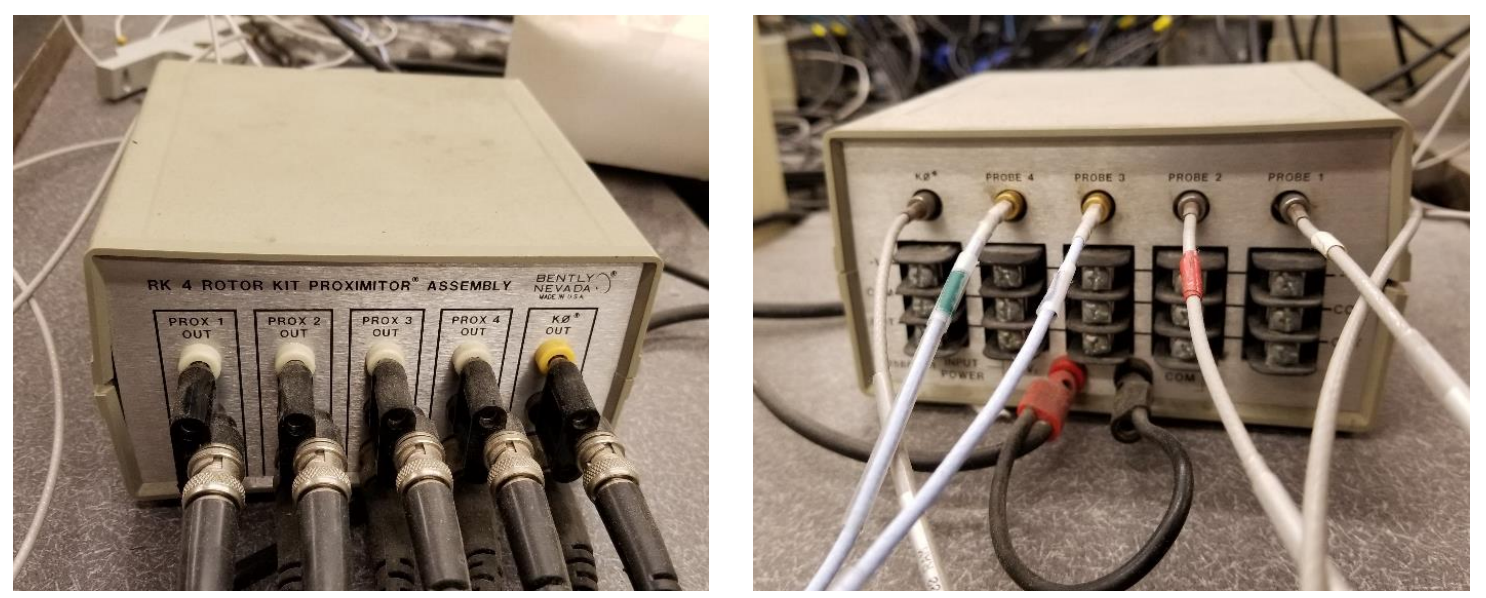

Figure 4. The front (left) and back (right) sides of the Rotor Kit Proximitor ${ }^{\mathrm{TM}}$ Assembly. 


\subsection{TK21 Oscilliscope Interface}

The TK21 Oscilliscope Interface is used to offset the $\mathrm{X}$ and $\mathrm{Y}$ proximity sensor readings. This allows the outputs of the sensors to be offset to zero while the Rotor Kit is running at a slow roll speed, which aids in the creation of orbit plots [5]. Two are used with each Rotor Kit, one for each set of $\mathrm{X}$ and $\mathrm{Y}$ proximity sensors. As can be seen in Figure 5, the offset for each proximity sensor is adjusted using a dial. Each proximity sensor has two indicator lights that are used to indicate when the output is biased. When the output is negatively biased, the negative bias light illuminates. Similarly, when the output is positively biased, the positive bias light illuminates. When there is no bias, and the output reading is approximately zero, neither light is illuminated.

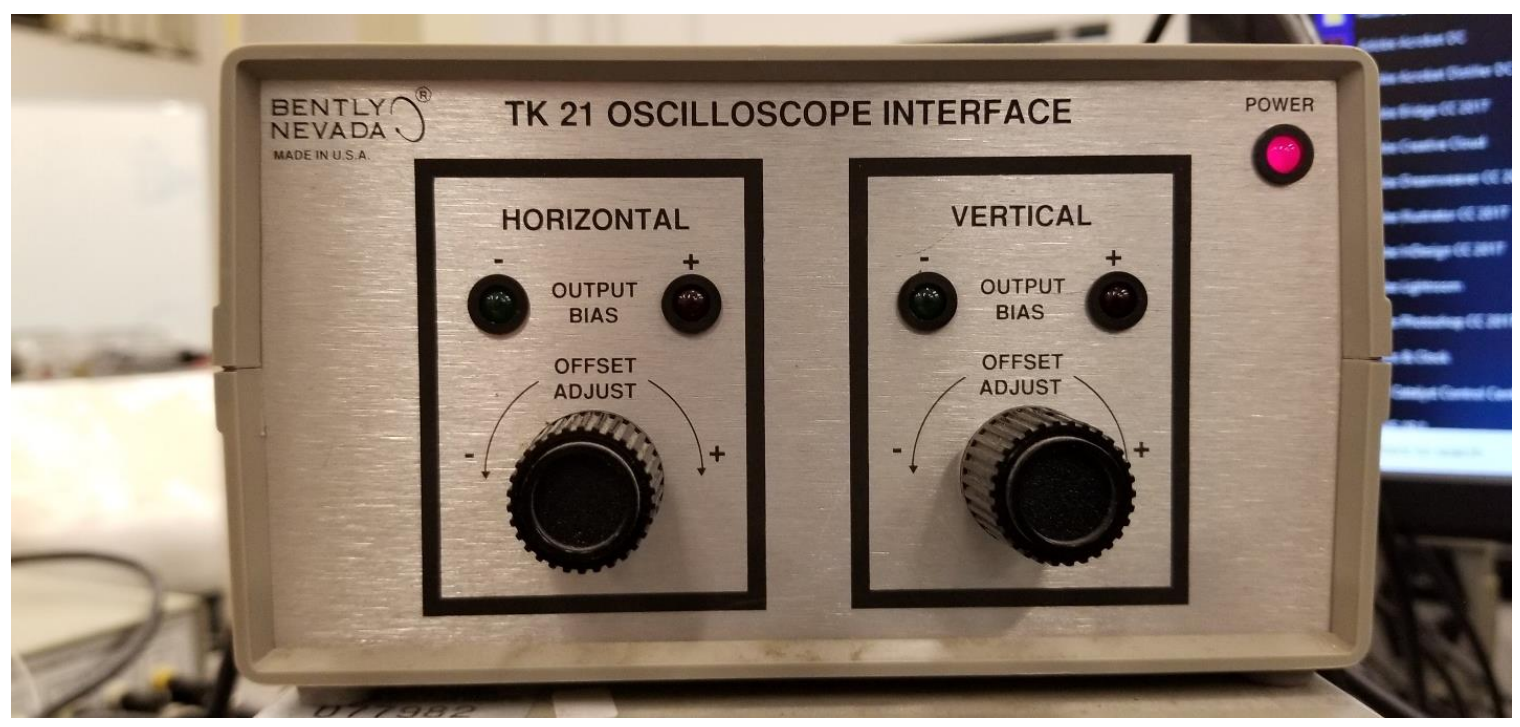

Figure 5. One of the TK21 Oscilloscope Interfaces used with the Rotor Kits in the Cal Poly Vibrations Lab. 


\section{Chapter 3: National Instruments CompactDAQ with MATLAB}

\section{$\underline{3.1 \text { CompactDAQ Overview }}$}

National Instruments CompactDAQ systems are a line of customizable data acquisition systems. A standard CompactDAQ system consists of a chassis that connects to a computer, one or more modules used to collect data, and software to collect this data.

Previous work with the CompactDAQ line of devices made use of the cDAQ ${ }^{\mathrm{TM}}-9174$ chassis coupled with two NI 9215 with BNC modules. This custom assembled data acquisition system, shown in Figure 6, can be used collect data from the RK 4 Rotor Kits in the Cal Poly Vibrations Lab. To do so, a MATLAB app was developed to control this custom CompactDAQ system and process collected data in real time. To simplify naming conventions, this custom system will be referred to as the CompactDAQ for the remainder of this paper.

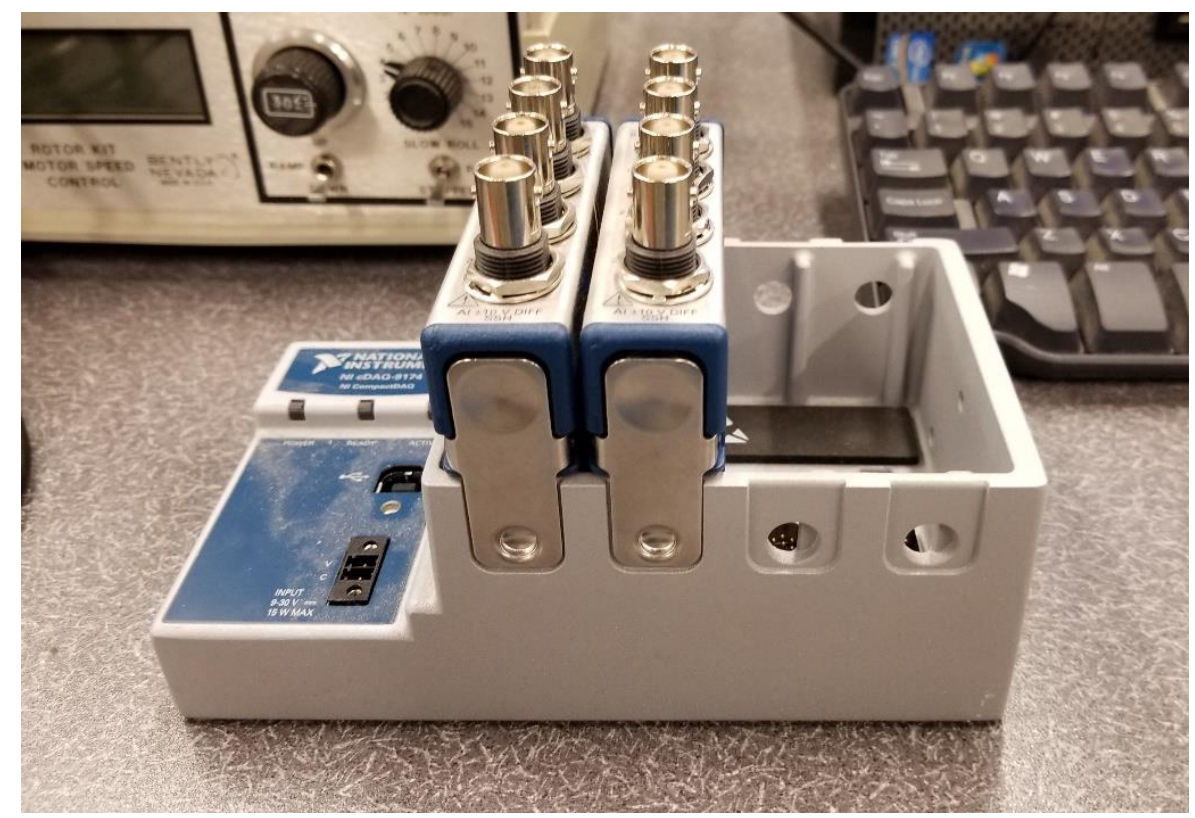

Figure 6. The CompactDAQ system used in the Cal Poly Vibrations Lab. It is assembled from a cDAQ ${ }^{\mathrm{TM}}-9174$ chassis and two NI 9215 with BNC modules. 


\section{2 cDAQTM-9174 Chassis $^{\text {TM }}$}

The cDAQ-9174 is a CompactDAQ chassis with four slots. It connects to a computer using a USB 2.0 high-speed connection and is powered by an external source [6]. As seen in Figure 7, modules can be attached to one of four ports on the back plane of the chassis. The chassis synchronizes attached modules to an internal clock, allowing multiple modules to be used together [7]. It is this capability that allows the cDAQ-9174 to collect data from the Rotor Kit proximity sensors using two NI 9215 modules simultaneously (see sections 2.2 and 3.3).

\subsection{NI 9215 Module}

The NI 9215 is a CompactDAQ module with a 16-bit analog to digital converter. It has four differential analog channels that can be simultaneously sampled. Each channel has a
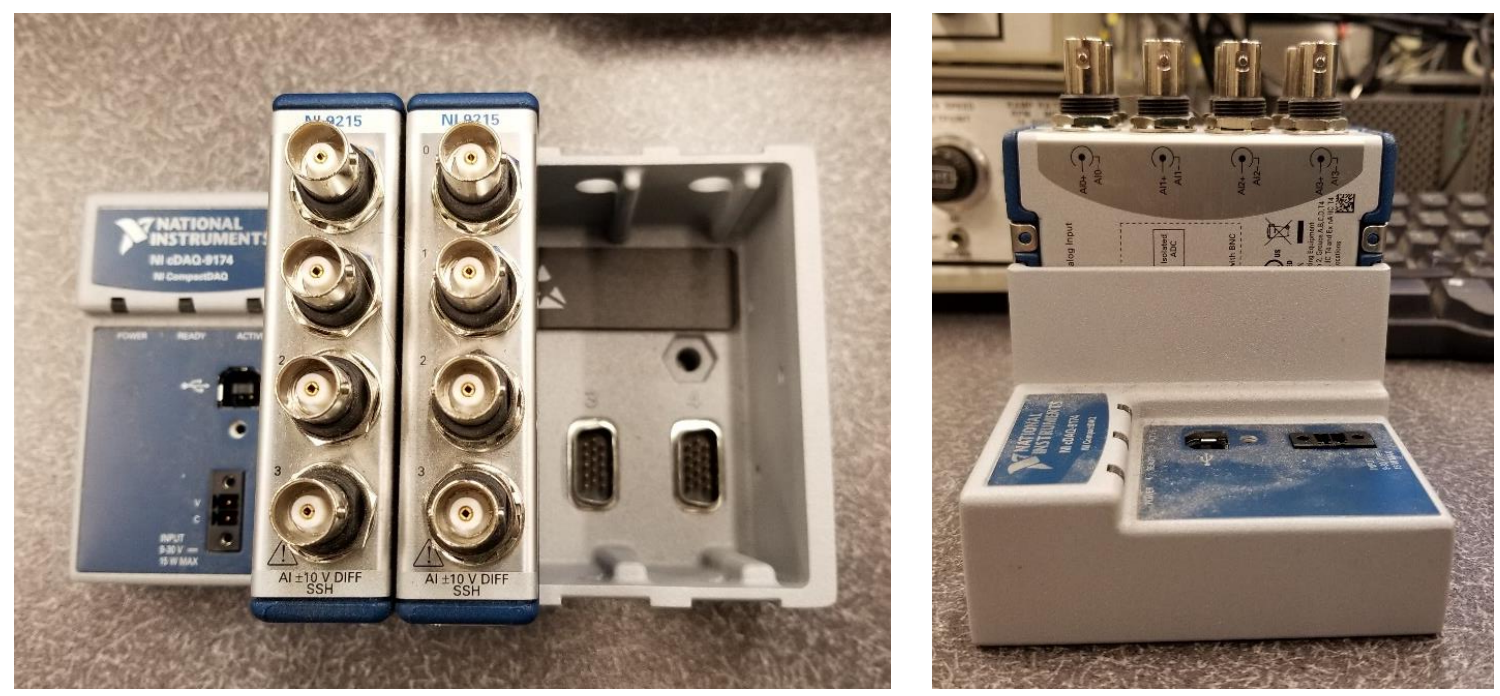

Figure 7. Top (left) and front (right) views of The CompactDAQ system. The two unused module ports can be seen on the left. The side of the NI 9215 modules, shown on the right, includes diagrams showing the connection of the BNC connectors to the ADC differential inputs. 
bipolar range of \pm 10 volts and can be sampled at a rate of up to $100 \mathrm{kSPS}$. The NI 9215 is offered with either BNC, screw terminal, or spring terminal connections [8].

The CompactDAQ used in the Cal Poly Vibrations Lab incorporates two NI 9215 modules with BNC connectors; pictures of these modules with their connectors can be seen in Figure 7. These modules, when paired with the chassis, creates a data acquisition system capable of measuring eight channels simultaneously. When used with the Rotor Kit, up to five of these channels are used (see section 2.2).

\subsection{MATLAB Rotor Kit App}

The MATLAB Rotor Kit App, created by David Baker, is used to collect data from the RK 4 Rotor Kit using the National Instruments CompactDAQ. This app uses the Data Acquisition Toolbox with the Data Acquisition Toolbox Support Package for National Instruments NI-DAQmx Devices add-on to control the CompactDAQ. Data can be collected at a desired sampling rate and optionally plotted in real time [3]. The interface for this app can be seen in Figure 8. 


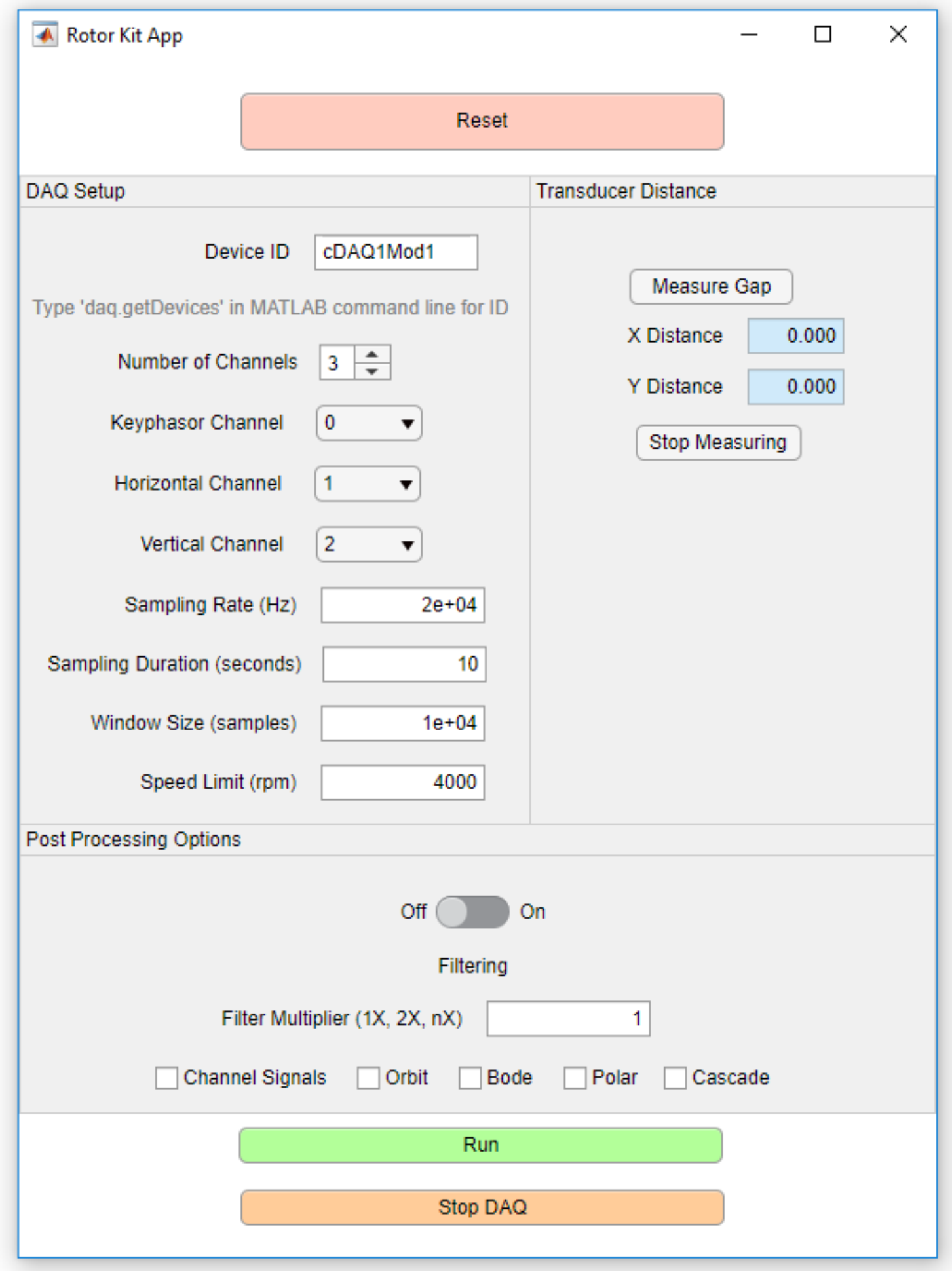

Figure 8. The MATLAB Rotor Kit App interface. Data is collected from the CompactDAQ by entering the desired options and clicking the "Run" button. 


\section{Chapter 4: STM32 USB Data Acquisition System Hardware}

\subsection{Hardware Overview}

The low-cost data acquisition system, seen in Figure 9, was designed around the use of an STMicroelectronics Nucleo development board with an expansion board utilizing an Analog Devices AD760x analog to digital converter. The design of the DAQ allows for any pin compatible and USB capable Nucleo board to be used, though the NucleoF446RE is recommended for its high speed and low cost. The expansion board design provides compatibility for any ADC from the AD760x family of devices, though the AD7609 is recommended as its differential inputs account for DC offset and some noise in a signal. The expansion board additionally allows for configuration of its USB and ADC peripherals. Expansion board design considerations included GPIO configuration, impedance control, analog and digital partitioning, and crosstalk reduction.

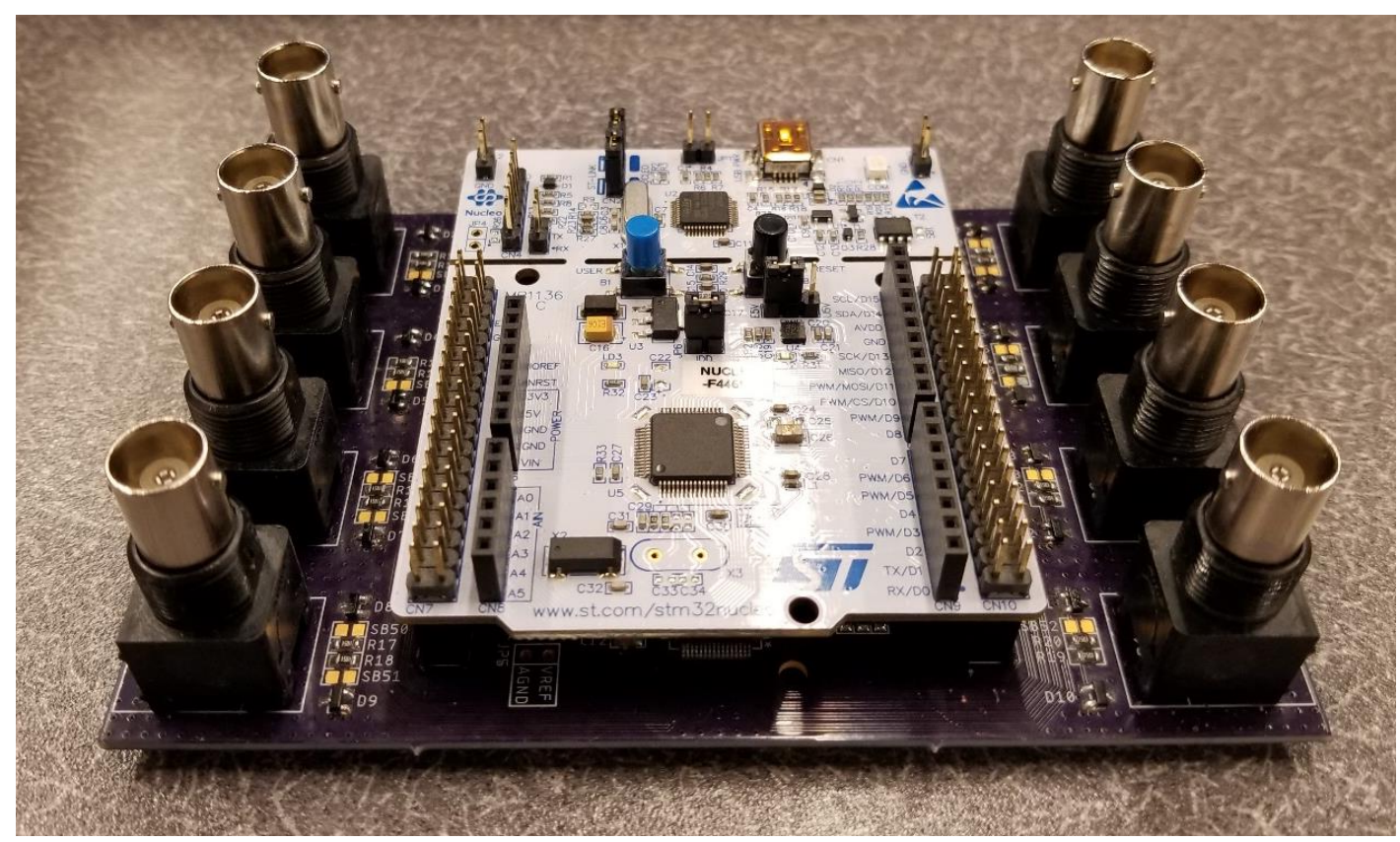

Figure 9. The STM32 USB Data Acquisition System. It is assembled from an STM32 Nucleo development board and a Data Acquisition Expansion Board. 


\subsection{STM32 Nucleo Selection}

This low-cost data acquisition system uses one of the STMicroelectronics STM32 microcontrollers. These 32-bit microcontrollers are based on the ARM Cortex-M family of processors. The Nucleo line of development boards by STMicroelectronics offer many of these microcontrollers in a ready to use format. Three different variations of these development boards exist: Nucleo-32, Nucleo-64, and Nucleo-144. The number associated with each of these variations indicates the number of pins on the STM32 microcontroller. Each variation has different connectors from which the GPIO can be accessed. The Nucleo-32 boards have Arduino Nano compatible connectors, while the Nucleo-64 and Nucleo-144 boards each have Arduino Uno and ST morpho connectors.

When selecting a specific Nucleo board, there were a couple considerations. First, since the design of the DAQ relied on the use of an expansion board, it was desired to maximize compatibility between the number of compatible devices. Since the Nucleo-64 and Nucleo-144 variations shared similar connectors, these boards were considered. The selection was narrowed down to the Nucleo-64 variation, as the smaller size of these boards results in a reduced price. Second, the specific microcontroller on the chosen board was required to support USB connectivity, further narrowing down the number of considered boards. Finally, to maximize the sampling rate of the DAQ, a higher speed device was desired. Of the remaining boards, the best suited board was the NucleoF446RE, a Nucleo-64 development board based on the STM32F446RE microcontroller. This microcontroller included on-chip USB 2.0 full-speed support and could run at a maximum frequency of $180 \mathrm{MHz}$. 


\subsection{Data Acquisition Expansion Board Design}

The Data Acquisition Expansion Board is designed to be used in conjunction with a compatible Nucleo development board. The expansion board incorporates an analog to digital converter to collect data, and a USB 2.0 full-speed port to send data to a computer and optionally power the Nucleo board. The design considerations that went into the expansion board include GPIO configuration, impedance control, analog and digital partitioning, and crosstalk reduction. The front and back sides of the expansion board PCB can be seen in Figure 10 below.
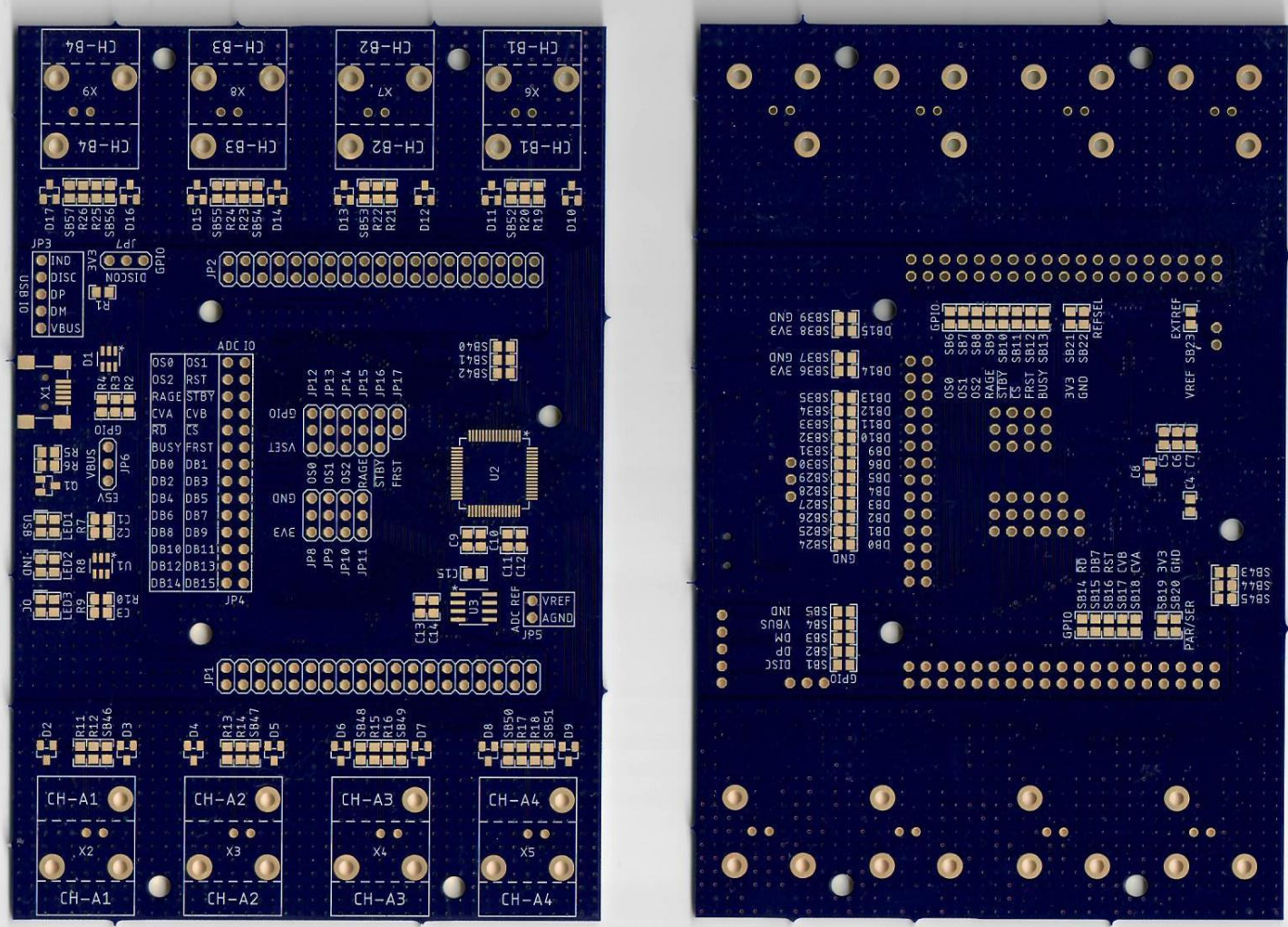

Figure 10. The top (left) and bottom (right) of the Data Acquisition Expansion Board. 


\subsubsection{USB 2.0 Full-Speed Implementation}

The expansion board adds USB 2.0 full-speed device functionality to compatible Nucleo boards. A USB 2.0 full-speed device is theoretically capable of achieving a transfer rate of $12 \mathrm{Mb} / \mathrm{s}$; however, the actual transfer rate typically ranges between $500 \mathrm{~kb} / \mathrm{s}$ and 10 $\mathrm{Mb} / \mathrm{s}$ [9]. This transfer rate makes USB suitable for use in data acquisition systems.

The USB section of the Data Acquisition Expansion Board incorporates a couple different features useful to the operation of the DAQ. These features include three indicator LEDs, two jumpers used to configure the USB connection, and protection circuitry used prevent damage from occurring to both the DAQ and the host computer.

The Data Acquisition Expansion Board includes three USB indicator LEDs. These LEDs are useful for indicating proper operation of the DAQ and diagnosing potential problems. The first LED, colored green, indicates that the DAQ is properly connected to a host computer. This LED only emits light when both the microcontroller and the USB port are receiving power. The second LED, colored yellow, is GPIO driven. Its purpose is user defined; however, it is recommended to use this LED in a manner compliant to the USB Test and Measurement Class specification (see section 5.2.1). The third LED, colored red, indicates that an overcurrent condition is occurring. The operation of this LED is described later in this section.

The power source of the DAQ can be configured using a jumper, allowing the DAQ to act as either a self-powered or a bus-powered device. In self-powered mode, the Nucleo board receives power from an external source; this power can come either from the built in ST-Link debugger and programmer on the Nucleo, or from one of the external supply 
input pins [10]. When using self-powered mode, the USB port power supply line (VBus) is connected to the appropriate GPIO pin on the STM32 microcontroller, allowing the microcontroller to detect when the device is connected to a host computer. In buspowered mode, the Nucleo board receives power through its USB port from the host computer. When using this mode, VBus is disconnected from the microcontroller GPIO; because the device is always connected to the host computer when powered, it is not necessary to detect the connection [11].

Another configuration option of the DAQ is the source pull-up resistor on the USB DP line. Compliant USB full-speed devices are required to pull up the DP line to 3.3 volts using a $1.5 \mathrm{k} \Omega$ resistor. Some STM32 microcontrollers implement the resistor; if using one of the respective Nucleo boards, the extension board pull-up resistor should be disabled. Otherwise, the pull-up resistor is necessary for proper USB operation [11]. The external pull-up resistor is configured using a jumper. To disable the pull-up resistor, the jumper should be removed. However, if the pull-up is necessary, the 3.3 volts source can be selected to either be constant or GPIO controlled. If using a constant 3.3 volts source, the device will always appear connected to the host computer. Alternatively, if the 3.3 volts source is GPIO controlled, the device can either appear connected by driving the pull-up resistor voltage high or appear disconnected by driving the pull-up resistor voltage low (Universal Serial Bus Specification, 2000).

In addition to these configuration features, the Data Acquisition Expansion Board implements some safety features. To protect the DAQ from electrostatic discharge (ESD) from the USB port, a USBLC6-2 is used. The USBLC6-2 is an ESD protection IC that is USB 2.0 compliant and can maintain signal integrity on the data lines due to its 
low capacitance [12]. In addition to ESD protection, the VBus detection GPIO is protected using a voltage divider. When in self-powered mode, if the DAQ is plugged into a host computer before receiving power, a voltage will be applied to the VBus detection GPIO on the unpowered microcontroller. On an unpowered microcontroller, this GPIO can withstand a maximum of 4 volts [11]. The voltage divider between the USB VBus line and the VBus detection GPIO drops the line 5 volts down to approximately 3.3 volts, well below the specified 4 volts maximum. The final means of protection is the use of the AP2552 current limited switch. When using the DAQ in buspowered mode, the AP2552 prevents the DAQ from pulling more than $500 \mathrm{~mA}$ from the host computer. During an overcurrent condition, the active-low open drain fault pin is driven low [12]. The red indicator LED is connected between the power line and the fault pin and indicates when this condition occurs.

\subsubsection{Analog to Digital Converter Implementation}

The expansion board implements an analog to digital converter (ADC) from the Analog Device AD760x family of devices. These multi-channel ADCs have simultaneously sampled bipolar analog inputs capable of measuring analog inputs of up to \pm 10 volts, matching the maximum measurable input voltage of the CompactDAQ NI 9215 module. These devices have a maximum sampling rate on the order of $100 \mathrm{kSPS}$, making them suitable for high speed data acquisition. The design of the Data Acquisition Expansion Board supports implementation of any one of these devices; differences between the devices are handled by using solder bridges. However, to account for DC offset, the AD7609 is the best suited ADC for use with Data Acquisition Expansion Board. A comparison between the ADCs in the AD760x family can be seen in Table 2 . 
Table 2. A comparison between the Analog Devices AD760x family of ADCs.

\begin{tabular}{|l|c|c|c|c|c|l|}
\hline $\begin{array}{c}\text { Part } \\
\text { Number }\end{array}$ & $\begin{array}{c}\text { Resolution } \\
\text { (bits) }\end{array}$ & $\begin{array}{c}\text { Sample } \\
\text { Rate } \\
\text { (kSPS) }\end{array}$ & Channels & $\begin{array}{c}\text { Over- } \\
\text { sampling }\end{array}$ & $\begin{array}{c}\text { Analog } \\
\text { Input Type }\end{array}$ & Interface \\
\hline AD7605-4 & 16 & 300 & 4 & No & $\begin{array}{l}\text { Single } \\
\text { Ended }\end{array}$ & $\begin{array}{l}\text { Serial, } \\
\text { Parallel } \\
\text { Byte, } \\
\text { Parallel }\end{array}$ \\
\hline AD7606-4 & 16 & 200 & 4 & Yes & $\begin{array}{l}\text { Single } \\
\text { Ended }\end{array}$ & $\begin{array}{l}\text { Serial, } \\
\text { Parallel } \\
\text { Byte, } \\
\text { Parallel }\end{array}$ \\
\hline AD7606-6 & 16 & 200 & 6 & Yes & $\begin{array}{l}\text { Single } \\
\text { Ended }\end{array}$ & $\begin{array}{l}\text { Serial, } \\
\text { Parallel } \\
\text { Byte, } \\
\text { Parallel }\end{array}$ \\
\hline AD7606 & 16 & 200 & 8 & Yes & $\begin{array}{l}\text { Single } \\
\text { Ended }\end{array}$ & $\begin{array}{l}\text { Serial, } \\
\text { Parallel } \\
\text { Byte, } \\
\text { Parallel }\end{array}$ \\
\hline AD7608 & 18 & 200 & 8 & Yes & $\begin{array}{l}\text { Single } \\
\text { Ended }\end{array}$ & $\begin{array}{l}\text { Serial, } \\
\text { Parallel } \\
\text { Byte, } \\
\text { Parallel }\end{array}$ \\
\hline AD7607 & 14 & 200 & 8 & Yes & $\begin{array}{l}\text { Single } \\
\text { Ended }\end{array}$ \\
\hline & 18 & 200 & 8 & Yes & Differential & $\begin{array}{l}\text { Serial, } \\
\text { Parallel }\end{array}$ \\
\hline
\end{tabular}

The analog inputs to the AD760x are interfaced using BNC connectors. These are the same connectors commonly used with oscilloscopes as well as the NI 9215 with BNC modules used with the CompactDAQ. The AD760x analog inputs can withstand overvoltage conditions up to \pm 16.5 volts. However, to match the \pm 30 volts overvoltage protection provided by the NI 9215 module, each analog input incorporates two back-toback Zener diodes and a resistor (see Appendix A). The selected Zener diodes have a nominal Zener voltage of 15 volts; this Zener voltage was chosen as it clamps the analog inputs to a magnitude voltage lower than the maximum allowed by the AD760x while 
avoiding any significant clipping from occurring at the maximum measurable magnitude of 10 volts. The selected resistor has a resistance of $1.5 \mathrm{k} \Omega$, which, given the maximum design magnitude 30 volts at the $\mathrm{BNC}$ and a clamping voltage of 15 volts, limits the maximum total current to $10 \mathrm{~mA}$. Even if most of the current passes through the Zener diodes, the Zener diodes stay within a safe operating range.

The Data Acquisition Expansion Board is designed to allow for quick configuration of the optional inputs and outputs of the AD760x using jumpers. The optional inputs include the three oversampling pins (only applicable for supported devices), the range pin, and the standby pin. The one optional output is the first data pin. The use of these pins is described in the datasheet for the applicable device. The five inputs can either be controlled by GPIO or set to a constant logic level. If configuring the oversampling and range inputs to use a set logic level, the logic level can be set high (3.3 volts) or low (ground) using a second set of jumpers. Conversely, configuring the standby pin to use a set logic level always applies a high logic level to the pin; a constant low logic level would put the AD760x either in standby or shutdown mode, and is therefore not useful. Unlike the inputs, the jumper for the one output pin is optional. Adding the jumper connects the first data pin to the microcontroller GPIO, while removing it disconnects the pin from the GPIO.

The AD760x can further be configured by adding or removing various solder bridges. The Data Acquisition Expansion Board is designed to interface with the AD760x using a serial connection. However, modifying the appropriate solder bridges allows the ADC to be interfaced in parallel or (if supported) parallel byte mode. If using either of these modes, the AD760x digital IO breakout must be used. Additionally, the AD760x can be 
configured to use either its internal reference or an external reference. The external reference can be provided by the optional on-board ADR 421 voltage reference or can be provided off-board using the analog reference breakout for the AD760x. Modifying the appropriate solder bridges allows for one of these voltage reference sources to be selected. Appendix $\mathrm{C}$ details how to make these modifications.

\subsubsection{GPIO Configuration}

The Data Acquisition Expansion Board is designed to attach directly to the ST Morpho connectors on the STM32 Nucleo development board. The Nucleo boards are designed to be compatible with many of the STM32 microcontrollers, and therefore provide similarity in the ST Morpho connector layout across different Nucleo boards. However, GPIO differences between different microcontrollers results in some ST Morpho connector pins being applicable to only some Nucleo boards. Additionally, some GPIO are disconnected from the ST Morpho connectors by default for other uses by the board, while others are routed to different connector pins depending on the microcontroller used. To maximize compatibility between boards, GPIO that were always available on the same connector pins across multiple Nucleo boards were selected. The only exception to this is the available SPI GPIO, which differ between different microcontrollers; however, the design of the Nucleo boards ensures that an SPI connection is always available on the same ST Morpho pins, regardless of the microcontroller [10, 13].

In addition to the ST Morpho connection, the Data Acquisition Expansion Board provides means for a custom connection to the Nucleo board. This ability to create a custom connection allows for prototyping of different configurations; for example, the 
AD760x can be interfaced using a parallel connection instead of the default serial connection. Configuration of the expansion board is achieved using solder bridges and pin breakouts. To modify an input or output connection, the appropriate solder bridge must be removed, and a wire run from the breakout of the respective input or output pin to the ST Morpho connector. Modifications to optional pins on the AD760x maintains compatibility with the quick configuration design described in section 4.2.2. Details on input and output modifications can be found in Appendix C.

\subsubsection{Board Design Considerations}

To ensure proper operation of Data Acquisition Expansion Board, some design considerations needed to be taken. These considerations included trace impedance control, mixed signals partitioning, and electromagnetic interference reduction.

One consideration while designing the Data Acquisition Expansion Board was trace impedance control. Many of the traces on the Data Acquisition Expansion Board do not require a specific impedance. However, to maintain compliance, the two USB 2.0 data lines require a differential impedance of $90 \Omega \pm 15 \%$ [9]. To determine the data line trace characteristics necessary for impedance control, the Edge Coupled Microstrip Impedance Calculator from EEWeb.com was used. The substrate trace thickness, substrate height, and substrate dielectric constant were taken from the OSH Park 4-layer board specification. The trace spacing was chosen to be 6 mils (chosen to allow compatibility with the OSH Park 2-layer board specification). Using these specifications with the EEWeb.com calculator, and rounding to the nearest $0.005 \mathrm{~mm}$, a required trace width of $0.375 \mathrm{~mm}$ was found (see Figure 11). Using this trace width with worst case 
manufacturing tolerances, the differential impedance range is $85.5 \Omega$ to $93.8 \Omega$, well within the $90 \Omega \pm 15 \%$ specification.

Another consideration was partitioning the Data Acquisition Expansion Board. To preserve the quality of analog signals sampled, it is necessary to minimize the noise in the area surrounding the analog traces. Digital signals, particularly high-speed signals, are a

\section{EDGE COUPLED MICROSTRIP IMPEDANCE CALCULATOR}

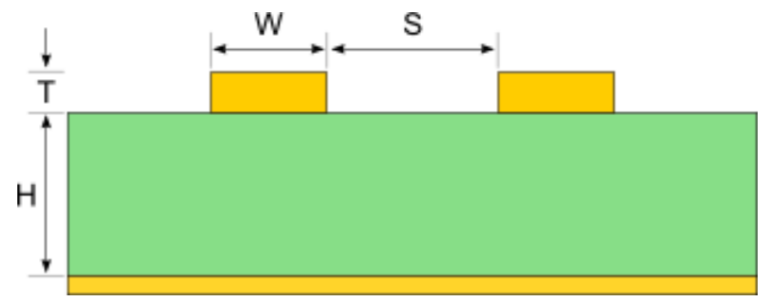

\section{INPUTS}

Trace Thickness

Substrate Height

Trace Width

Trace Spacing

Substrate Dielectric
$\mathbf{T}$

H1

W

S

Er

\section{4}

6.7

.375

6

3.66

\section{OUTPUTS}

Odd (Z): 45.0 ohms

Even (Z): 48.7 ohms

Common (Z): 24.4 ohms

Differential (Z): 89.9 ohms

Figure 11. Calculation of the required USB data line trace width using the Edge Coupled Microstrip Impedance Calculator from EEWeb.com. 
source of noise, and can cause coupling in nearby traces (see crosstalk discussion below). Isolating this digital noise from the analog signals can be achieved by partitioning the board into analog and digital sections. This partitioning is achieved by running only analog traces through the analog section and by splitting the ground plane into analog and digital sections. Doing so keeps digital currents out of the analog sections of the board, and therefore prevents coupling between the digital and analog signals. To provide a return path for analog currents, the analog and digital grounds meet at one spot; in the case of the Data Acquisition Expansion Board, the two grounds meet at the ADC; the ground plane split and connection can be seen in Appendix B. It is important that traces do not cross ground plane split; since the ground signal cannot cross the split, it must flow around the split, increasing the signal loop area which in turn increases electromagnetic interference $[14,15]$.

The final design consideration involved reducing electromagnetic interference (EMI). In high speed designs, a major contributor to EMI is crosstalk, the capacitive and inductive coupling between two traces. Crosstalk occurs when a non-constant signal, such as a pulse, travels through one trace (the driven trace) and causes noise in a neighboring trace (the victim trace). There are two types of crosstalk: forward and backward. Forward crosstalk increases with the length of the coupled traces; however, the capacitive and inductive coupling cancel in homogenous environments, making forward crosstalk negligible. Conversely, backwards crosstalk increases over the length of coupled traces to a maximum magnitude, but then does not increase any further. Unlike forward crosstalk, the capacitive and inductive coupling add, and therefore cannot be neglected. 
Crosstalk increases as the frequency of a signal increases and the distance between traces decreases [16].

Since it is not feasible to reduce the signal frequency in high speed applications, traces must be spaced to reduce crosstalk. Many different crosstalk calculators and simulators exist and are useful for designs that require crosstalk reduction. For the design of the expansion board, the Microstrip Crosstalk Calculator from EEWeb.com was used.

The spacing between traces was calculated by assuming worst case conditions. Using the STM32F446 and the AD7609 datasheets, the highest possible low-level voltage on an output pin is 0.59 volts lower than the low-level threshold; similarly, lowest possible high-level voltage on an output pin is 0.59 volts higher than the high-level threshold. Therefore, to avoid crossing the logic level threshold, the maximum allowable crosstalk between traces is 0.59 volts. To account for other possible sources of noise, a factor of safety of 3 was used, bringing the maximum allowable crosstalk down to approximately 0.196 volts. Additionally, the fastest rise time was taken from the STM32 datasheet, and the dielectric constant and maximum possible substrate height from the OSH Park 4-layer board specification. The trace length was conservatively set to 10 inches as it exceeds that of any of the traces on the Data Acquisition Expansion Board. Inputting this data into Microstrip Crosstalk Calculator from EEWeb.com, and rounding to the nearest 0.01 $\mathrm{mm}$, the minimum allowable trace spacing was found to be $0.75 \mathrm{~mm}$ (see Figure 12). 


\section{MICROSTRIP CROSSTALK CALCULATOR}

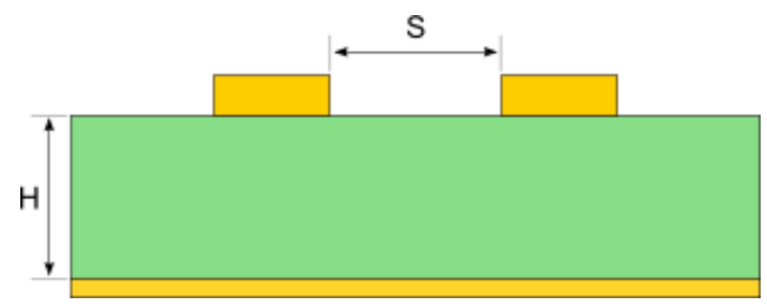

\section{INPUTS}

\begin{tabular}{|c|c|c|c|}
\hline Source Rise Time & Tr & 2.5 & ns \\
\hline Source Voltage & $\mathbf{V}$ & 3.3 & Volts \\
\hline Length of Parallel Routes & $\mathbf{L}$ & 10 & inch $V$ \\
\hline Substrate Height & $\mathbf{H}$ & 7.37 & mil $\vee$ \\
\hline Trace Spacing & $\mathbf{S}$ & 0.75 & $\mathrm{~mm} \vee$ \\
\hline Substrate Dielectric & Er & 3.66 & \\
\hline
\end{tabular}

\section{OUTPUT}

Cross Talk Coeff: $-24.6 \mathrm{~dB}$

Coupled Voltage: 0.194 Volts

Figure 12. Calculation of the trace spacing necessary to limit crosstalk using the Microstrip Crosstalk Calculator from EEWeb.com.

\subsection{Power Supply Considerations}

Some consideration needs to be taken when powering the STM32 USB Data Acquisition System to ensure proper operation. The STM32 Nucleo can be powered by four sources:

1. The on board STLink USB connection, which provides a nominal 5 volts. 
2. An external 5 volts source into the E5V pin; in the case of the DAQ, this can be configured to be the USB 2.0 full-speed port.

3. An external 3.3 volts source into a 3.3 volts pin.

4. An external 7 volts to 12 volts source into the VIN pin. This voltage is stepped down to a nominal 5 volts through the on-board linear regulator.

Each of these four sources of power allow the STM32 microcontroller to operate normally [10]. However, not all configurations can be used with the expansion board due to the power requirements of the AD760x analog to digital converter. To ensure proper operation, the AD760x requires $5.00 \pm 0.25$ volts [17]. As such, an external 3.3 volts power source cannot be used. Additionally, if using either the STLink USB connection or the expansion board USB connection, only a high-powered hub must be connected to. High-powered USB hubs provide between 4.75 volts and 5.25 volts, the same range as is required by the AD760x. However, low-powered hubs provide between 4.4 volts and 5.25 volts. Although it is possible for the AD760x to operate properly if connected to a low-powered hub, the design of the expansion board does not ensure it. 


\section{Chapter 5: STM32 USB Data Acquisition System Software}

\subsection{Software Overview}

Code was written for the STM32 USB Data Acquisition System to allow it to simultaneously collect data using an analog to digital converter and transfer this data to a host computer. This multitasking is achieved using FreeRTOS with two tasks: a USB task and an analog to digital converter task. Each of these tasks implement an STM32 compatible library for their respective peripheral: the USB task uses the USB Test and Measurements Class device library, while the ADC task uses the AD760x ADC library.

Additionally, code was written for the host computer to read and process the collected data. A console application is used to read DAQ data into a comma-separated values (CSV) file. This application uses the USB core host library to select a USBTMC device, and the USB Test and Measurements Class host library to read data from the device. MATLAB is then used to process and plot the data read from the DAQ.

\subsection{STM32 Peripheral Libraries}

The STM32 USB Data Acquisition System uses two main peripherals: the USB Test and Measurement Class device interface, and the AD760x analog to digital converter. In order to control these peripherals, libraries were developed for each of these peripherals for use with the STM32 line of microcontrollers. 


\subsubsection{USB Test and Measurement Class Device Library}

The DAQ is designed to connect to a host computer using a USB 2.0 full-speed connection (see section 4.3.1). USB devices have one or more configurations that can be selected by a host computer. Each configuration has one or more interfaces, each of which provide a specific functionality for the device. A device interface specifies a class, subclass, and protocol code, which allows the host computer to load an appropriate driver for the device $[9,18]$. The USB Test and Measurement Class specification provides a standard means for test and measurement devices to collect and send data to a host computer [19].

The USB Test and Measurement Class device library allows STM32 microcontrollers to both act as USB test and measurement devices and be recognized by host computers as such. This library is designed to work with one of two available USB libraries provided by STMicroelectronics. One of these libraries is a part of the Standard Peripheral Library (SPL), and the other is a part of the Hardware Abstraction Layer (HAL) library. The SPL version has less overhead code and is therefore more streamlined than the HAL; however, as the SPL version is older and no longer supported, it lacks some newer features available to the HAL library, such as Link Power Management. The USBTMC class code interfaces with one of these libraries using an adapter code file, allowing for either one to be used. For the purpose of the DAQ, the SPL library was selected because of its efficiency and because the extra functionality provided HAL library is not needed. The API for the USBTMC device library can be found in Appendix D. 


\subsubsection{AD760x Analog to Digital Converter Library}

The DAQ implements an analog to digital converter from the AD760x family of devices. These ADCs have more than one means to interface with a microcontroller (see section 4.3.2). As such, it was necessary to create a library that could interface with any AD760x device while maintaining a universal format. This was achieved using two C++ classes: a core class and a board support package (BSP) class. The BSP class provides all hardware control for the AD760x device, including setup and sample collection. This class uses function pointers to allow users to implement their specific hardware setup. The core class provides a common interface for controlling the ADC. Its methods control when and how to use the user defined BSP functions to ensure proper operation of the ADC. This class also provides post processing of samples, such as setting sample justification, endianness, and byte count. The API for both AD760x library classes can be found in Appendix D.

\subsection{Data Acquisition System RTOS and Finite State Machines}

It is often necessary for a microcontroller to control multiple peripherals simultaneously. Each of these peripherals have a finite number of states in which they can be. A finite state machine is a model that encompasses each of these states, the operations they perform, and the conditions that cause a change of state. Each finite state machine can be implemented as a task for a microcontroller to perform. Multitasking is achieved when multiple tasks are operating simultaneously, and results in a complete system capable of performing a job. A real time operating system (often abbreviated as RTOS) can be used 
to achieve multitasking. Using this system, each task is assigned a priority and a run rate, allowing the RTOS to determine when and how often to run a task [20].

The STM32 USB Data Acquisition System implements an RTOS using a C++ wrapped implementation of FreeRTOS. Two tasks are used: a USB task and an analog to digital converter task. The USB task uses the USBTMC library to receive and parse commands sent from a host computer, as well as send requested data. The ADC task uses the AD760x library to collect data samples at a requested sample rate. The two tasks communicate using shared device flags, command variables and flags, and pointers to data buffers. The task diagram as well as the state diagrams for both tasks can be seen in Appendix G.

\subsubsection{USB Task}

The USB task handles communication with the host computer. This task uses the USBTMC library to present itself as a test and measurement device, and both parses received commands and sends requested data to the host computer. This task has three states:

State 0: Initialize the USB task. This state handles initialization of the USB peripheral using the USBTMC library, allocates the buffers used for data collection and transfers, and sets up the timer used for state 2 . This state also tries to obtain device information from the ADC task, and transitions to state 1 once the information is retrieved. To avoid errors, the USB, buffer, and timer setup code only executes the first time this state runs. 
State 1: Reset the USB task. This state resets all the USB task variables and the inter-task command flags to their default values and checks if the USB peripheral has been configured by a host computer. This state enables the USB task timer and then transitions to state 2 once all device flags have been cleared and the USB peripheral configured. To avoid errors, this state only resets the USB task if the respective reset flag is set; this flag is cleared after performing the reset.

State 2: Receive and parse DAQ commands. This state handles all USB transfers between the device and host, and parses all commands received by the host. Due to the high speed nature of this state, it is handled by a timer interrupt that executes once every 50 microseconds. This state is split into 5 substates that each handle a different part of the USB transfer and command parsing process. If one of its substates disables the USB task timer due to a reset command or the device being disconnected from the host, the USB task transitions to state 1 .

State 2 encompasses all USB transfer and command parsing operations. Maintaining the finite state machine model, the operations executed by this interrupt are all contained within state 2 of the USB task, and are split into 5 substates:

Substate 0: Submit command transfer. This substate handles submission of the transfer used to receive commands to the USB peripheral. State 2 transitions to substate 1 once the transfer has been successfully submitted 
or disables the timer if any of the device flags have been set or the device has been disconnected from the host.

Substate 1: Check if command received. This substate checks if a command has been received from the host computer. If a command that does not expect a response is received, state 2 transitions to substate 2 .

Alternatively, if a command expecting a response is received, state 2 transitions to substate 3 . If any of the device flags are set or the device has been disconnected from the host, this substate disables the timer and transitions to substate 0 , regardless of whether a command has been received.

Substate 2: Parse device commands. This substate handles the parsing of commands received by the host computer. Multiple commands may be received in one transfer; however, to minimize the execution time of the timer interrupt, only one command is parsed every time the interrupt executes; a list of the defined commands can be seen in Table 3 below. After

Table 3. A description of all commands used by the STM32 USB DAQ.

\begin{tabular}{|l|c|c|l|}
\hline \multicolumn{1}{|c|}{ Command Type } & $\begin{array}{c}\text { Length } \\
\text { (Bytes) }\end{array}$ & $\begin{array}{c}\text { Command } \\
\text { Character }\end{array}$ & \multicolumn{1}{c|}{ Data } \\
\hline Device Info Request & 1 & 'I' & None \\
\hline Set Oversampling Ratio & 2 & 'O' & Oversampling Bit Code \\
\hline Set Voltage Range & 2 & 'R' & Voltage Range Flag \\
\hline Enable/Disable Channels & 2 & 'C' & Channel Bitmask \\
\hline Set Sample Justification & 2 & 'J' & Sample Justification Flag \\
\hline Set Raw Data Format & 2 & 'F' & Raw Data Format Bitmask \\
\hline Begin Data Collection & 5 & 'B' & Sampling Rate \\
\hline End Data Collection & 2 & 'E' & Reset Flag \\
\hline End of Message & 1 & ' $\mathrm{n}$ ' & None \\
\hline
\end{tabular}


parsing of the commands has completed, if the host has sent an end of message (EOM) flag, the appropriate inter-task command variables and their respective flags are updated. If the host has not sent the EOM flag, the updating of the inter-task command variables and flags is deferred until the EOM flag has been received. Once all commands have been parsed, state 2 transitions to substate 0 ; additionally, if a device reset command has been received, the task reset flags are set and the timer is disabled. If any of the device flags have been set or the device has been disconnected from the host computer, the task disables the timer and transitions to substate 0 regardless of whether all commands have been parsed.

Substate 3: Submit response transfer. This substate handles the submission of the transfer used to send requested data to the host computer. If the requested data is available, this substate sets up the transfer buffer, submits the transfer, and transitions to substate 4 . If any of the device flags have been set or the device has been disconnected from the host, this substate disables the timer and transitions to substate 0 , regardless of whether the transfer has been submitted.

Substate 4: Check if response sent. This substate checks if the response transfer has been sent to the host computer. After the transfer has completed, if an inter-task ADC data buffer was used for the transfer, the buffer index is updated. Additionally, if the ADC data buffer is empty, its write flag is set, allowing the ADC task to store new data in the buffer. State 2 
transitions to substate 0 once the transfer has completed. If any of the device flags have been set or the device has been disconnected from the host, this substate disables the timer and transitions to substate 0 , regardless of whether the transfer has been completed.

The USB task was given the same priority as the ADC task, since neither is more important than the other; both are required to run at regular intervals to ensure proper operation of the DAQ. This task is configured to run at two different rates: the state level operations are run once every 100 milliseconds, while the state 2 substate operations run every 50 microseconds. This large discrepancy is due to the different timing requirements for different parts of the task. The state level operations do not need to be executed as often, and do not affect the speed and efficiency of the DAQ since they only handle task initialization and device reset operations; therefore, the task is configured to run once every 100 milliseconds to minimize the amount of time the microcontroller spends executing these operations. Conversely, the rate at which the USB transfer and command operations are performed are crucial to the efficiency of the DAQ. As such, these operations, handled by the state 2 substates, are configured to run once every 50 microseconds. This run frequency was chosen as it allows the longest practical command to be received between runs while maintaining a high parse rate of these commands.

The FreeRTOS kernel operates at a tick rate of 1 millisecond by default. It is possible to increase this operation speed, allowing the state 2 substates to be run using the FreeRTOS task scheduler; however, doing so would decrease the efficiency of the DAQ as the microcontroller would spend more time executing the FreeRTOS kernel code. As such, 
the FreeRTOS task scheduler runs the less frequent state level operations while a timer interrupt runs the high frequency substate level operations.

\subsubsection{Analog to Digital Converter Task}

The analog to digital converter task handles analog data collection. This task uses the AD760x library in conjunction with the USB DAQ board support package to handle configuration of the ADC as well as data collection at a specified frequency. This task has four states:

State 0: Initialize the ADC task. This state handles initialization of the AD760x device using the USB DAQ board support package, sets up the timer used for state 3 , and sets the device information used by the USB task. This state also tries to obtain the data buffers used for data collection and transfer, and transitions the ADC task to state 1 once these buffers are retrieved. To avoid errors, the ADC, timer, and information code only executes the first time this state runs.

State 1: Reset the ADC task. This state resets all the ADC task variables, as well as the AD760x hardware and default configurations. The ADC task transitions to state 2 once all device flags have been cleared. To avoid errors, this state only resets the ADC task if the respective reset flag is set; this flag is cleared after performing the task reset.

State 2: Configure the ADC. This state checks if ADC commands have been parsed by the USB task. If any inter-task ADC configuration command flags have 
been set, the AD760x is configured as appropriate. If the inter-task data collection flag is set, this state configures the ADC task timer period to match the requested data collection rate, enables the ADC task timer, and transitions to state 3. If any of the device flags have been set, the ADC task transitions to state 1 , regardless of whether any ADC commands have been parsed.

State 3: Collect analog data. This state handles collection of analog data using the AD760x. Due to the time accuracy needed, data collection is handled by a timer interrupt that runs at the data collection rate set in state 2 . If the interrupt disables the timer due to any of the device flags being set, the ADC task transitions to state 1 . Alternatively, if the interrupt disables the timer due to the inter-task data collection flag being cleared, the ADC task transitions to state 2 .

The operations performed in state 3 are handled by a timer interrupt to ensure that data samples are collected at a regular rate. Unlike state 2 in the USB task, all operations are handled at once, and thus are not split into substates. The interrupt executes as follows:

Interrupt: Each time the interrupt runs, it first checks whether any of the device flags have been set. If any have, the interrupt disables the timer and exits without executing any of the remaining operations. If all device flags are cleared, the interrupt then checks whether the inter-task data collection flag is set. If the flag is cleared, the interrupt disables the timer, but does not exit. Next, if the current buffer is full or the data collection flag is 
cleared, the interrupt clears the buffer write flag, sends the current buffer index to the USB task for transfer, and sets the index for the next buffer to be filled. Finally, if the data collection flag is still set, the interrupt initiates sampling for the next data point if the write flag of the current (or new) buffer is set and increments the data point counter regardless of whether sampling was initiated.

The ADC task was given the same priority as the USB task, since neither is more important than the other; both are required to run at regular intervals to ensure proper operation of the DAQ. This task is configured to run once every 10 milliseconds; however, data is collected at the frequency requested by the host computer. The task initialization, reset, and configuration operations are not critical to the efficiency of the DAQ, and do not need to run often; however, since configuration of the ADC occurs every time a new set of data is collected, the ADC task should run at a faster rate than the USB task, which only handles task initialization and reset on at the state level (see section 5.3.1). Therefore, a run rate 10 times faster than that of the USB task was chosen, allowing the task to run more often while keeping the amount of time the microcontroller spends executing these operations low. The FreeRTOS task scheduler is used to run the task initialization, reset, and configuration operations, while a timer interrupt is used to run the data collection operation.

\section{$\underline{5.4 \text { Host Computer USB Libraries }}$}

When connecting and communicating with USB device, the host is responsible for managing many aspects of the connection. This includes enumerating and configuring 
the device and handling data transfers, among other things [9]. LibUSB, an open sourced cross-platform $\mathrm{C}$ based library, handles the low-level aspects of these operations, making it easy to write portable USB code. Two USB libraries were created using LibUSB: the USB core host library, and the USB Test and Measurement Class host library.

\subsubsection{USB Core Host Library}

The USB core host library provides two main areas of functionality. Its first area of functionality is acting as a wrapper library for the LibUSB library. This library makes standard USB operations such as retrieving device information and sending USB control requests easier by providing an abstraction layer over the LibUSB code. This abstraction layer allows these operations to be performed without needing to know too much about the underlying USB operations. The second area of functionality is the creation and handling of a USB class device list. This list contains all devices with interfaces adhering to a specific USB class code and specifies the specific configurations and interfaces that adhere to this class code. The device list can additionally be limited to a specified set of vendors and products. This list is useful in situations where it is required that a user select a specific device from a list of compatible devices; for example, selecting a specific data acquisition system to collect data from (see section 5.5.1). The API for this library can be found in Appendix E.

\subsubsection{USB Test and Measurement Class Host Library}

The USB Test and Measurement Class specification provides a standard means for a host computer to communicate with and collect data from test and measurement devices [19]. The USB Test and Measurement Class host library provides a cross-platform 
implementation of this specification using the LibUSB and USB core host libraries. To maintain consistency with the STM32 USBTMC device library, the USBTMC host library code is written in C. This library handles all USBTMC host operations, including initializing and configuring the USBTMC device as well as sending and receiving data, among other things. The API for the USBTMC host library can be found in Appendix E.

\subsection{Rotordynamic Data Acquisition and Processing}

Reading and processing rotordynamic data from the STM32 USB Data Acquisition System is performed in two steps. Communication with the DAQ is achieved using a console application. This application retrieves data from the DAQ using user specified operating conditions and saves this data to a comma-separated values (CSV) file. This

data is then read into MATLAB and processed using the same methods as the MATLAB app written for the National Instruments CompactDAQ [3].

\subsubsection{Data Acquisition Console Application}

Rotordynamic data is retrieved from the DAQ using the Data Acquisition Console Application. This application uses the USB core host library to select a DAQ, and the USB Test and Measurement Class host library communicate with and read data from the selected DAQ. The read data is stored in a comma-separated values (CSV) file to be used for processing an analysis. Unlike the RTOS and finite state machine model used with the STM32 microcontroller, the data acquisition program is very linear; this program does not need to perform extensive multitasking since it is only collecting data and saving it to a CSV file. The program flow can be split into three stages: 
Stage 1: Device selection. This stage of the program handles the selection of the device and its configuration and interface to use. The host computer finds all attached devices with a USBTMC interface and creates a numbered list for a user to choose from. Once the user has selected a device, the applicable device configurations and USBTMC interfaces are listed. The user then selects the desired configuration and interface to be used.

Stage 2: DAQ initialization and setup. This stage of the program handles initialization of the DAQ and sets its desired operating settings. This stage first initializes the DAQ and then sends a reset command to ensure that the DAQ is in its default state. Next, device information is requested and obtained. Based on the information obtained from the device, the user receives between two to four input prompts. These prompts set the device operating conditions, and include channel selection, analog input range if supported, oversampling ratio if supported, and sampling rate. Once the user has input these parameters, the program sends an initialization command to the USBTMC interface containing the specified operating parameters. A CSV file is then created, and the DAQ resolution, analog range, and sample rate written to the header of the file. Additionally, the sample and channel number column headers are written to the file.

Stage 3: Data retrieval. This stage of the program handles the retrieval of data from the DAQ and saves this data to a file. Before beginning data collection, the user is prompted to begin data collection, allowing data collection to be manually initiated at the desired time. Once the prompt is received, the host 
sends a command to the DAQ to begin data collection. The program then continually sends data request commands, receives data, and writes the data to file. A second thread is used to simultaneously check for user input to end data collection; once this input is received, the thread alerts the main thread and exits. The main thread then sends a data collection end request and continues to receive data from the device until it receives an end of message flag.

The console application handles the resetting and buffer clearing of the STM32 USB DAQ. Therefore, the console application can be used to collect data regardless of what state the DAQ is in when the application first begins. The console application also handles error checking for user inputs; however, it cannot determine whether a selected sampling rate aligns with the DAQ ADC sampling timer, or whether this sampling rate is within the range of capabilities of the DAQ without losing data. For this reason, it is necessary to test a specific setup before assuming that collected data is valid. Instructions on how to use the console application can be seen in Appendix H.

\subsubsection{MATLAB Data Processing}

MATLAB is used to process and plot the rotordynamic data collected from the STM32 USB Data Acquisition System. This data processing and plotting is implemented as a group of MATLAB functions intended to be used with the MATLAB command window. Processing of the data occurs in three steps:

1. The data is read into MATLAB from the CSV file. The channel data is converted from raw binary code into a voltage level using the formula: 


$$
\text { Voltage }=\text { Binary Code } \times \frac{\text { Full Scale Range }}{2^{\text {Resolution }}}
$$

This equation is derived from the ADC transfer functions provided by AD760x data sheets. In addition to these voltages, the sample numbers are converted into timestamps by dividing by the sample rate.

2. A MATLAB object is created using the TestObject class definition, as with the National Instruments CompactDAQ [3]. The object is initialized using the sample rate retrieved from the CSV file, as well as user specified channels numbers, maximum rotor speed, and maximum sampling duration.

3. The voltage data is split into windows and passed to the plotData method of the object. These windows are processed and fill the remaining properties of the object with rotordynamic data. Once processing is complete, the newly processed rotordynamic amplitude, phase, and speed data is ready to be plotted and analyzed.

The processed data can be input into any of the MATLAB rotordynamic plotting functions for analysis. The plots that can be created using these functions include $2 \mathrm{D}$ and 3D orbit plots, Bode plots, a polar plot, and a cascade plot. Additionally, signal plots can be created for the raw voltage signals. The functions used for creating these plots are derived from the plotData method of the TestObject [3]. All MATLAB data processing and plotting functions can be seen in Appendix F. 


\section{Chapter 6: NI CompactDAQ and STM32 USB DAQ Attributes Comparison}

\subsection{Hardware Comparison}

The National Instruments CompactDAQ and the STM32 USB Data Acquisition System perform similar tasks that are achieved through different designs. A physical comparison between these two DAQs can be seen in Figure 13. Despite both DAQs having a relatively small footprint (when compared to other systems, such as the ADRE 408), they are dimensionally very different. The CompactDAQ nominally measures 3.47 inches long, 6.28 inches wide, and 4.24 inches tall $[6,21]$. In comparison, the STM32 USB DAQ, when using vertically facing BNC connectors, nominally measures 3.46 inches long, 5.28 inches wide, and 1.46 inches tall. Additionally, the CompactDAQ electronics are encased, while the STM32 USB DAQ electronics are exposed.

Although both DAQs utilize similar hardware components, they still differ significantly. Both DAQs utilize a USB 2.0 connection to send data to a computer; however, the

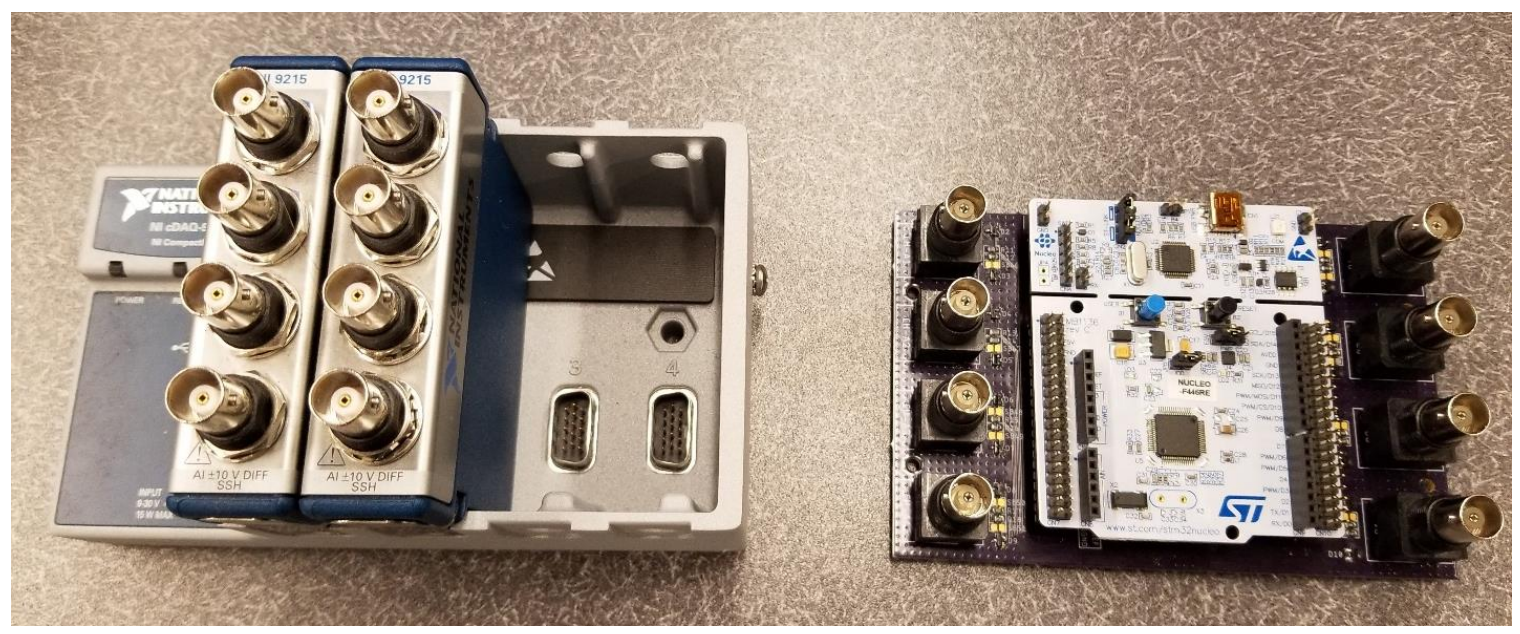

Figure 13. Physical comparison between the NI CompactDAQ (left) and the STM32 USB DAQ (right). 
CompactDAQ is capable of a USB high-speed connection, allowing for a data transfer rate of $480 \mathrm{Mb} / \mathrm{s}$, while the STM32 USB DAQ is only capable of a USB full-speed connection, allowing for a data transfer rate of $12 \mathrm{Mb} / \mathrm{s}$. Additionally, both DAQs can read analog data from up to 8 channels simultaneously. Each of these channels has a differential input that can take measurements up to \pm 10 volts. However, the CompactDAQ uses two 16-bit ADCs, each with 4 channels, while the STM32 USB DAQ uses one 18-bit ADC. Finally, the CompactDAQ requires an external power source to operate; conversely, the STM32 USB DAQ can be configured to be powered through either a USB connection (either the STLink USB port or the expansion board USB port), or through some external power source. A summary of these comparisons, as well as the physical comparisons discussed previously, can be seen in Table 4 .

Table 4. Hardware comparison between the NI CompactDAQ and the STM32 USB DAQ. Although the analog to digital converter used by the STM32 USB DAQ is capable of a sampling rate of $200 \mathrm{kSPS}$ per channel, this sampling rate cannot be achieved due to board design limitations.

\begin{tabular}{|l|c|c|}
\hline \multicolumn{1}{|c|}{ Attributes } & NI CompactDAQ & STM32 USB DAQ \\
\hline Length (Nominal) & $3.47 \mathrm{in}$ & $3.46 \mathrm{in}$ \\
\hline Width (Nominal) & $6.28 \mathrm{in}$ & $5.28 \mathrm{in}$ \\
\hline Height (Nominal) & $4.24 \mathrm{in}$ & $1.46 \mathrm{in}$ \\
\hline Enclosure & Yes & $\mathrm{No}$ \\
\hline USB Data Transfer Rate & $480 \mathrm{Mb} / \mathrm{s}$ & $12 \mathrm{Mb} / \mathrm{s}$ \\
\hline ADC Resolution & $16 \mathrm{bits}$ & $18 \mathrm{bits}$ \\
\hline Analog Channels & 8 & 8 \\
\hline Analog Input Range & $\pm 10 \mathrm{~V}$ & $\pm 10 \mathrm{~V}$ \\
\hline Max Sampling Speed (Per Channel) & $100 \mathrm{kSPS}$ & $200 \mathrm{kSPS}$ \\
\hline Analog Input Type & Differential & Differential \\
\hline External Power Required & Yes & No \\
\hline
\end{tabular}




\subsection{Software Comparison}

The National Instruments CompactDAQ and STM32 USB Data Acquisition System utilize very different software. The CompactDAQ itself utilizes proprietary embedded software and cannot be modified. Similarly, the NI-DAQmx computer side driver is also propriety, and cannot be modified either. However, the NI-DAQmx driver provides an API that can be used across many different programming languages and applications [7].

In contrast to the CompactDAQ, the software used to control and read data from the STM32 USB DAQ had to be written. As discussed in section 5.3, the STM32 USB DAQ was programmed using FreeRTOS based code running two tasks: a USB task and an ADC task. These tasks controlled their respective peripherals using the library code discussed in section 5.2. Additionally, as discussed in section 5.5.1, a console application was written to communicate with the STM32 USB DAQ. This application utilizes the two USB host libraries discussed in section 5.4.

Despite these differences, both the CompactDAQ and the STM32 USB DAQ utilize the same MATLAB code to process collected data, with the only difference being the means by which MATLAB obtains the data. The Rotor Kit App is used to collect and process data from the CompactDAQ in real time. In contrast, the MATLAB data processing function discussed in section 5.5.2 reads and processes STM32 USB DAQ data from a CSV file created by the console application. Both methods utilize a MATLAB TestObject: the plotData method is used to process the data. This processed data is stored in variables and arrays contained within the object. 


\section{Chapter 7: Experiments using the STM32 USB Data Acquisition System}

\subsection{STM32 USB Data Acquisition System Initial Measurements Verification}

Before the STM32 USB Data Acquisition System could be used to measure rotordynamic data, it needed to be tested in a controlled manner to ensure that collected data was valid. This was achieved using one of the signal generators in the Cal Poly Vibration Lab. These signal generators can produce sine waves, square waves, triangle waves, sawtooth waves, and random noise. To test the STM32 USB DAQ, the signal generator was set to produce a $100 \mathrm{~Hz}$ wave with a peak-to-peak magnitude of 2 volts and no offset. Channel 1 of the DAQ was connected to the output of the signal generator, and data was collected for sine, square, triangle, and sawtooth wave outputs. A 20millisecond period for each of these waves is shown in Figure 14. As can be seen, the period of all four signals is as expected, indicating that the DAQ is capable of reconstructing the shape of a signal. To determine any scaling and offset of the signal measured, the peak voltages of the sine wave were averaged. Using MATLAB, the average positive and negative peak voltages were found to be 1.0017 and -1.0006 volts, respectively. This translates into a worst-case average $0.12 \%$ scaling and $0.5 \mathrm{mV}$ offset of measured data, assuming the signal output by the signal generator is exact.

\subsection{Slow Roll Experiment}

Once the basic operation of the STM32 USB Data Acquisition System was verified, it was tested against the National Instruments CompactDAQ. To confirm the ability of the STM32 USB DAQ to collect valid data from the RK 4 Rotor Kit, a slow roll test was first performed. A BNC splitter was used to attach the Keyphasor ${ }^{\mathrm{TM}}$, X, and Y proximity 

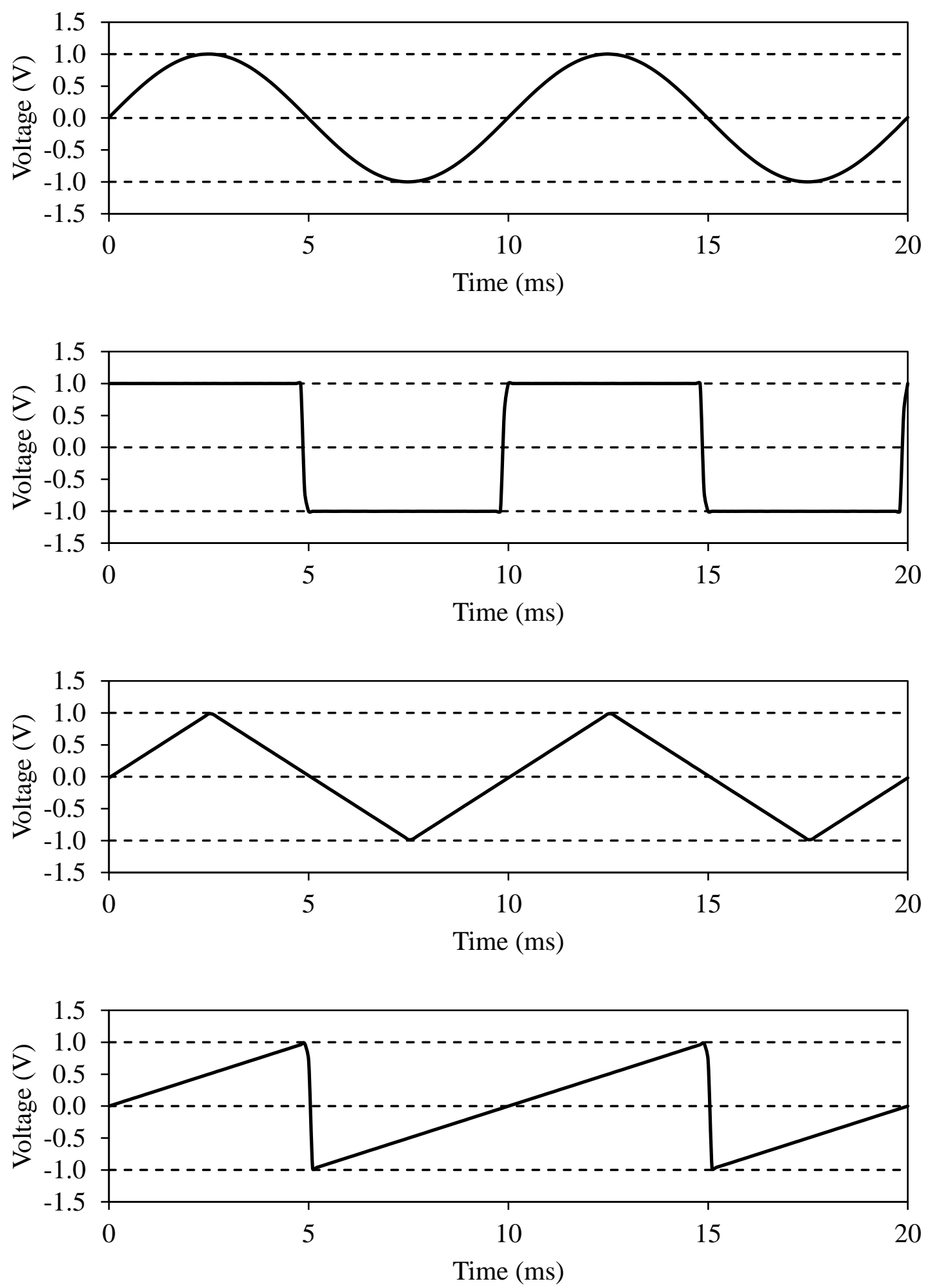

Figure 14. Test measurement of the signal generator output using the STM 32 USB DAQ. The signals measured, from top to bottom, are a sine wave, a square wave, a triangle wave, and a sawtooth wave. The dashed lines at $-1,0$, and 1 volts provide a frame of reference. 
sensor outputs on the top TK21 Oscilliscope Interface to first three analog input channels of both the CompactDAQ and the STM32 USB DAQ. The attachment of the proximity sensor outputs to both DAQs can be seen in Figure 15. Both data acquisition systems were connected to a charging laptop to control data collection.

However, when data collection was started, a significant problem was encountered with the STM32 USB DAQ. Pulses were being recorded at regular intervals in-between otherwise normal data. These pulses occurred across all three channels. However, these pulses were not present in the data recorded by the CompactDAQ. A 0.4 second interval comparison of data recorded from channel 1 of the CompactDAQ and channel 2 of the STM32 USB DAQ (both of which were connected to the X proximity sensor) is shown in Figure 16. As can be seen in the figure, the data recorded between pulses is almost

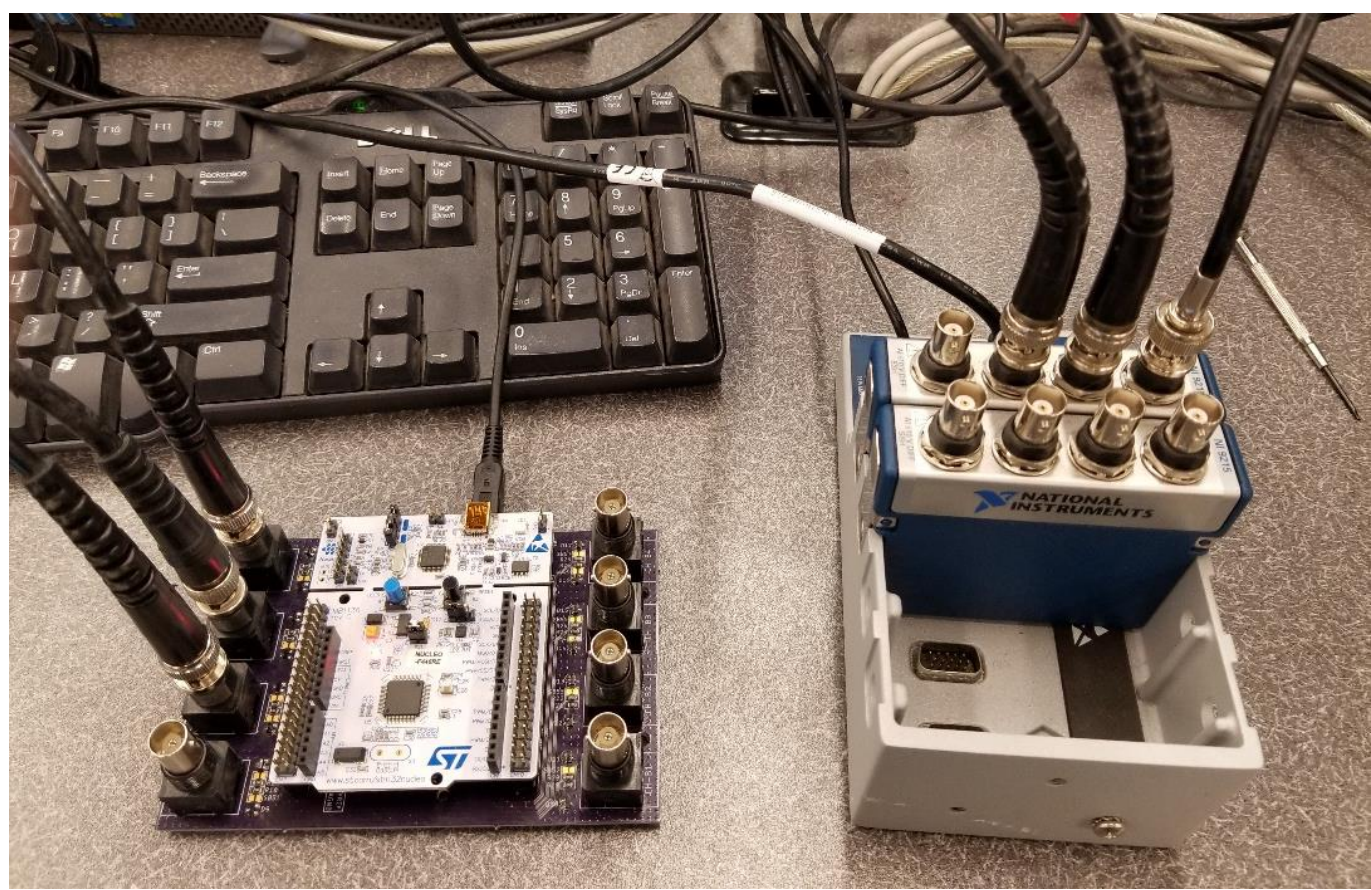

Figure 15. Rotor Kit proximity sensor connections to both the CompactDAQ and the STM32 USB DAQ. 


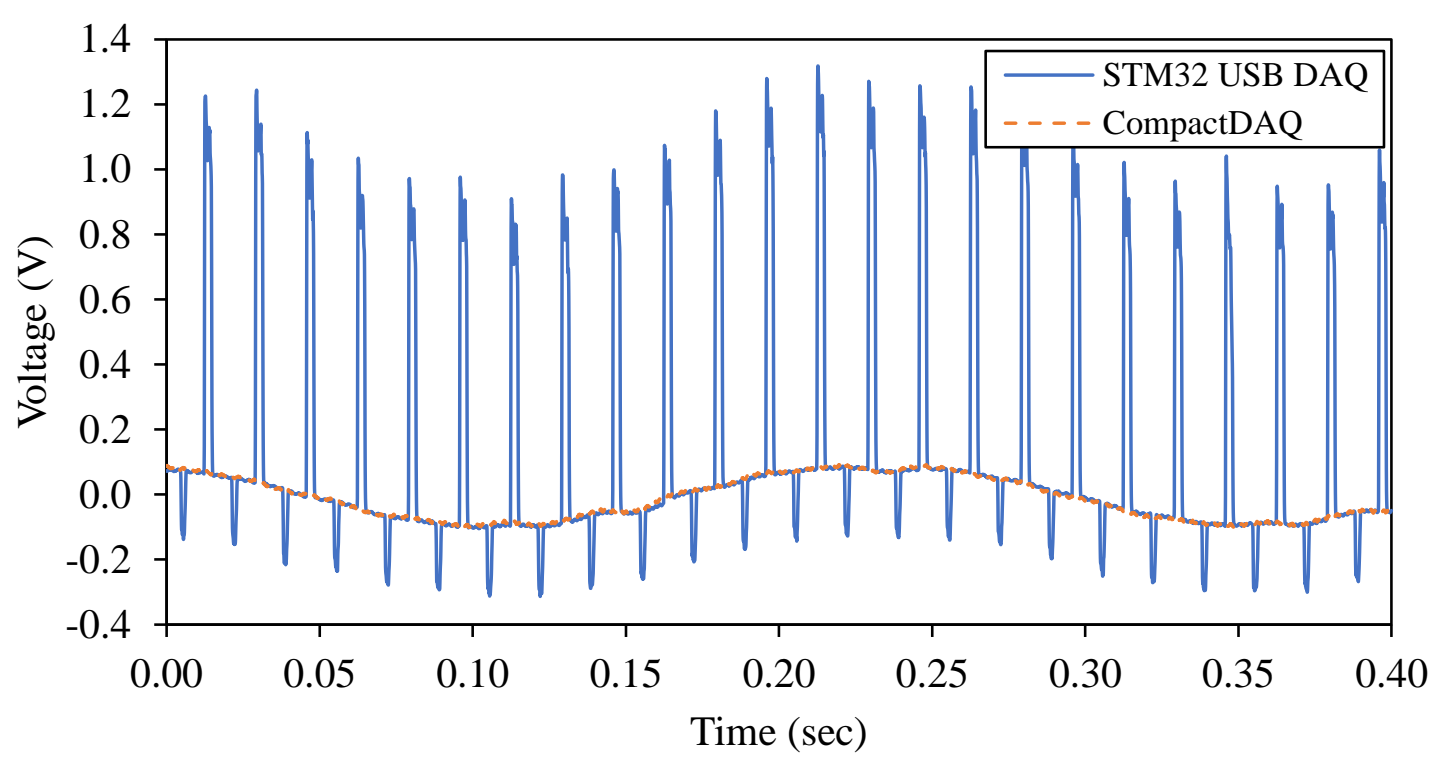

Figure 16. Raw voltage data collected from the RK 4 Rotor Kit using the STM32 USB DAQ and the CompactDAQ. Pulses occur at regular intervals in the STM32 USB DAQ data, but not the CompactDAQ data.

indistinguishable. Since the data recorded using the CompactDAQ should be valid (as determined from previous experiments) the data collected by the STM32 USB DAQ between pulses should be valid as well [3].

In contrast to this recorded data, the pulses disappeared when data were collected while the laptop was running on battery power and the CompactDAQ was disconnected. To best compare this pulse free data, slow roll data was collected using the STM32 USB DAQ and the CompactDAQ separately. A 0.4 second interval of data recorded from channel 1 of the CompactDAQ and channel 2 of the STM32 USB DAQ is shown in Figure 17. As can be seen in the figure, both data sets are almost identical, in contrast to the data shown in Figure 16.

This discrepancy between these two sets of data collected seem to indicate that the issue arises from an isolation problem. A common isolation problem with analog to digital 


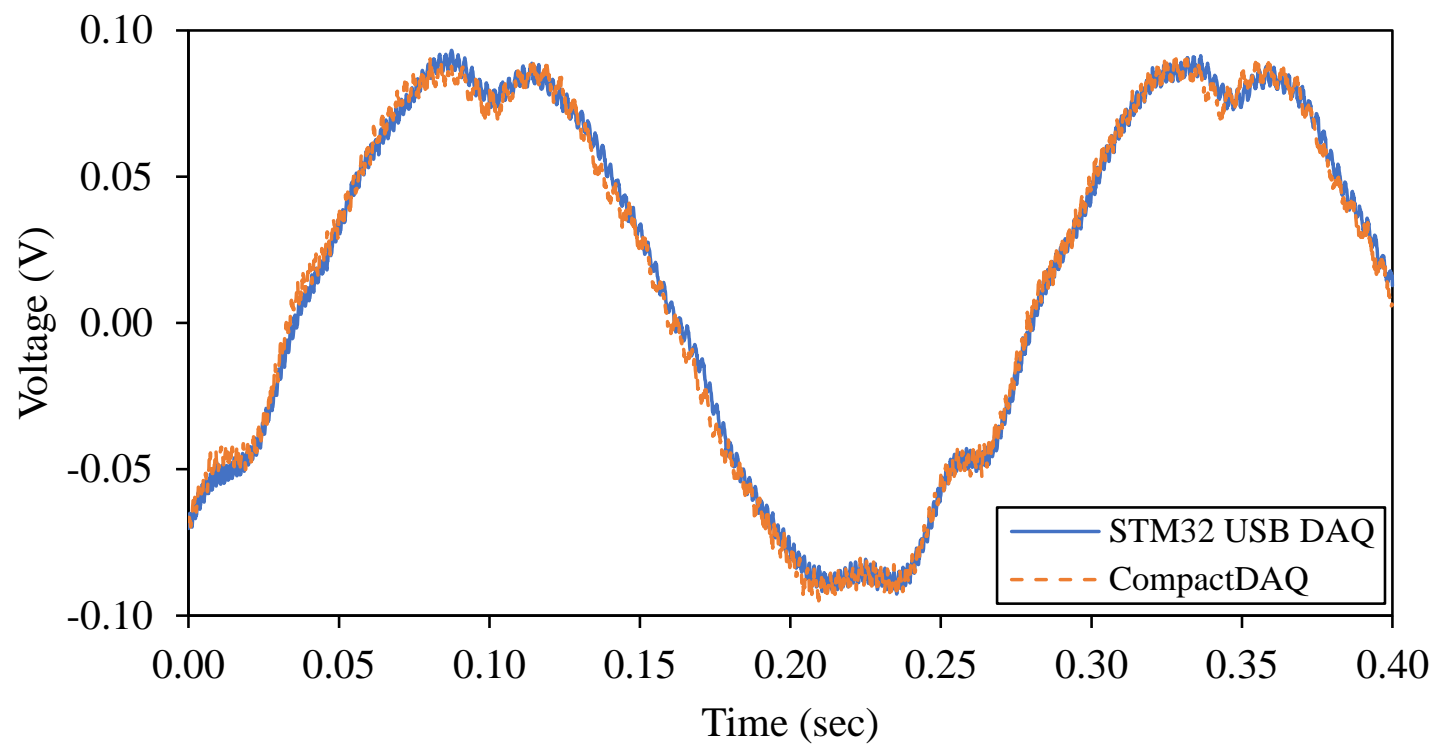

Figure 17. Raw voltage data collected from the RK 4 Rotor Kit using the STM 32 USB DAQ and the CompactDAQ. The pulses previously seen in the STM32 USB DAQ data disappear when the system is isolated from all sources except the sensor inputs.

converters results from the presence of a ground loop. Ground loops are created when a single ground on a device has two or more connections to the same external ground. These connections create a conductive loop that acts as an antenna and is therefore highly sensitive to interference. Any interference picked up by the loop creates currents, causing voltage fluctuations due to a non-zero resistance in the loop conductors. The result is an unstable reference ground, causing measurements of analog signals to be unstable as well [22].

Although the analog inputs to the STM32 USB DAQ are not directly connected to ground, a similar problem may exist if neither the ADC nor the sensors are isolated. Since the datasheet for the AD7609 ADC does not state that it is isolated, the assumption can be made that it is not (input isolation is a feature on some ADCs but not others and is therefore usually listed if incorporated). Similarly, neither the RK 4 Rotor Kit datasheet 
nor the TK21 Oscilliscope Interface datasheet state that the sensor outputs are isolated. If the ADC and sensors are not isolated, then an indirect ground connection between the two would exist. Therefore, when either connecting to a charging laptop, or connecting to a laptop with self-powered devices attached (such as the CompactDAQ), a loop would be formed, creating an unstable reference ground. This hypothesis is supported by the pulses seen in Figure 16: the magnitude of the pulses seem to follow the same trend as the signal they disrupt, indicating that the reference ground potential pulses in a regular manner.

In contrast to the STM32 USB DAQ, the analog to digital converters in the NI 9215 modules used with the CompactDAQ incorporate double isolation [8]. This isolation accounts for the pulse-free data collected by the CompactDAQ, even when sharing proximity sensor connections with the STM32 USB DAQ.

This ground loop problem can be fixed by cutting the loop. The loop can be cut by isolating either the digital side or the analog side of the ADC. Without redesigning the Data Acquisition Expansion Board, isolation can be achieved by isolating either the USB connection (and power supply if separate) or the analog inputs. It is simpler to isolate USB connection than it is to isolate all eight analog inputs; therefore, the decision to use a USB isolator with the STM32 USB DAQ was made.

Three USB isolators were selected for testing: the HiLetgo USB Isolator, the SMAKN USB Isolator, and the EZSync USB Isolator (see Figure 18). All three isolators are 


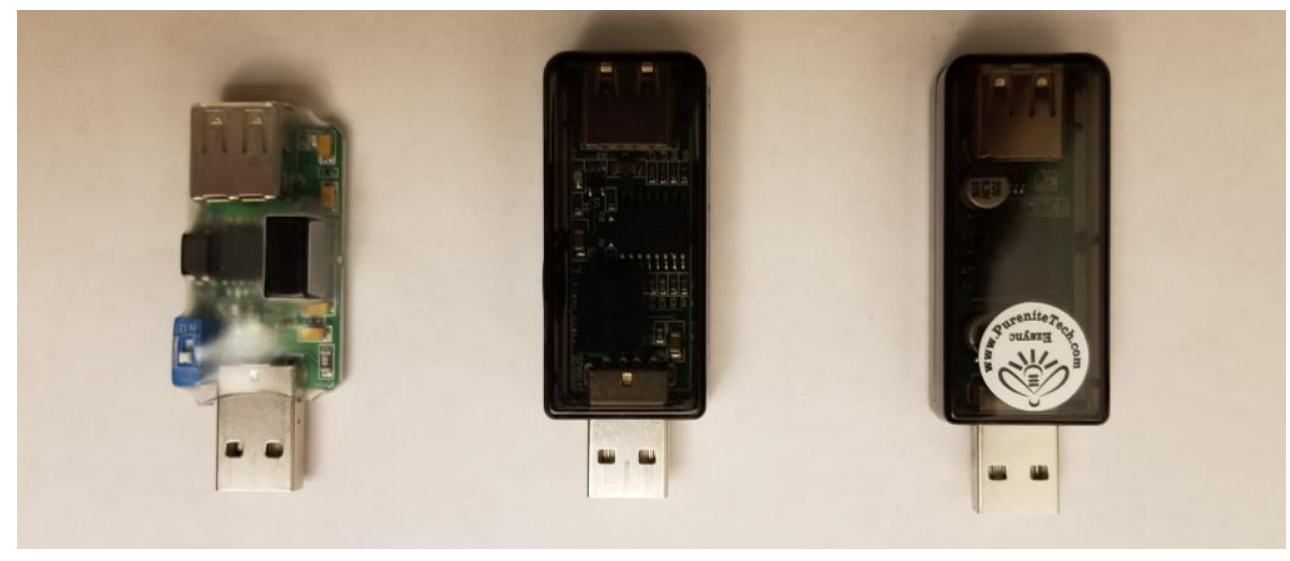

Figure 18. Image of the HitLetgo (left), SMAKN (middle), and EZSync (right) USB isolators used with the STM32 USB DAQ.

capable of USB full-speed communication and can power a connected device. The isolation capabilities of each of these three isolators were tested by repeating the slow roll experiment while the laptop was charging. Data was also collected using the CompactDAQ to provide a frame of reference. A 0.4 second window of data collected from the X proximity sensor using each USB isolator is shown in Figure 19. As can be seen, all three isolators break the loop previously formed, and allow for data collection comparable to that of the CompactDAQ. All three isolators produce almost identical results: over the range of data shown, the maximum difference between the STM32 USB DAQ data and the CompactDAQ data was $0.012 \mathrm{~V}$ for the HiLetgo, 0.014 for the SMAKN, and $0.010 \mathrm{~V}$ for the EZSync. As such, the EZSync USB isolator was used for the single plane imbalance experiment.

\subsection{Single Plane Imbalance Experiment}

Once the STM32 USB Data Acquisition System was collecting useable data from the Rotor Kit, further comparison against the CompactDAQ was performed to confirm that it can viably be used for rotordynamic experimentation. This comparison was achieved by 

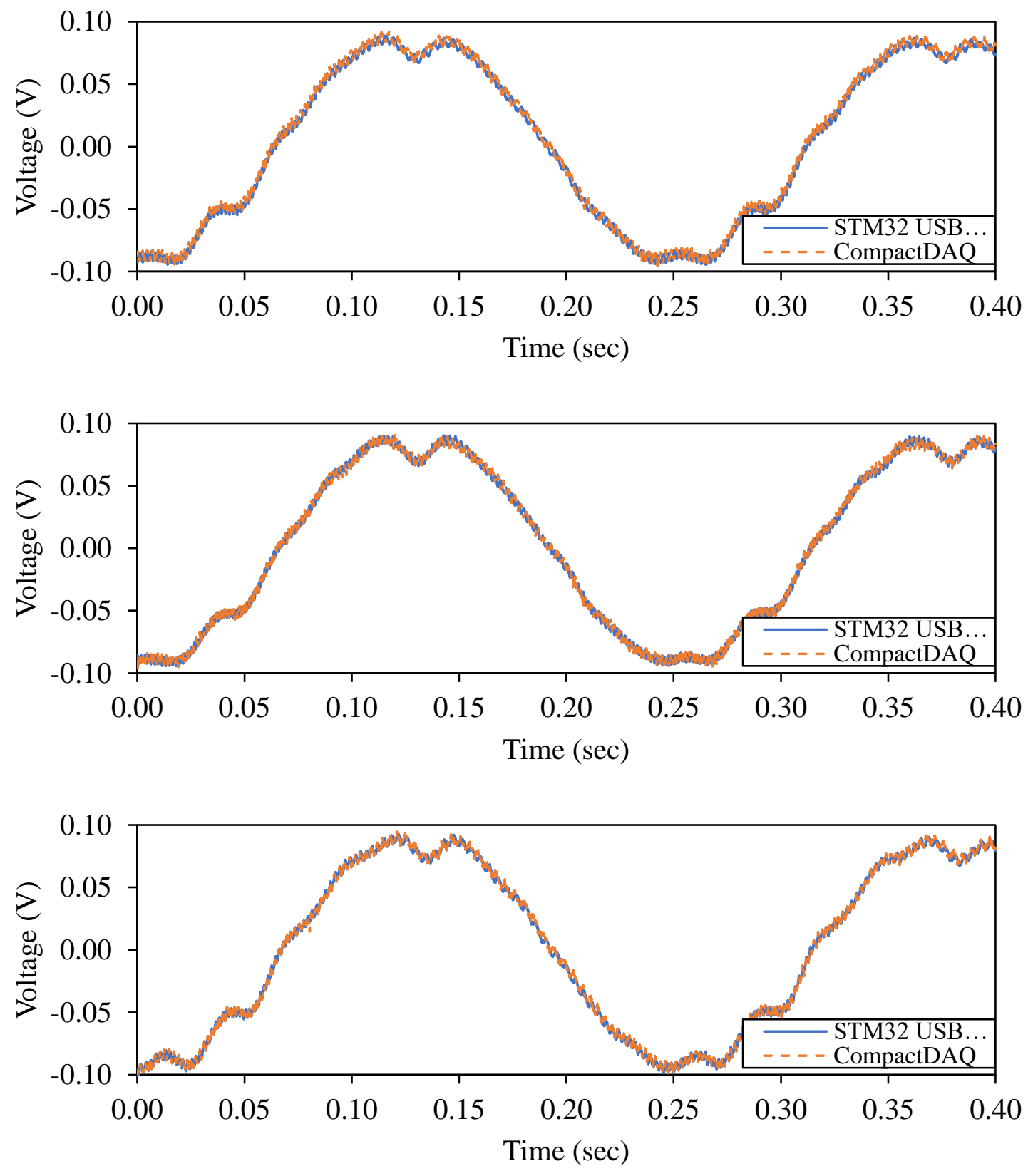

Figure 19. Slow roll test results using the HiLetgo (top), SMAKN (middle), and EZSync (bottom) USB isolators. Each isolator was compared against the CompactDAQ.

performing a single plane imbalance experiment using the RK 4 Rotor Kit. A rotor mass was attached near the center of the rotor shaft, and a weight added to cause an imbalance. One set of $\mathrm{X}$ and $\mathrm{Y}$ phasors was moved next to but not touching the rotor mass. Finally, 
the motor speed controller was adjusted to set the maximum motor speed to 3000 RPM and ramp rate to $4000 \mathrm{RPM} / \mathrm{min}$.

Next, using the console application, the STM32 USB DAQ was set to collect data from channels 1 through 3 at a rate of $10 \mathrm{kSPS}$, with an analog range of \pm 10 volts and oversampling ratio of 1 . Using the Rotor Kit App, the CompactDAQ was set also set to collect data a rate of $10 \mathrm{kSPS}$. The app was additionally set with a sampling duration of 120 seconds, a window size of 5000 samples, and a speed limit of 3000 RPM. Once the setup was complete, data collection was begun for both DAQs, and the Rotor Kit ramped up to its maximum set speed.

Once data collection was complete, the data collected by the STM32 USB DAQ was imported into MATLAB and processed. The Bode plots produced by horizontal data from both the CompactDAQ and the STM32 USB DAQ are shown in Figure 20. As can be seen, the amplitude and phase lag data show similar results for both DAQs. The two data sets shown in the amplitude plot are almost indistinguishable, showing very little difference between data collected by the STM32 USB DAQ and the CompactDAQ throughout the entire speed range, and it can be seen from both sets of data that the first natural frequency of the experimental setup is approximately 2040 RPM. The phase plot also shows very little difference between the data sets at speeds higher than approximately 1000 RPM; however, at slower speed, there are some discrepancies. This discrepancy is partially a result of a difference in usable data points, as MATLAB was unable to calculate the phase at slower speeds across both data sets. At the phases that were calculated, however, both data sets show similar trends; in particular, they show the 180-degree phase change that occurs at the first natural frequency. 

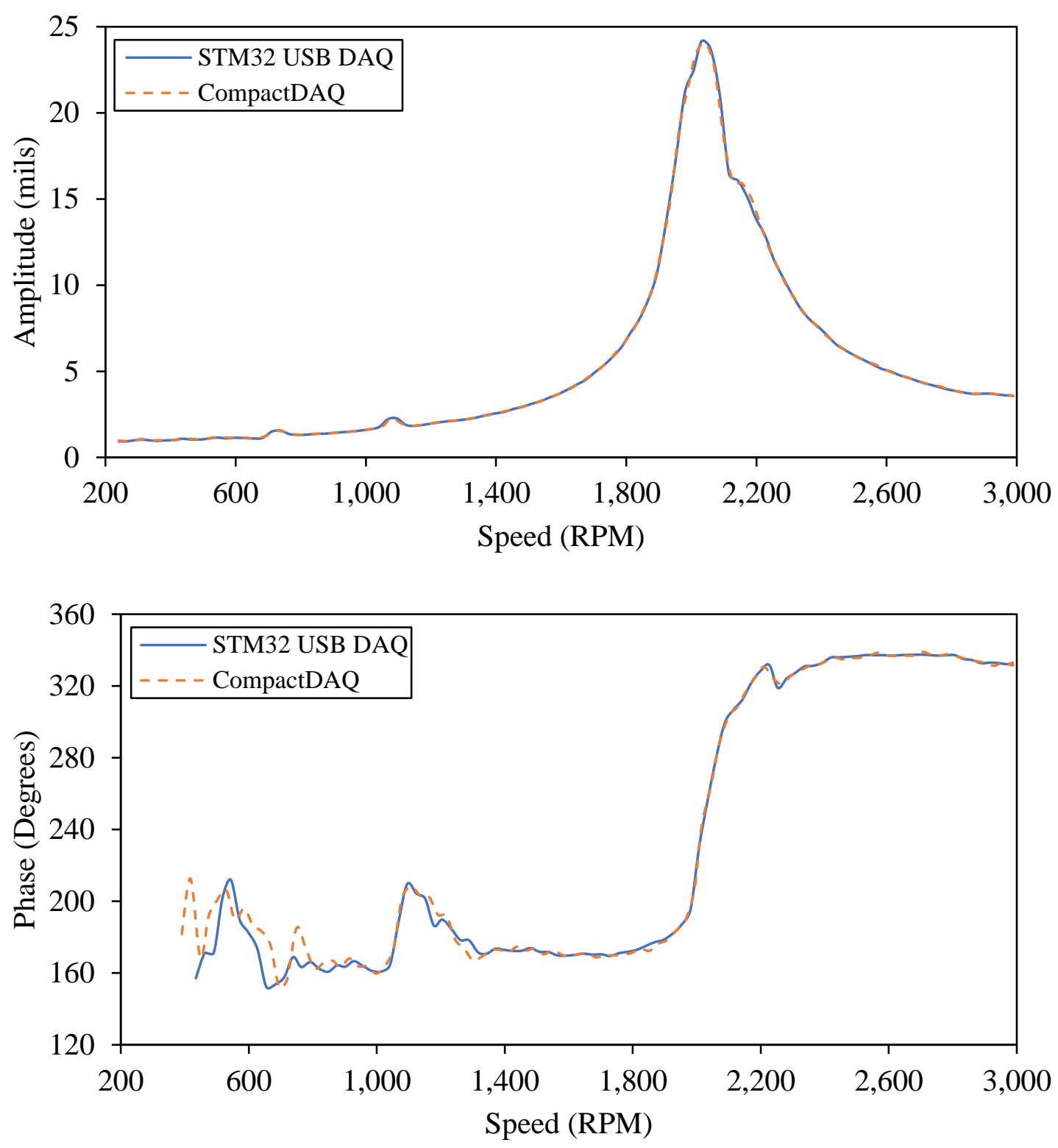

Figure 20. Bode plots showing the amplitude and phase lag in the horizontal direction. These results were analyzed using data collected from the CompactDAQ and the STM32 USB DAQ during the single plane imbalance experiment.

In addition to the Bode plots, a polar plot produced from the same horizontal amplitude and phase data can be seen in Figure 21. Unlike the Bode plots, the data collected by the STM32 USB DAQ can be distinguished from the data collected by the CompactDAQ at 


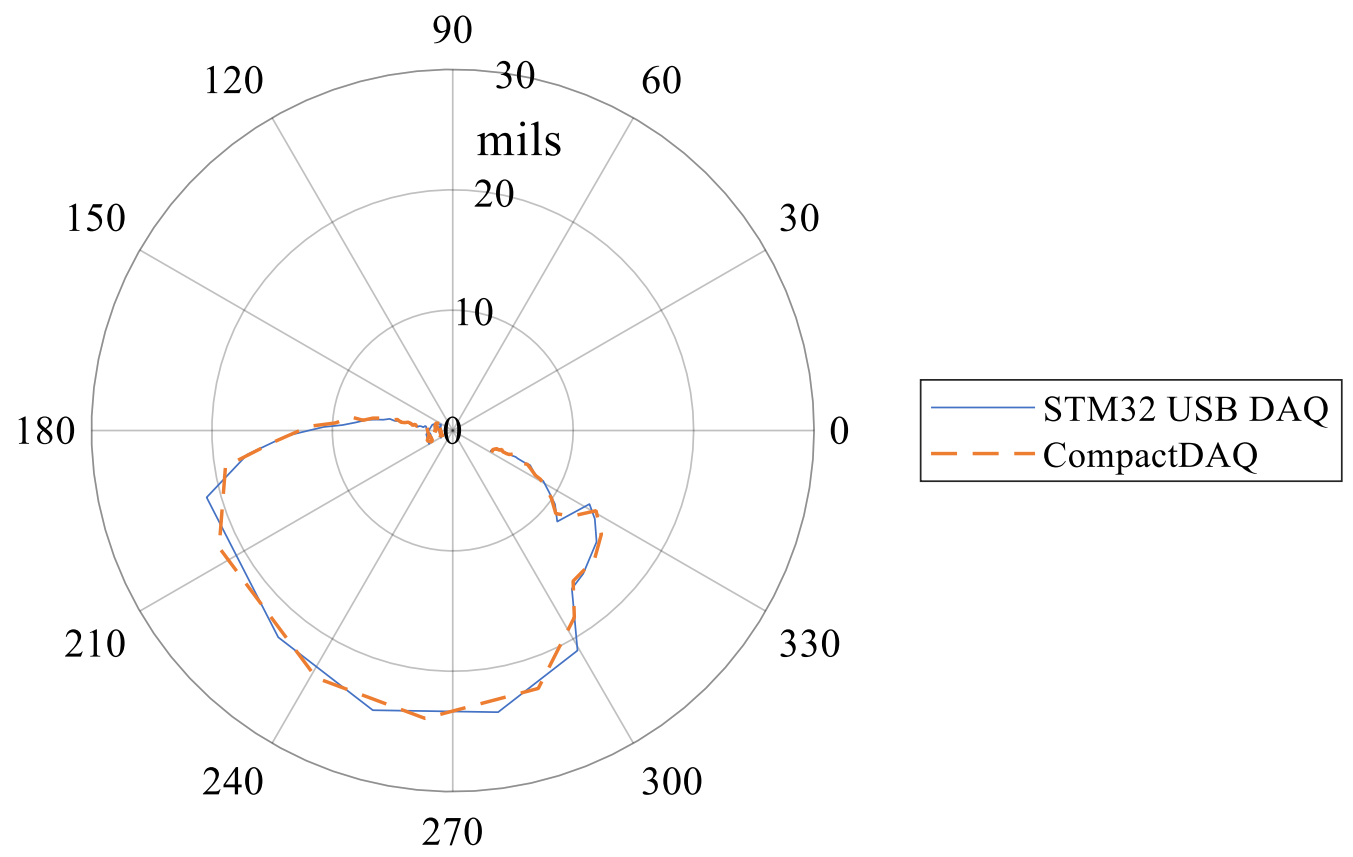

Figure 21. Uncompensated polar plot showing amplitude with respect to phase lag in the horizontal direction.

the higher amplitudes. This is due to both the lower resolution of the graph at these amplitudes, as well as the difference in phase data points between the two data sets. Despite this, there is very little difference between the two data sets, in agreement with the Bode plots.

Finally, full spectrum cascade plots produced from both sets of data can be seen in Figure 22. Both plots show a very large $-1 \mathrm{X}$ component of vibration, along with a smaller $1 \mathrm{X}$ component and very minor $\pm 2 \mathrm{X}$ components. As with the polar plot, the natural frequency of approximately 2040 RPM can be seen in agreement with the Bode plots. 

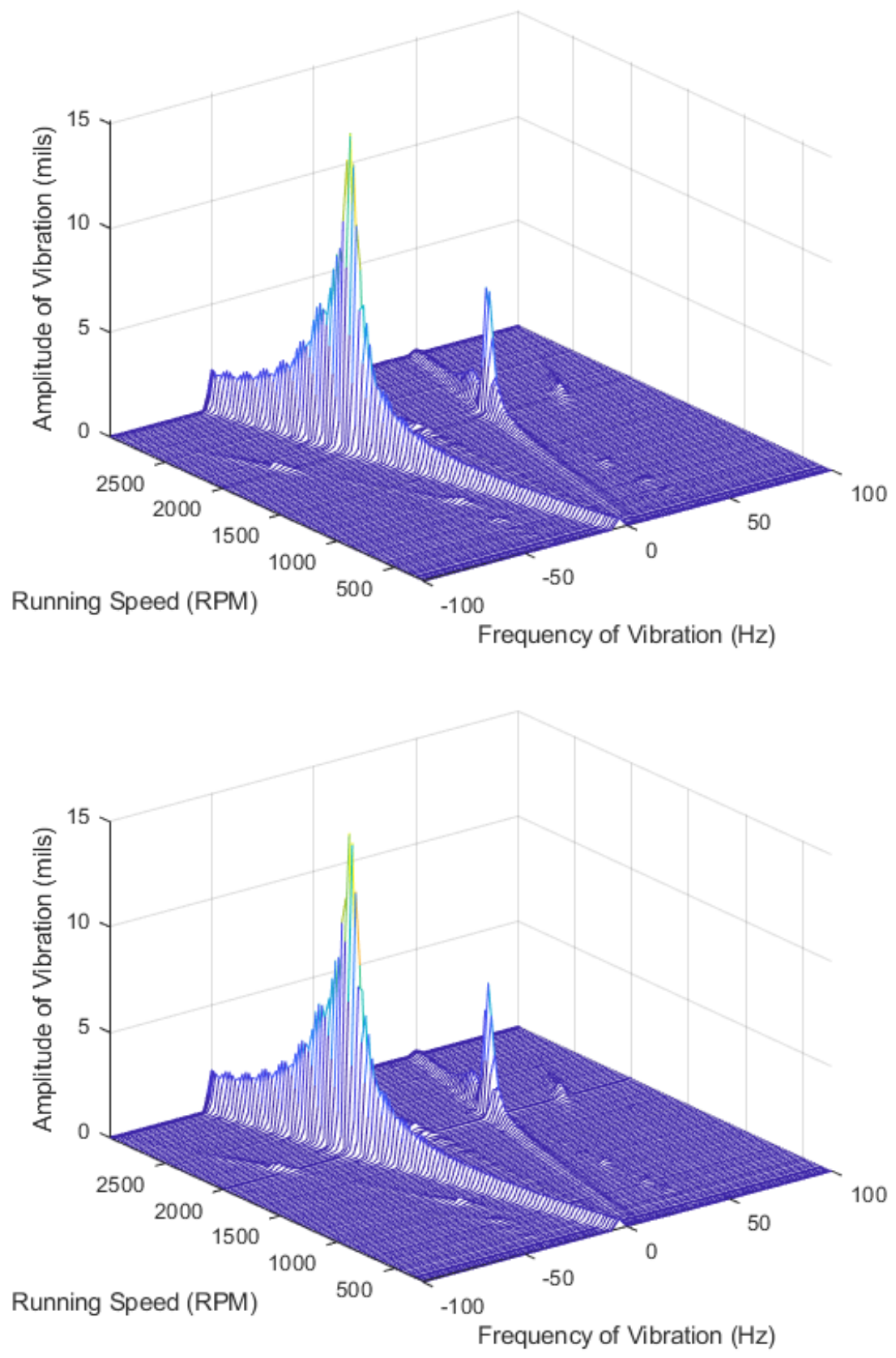

Figure 22. Full spectrum cascade plots produced from data collected by the STM 32 USB DAQ (top) and the CompactDAQ (bottom). 


\section{Chapter 8: Discussion and Conclusion}

\subsection{Data Acquisition System Design Summary}

The STM32 USB Data Acquisition System is a low cost DAQ capable of accurately measuring analog signals. This DAQ was designed using an STM32 Nucleo-F446RE development board and incorporates an AD7609 differential analog to digital converter to collect data, and a USB 2.0 full-speed connection to send collected data to a host computer. The STM32 Nucleo code utilizes FreeRTOS, allowing for data to be simultaneously collected and transferred to the connected computer. A computer side console application was developed, allowing for the DAQ to be controlled. Both the microcontroller and computer side USB code adhere to the USB Test and Measurement Class specification, allowing the for the DAQ to be controlled as a test and measurement device. Collected data is saved to a CSV file and read into MATLAB for analysis.

\section{$\underline{8.2 \text { Experiment Summary }}$}

Experiments were performed using the RK4 Rotor Kit in the Cal Poly Vibrations Lab to compare the STM32 USB DAQ to the National Instruments CompactDAQ. Initial slow roll testing showed a significant discrepancy between the data collected by both DAQs in the form of periodic pulses. These pulses were produced when collecting data on a charging laptop and disappeared then the laptop power cord was unplugged. These results indicated that a loop was created when the laptop was charging, creating an interference sensitive antenna and causing voltage fluctuations due to induced currents. Isolating the USB connection between the laptop and the STM32 USB DAQ breaks the loop and corrects the problem. 
After correcting the problem, additional tests were performing using a single plane imbalance experiment. Data was collected by both DAQs while the Rotor Kit was ramped up to a speed of 3000 RPM. After analyzing the data using MATLAB, Bode plots, a polar plot, and full spectrum cascade plots were created using data collected by both DAQs. These plots showed very little difference between the data collected by the STM32 USB DAQ and the data collected by the CompactDAQ.

\section{$\underline{8.3 \text { Conclusion and Future Recommendations }}$}

The STM32 USB Data Acquisition System is a capable alternative to other available DAQs. Its low cost and transparency make it suitable for educational environments, where students can learn about the means through which data is collected as well as the purposes for which collected data is used. Additionally, its configurability allows for different setups to be used and tested. When used with a Rotor Kit and MATLAB, the STM32 USB DAQ allows for rotordynamic data to be collected and analyzed.

When connecting to a non-isolated computer or sensors, the design of the STM32 USB DAQ requires isolation of either the analog inputs or of the connection to the host computer to avoid a loop being formed. If design changes are made to the DAQ, it is recommended that this isolation is incorporated into the design. Isolation can be achieved through various means, including using an isolated ADC, adding isolation circuity between the ADC and the rest of the DAQ, or adding isolation circuitry to either the analog or the USB and power inputs to the DAQ. 


\section{REFERENCES}

[1] Data Acquisition Handbook, 3rd ed., Norton, MA: Measurement Computing Corporation, 2012.

[2] D. E. Bently and C. T. Hatch, Fundamentals of Rotating Machinery Diagnostics, B. Grissom, Ed., Minden, NV: Bently Pressurized Bearing Company, 2002.

[3] D. L. Baker, "Development of a Rotordynamic Signal Processing MATLAB Interface and a Two-Disk Rotor Model," 2017.

[4] RK 4 Rotor Kit, Minden, NV: Bently Nevada, 2016.

[5] TK21 Oscilloscope Interface, Minden, NV: Bently Nevada, 2007.

[6] NI cDAQ-9174: NI CompactDAQ Four-Slot USB Chassis, National Instruments, 2013.

[7] CompactDAQ Chassis, National Instruments, 2017.

[8] NI 9215 Datasheet, National Instruments, 2016.

[9] Universal Serial Bus Specification, 2000.

[10] UM1724 User Manual: STM32 Nucleo-64 Boards, STMicroelectronics, 2017.

[11] AN4879 Application Note: USB Hardware and PCB Guidelines Using STM32 MCUs, STMicroelectronics, 2018.

[12] USBLC6-2: Very Low Capacitance ESD Protection, STMicroelectronics, 2011. 
[13] AP2552/AP2553/AP2552A/AP2553A: Precision Adjustable Current-Limited Switches, Diodes Incorporated, 2014.

[14] UM1974 User Manual: STM32 Nucleo-144 Boards, STMicroelectronics, 2017.

[15] D. Brooks, "Splitting Planes for Speed and Power," Printed Circuit Design, December 2000.

[16] H. W. Ott, "Partitioning and Layout of a Mixed-Signal PCB," Printed Circuit Design, June 2001.

[17] D. Brooks, Crosstalk, Part 1: Understanding Forward vs Backward, Mentor Graphics, 2003.

[18] Data Sheet AD7609: 8-Channel Differential DAS with 18-Bit, Bipolar, Simultaneous Sampling ADC, Norwood, MA: Analog Devices, 2014.

[19] "Defined Class Codes," 15 June 2016. [Online]. Available: https://www.usb.org/defined-class-codes. [Accessed 2018].

[20] Universal Serial Bus Test and Measurement Class Specification (USBTMC), USB Implementors Forum, 2003.

[21] J. R. Ridgely, Using Tasks and Finite State Machines in C++, 2017.

[22] C Series Module with 4 BNC, National Instruments, 2007.

[23] Understanding Ground Loops, Gays Mills, WI: Building Automation Products, 2015. 


\section{Appendix A: Data Acquisition Expansion Board Schematic}
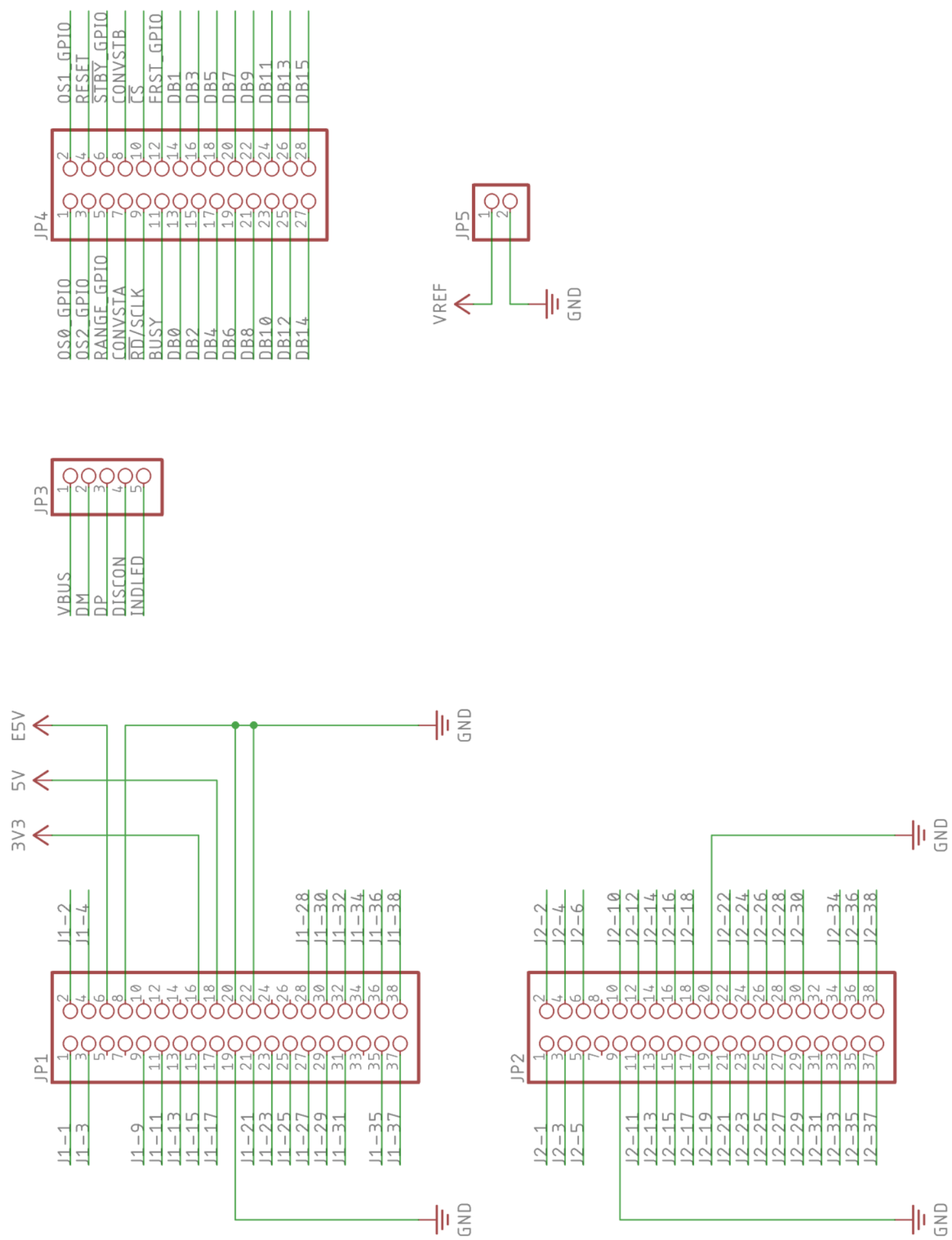

Figure A1. Schematic of the Data Acquisition Expansion Board headers. 


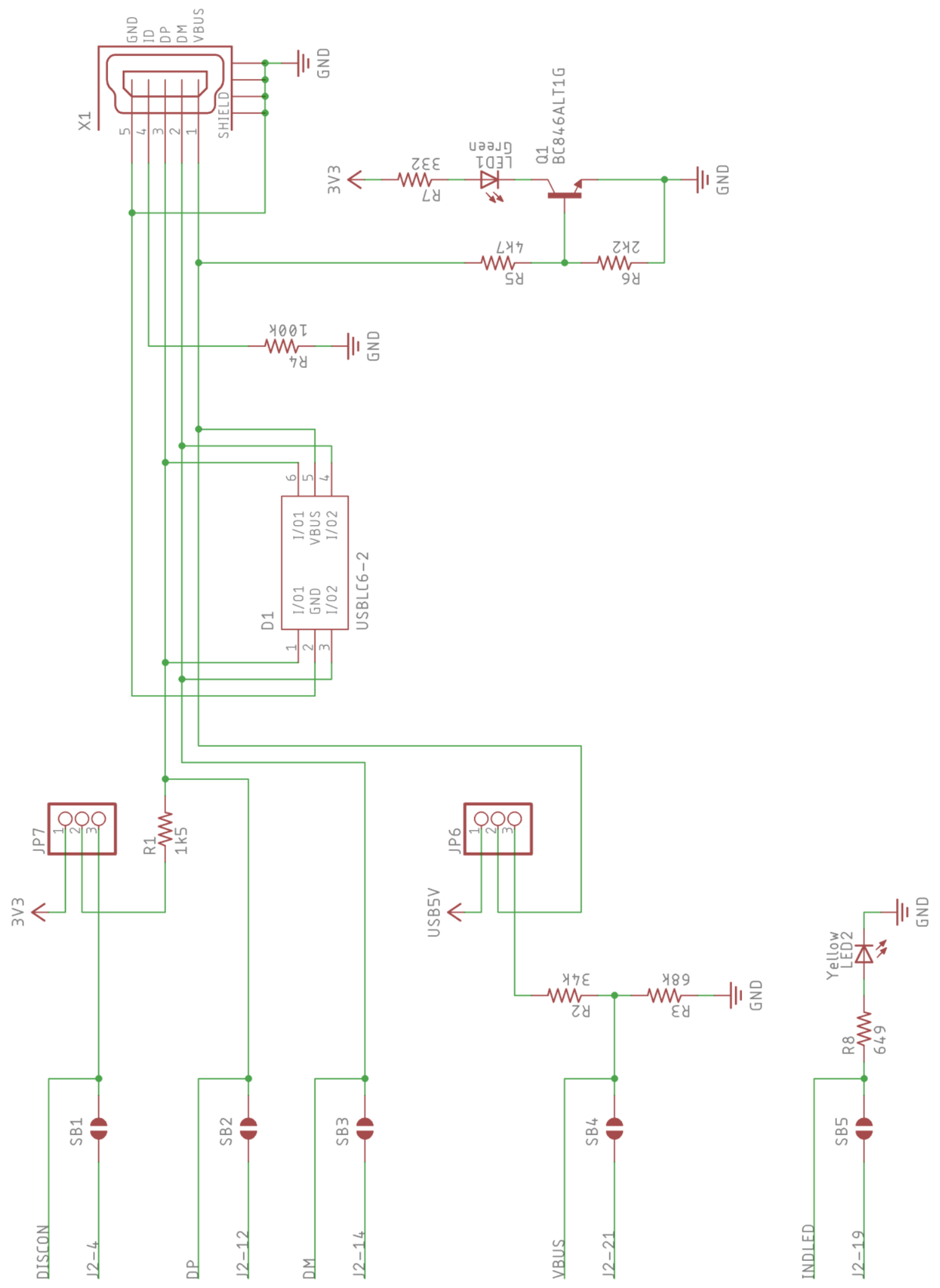

Figure A2. Schematic of the Data Acquisition Expansion Board USB port. 


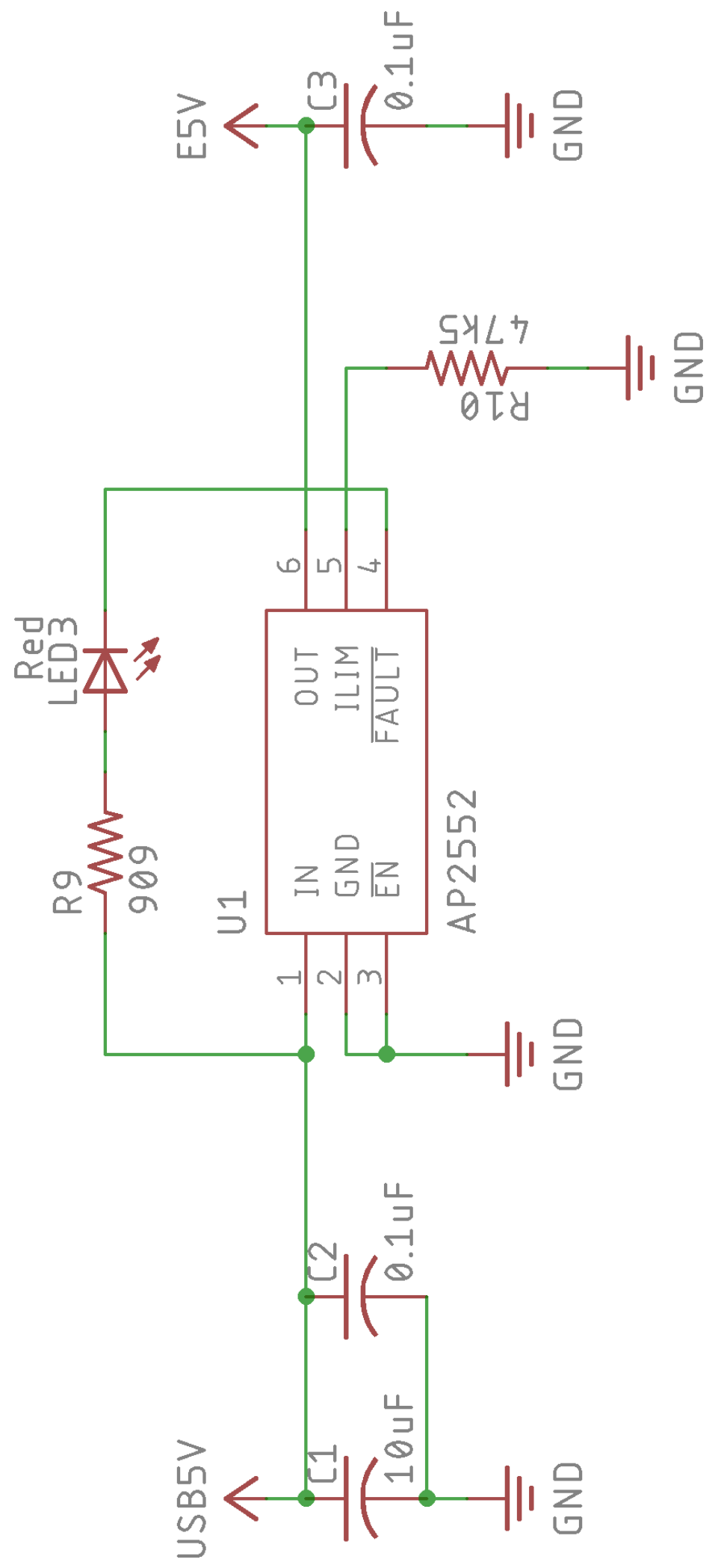

Figure A3. Schematic of the Data Acquisition Expansion Board current limiter. 


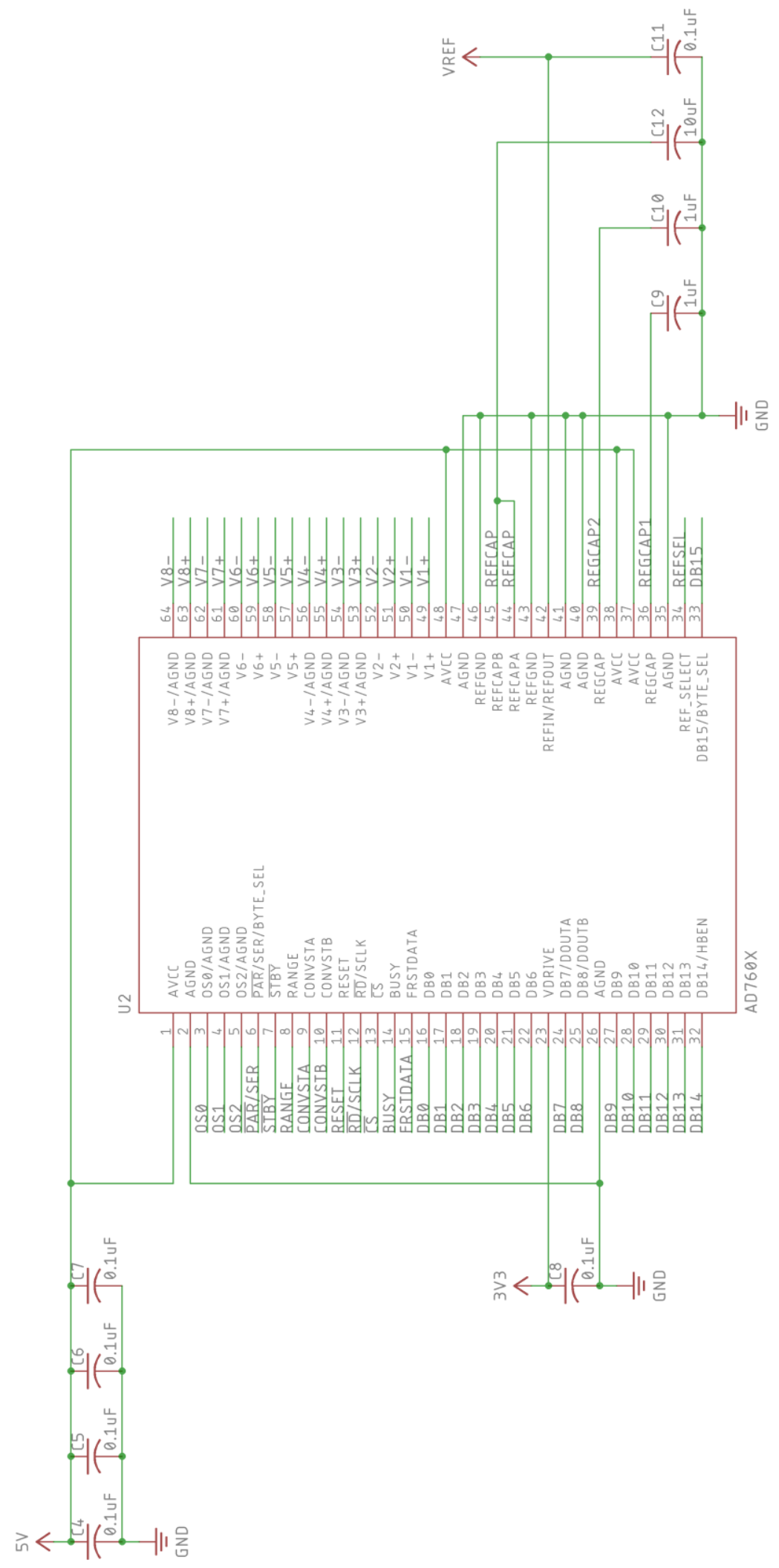

Figure A4. Schematic of the Data Acquisition Expansion Board ADC. 


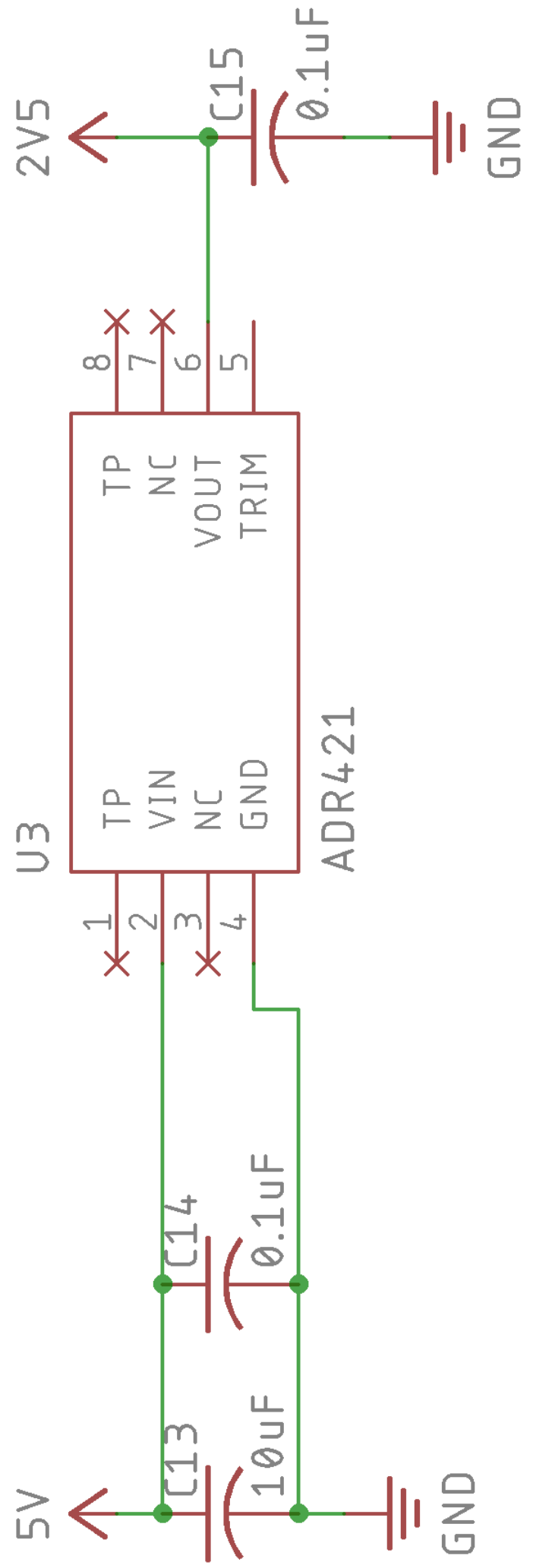

Figure A5. Schematic of the Data Acquisition Expansion Board voltage reference. 

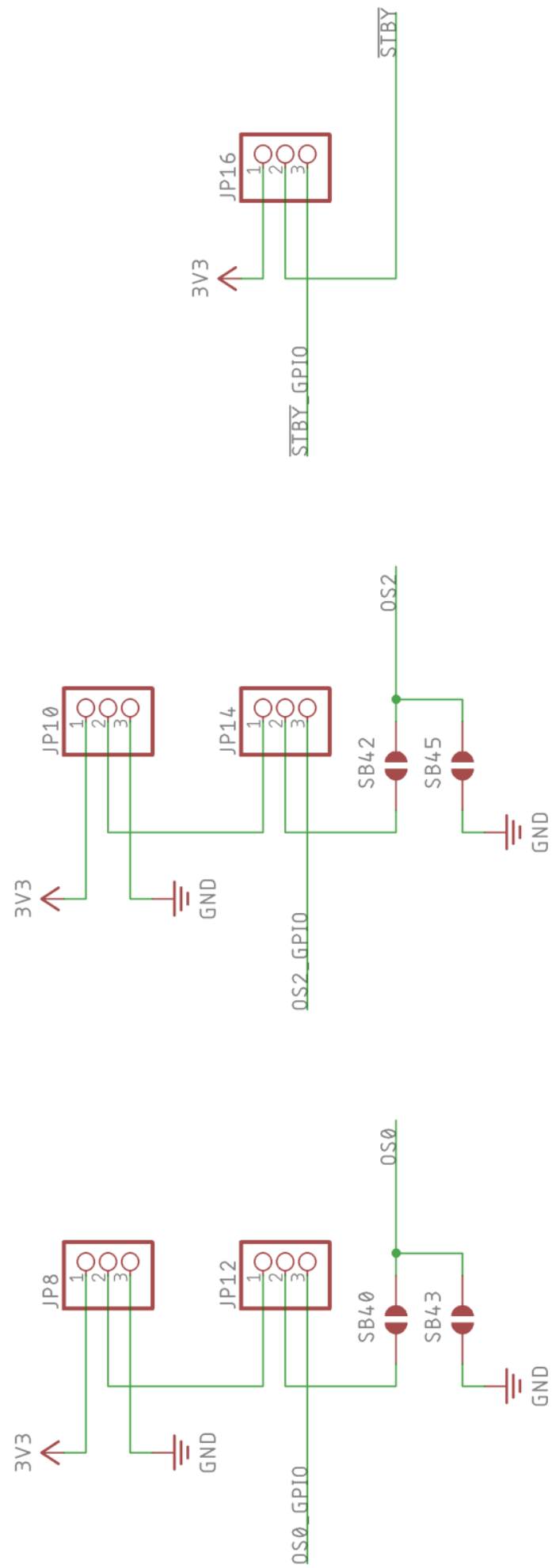
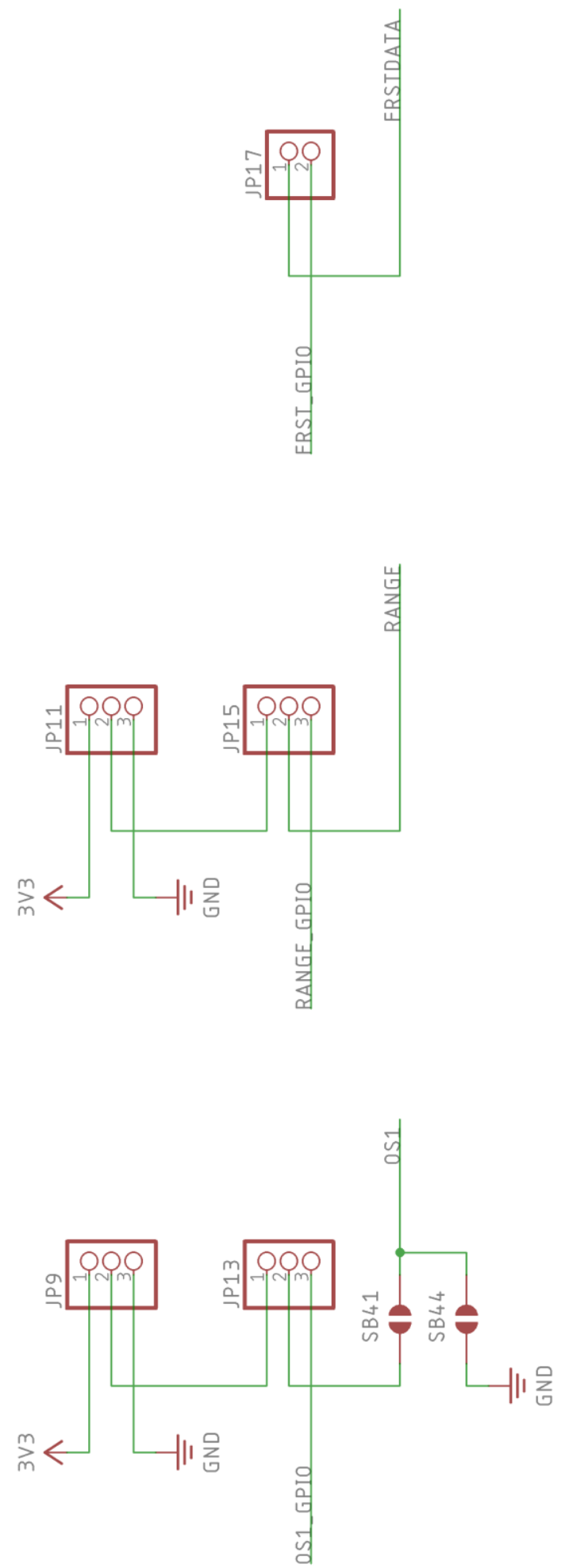

Figure A6. Schematic of the Data Acquisition Expansion Board ADC configuration jumpers. 

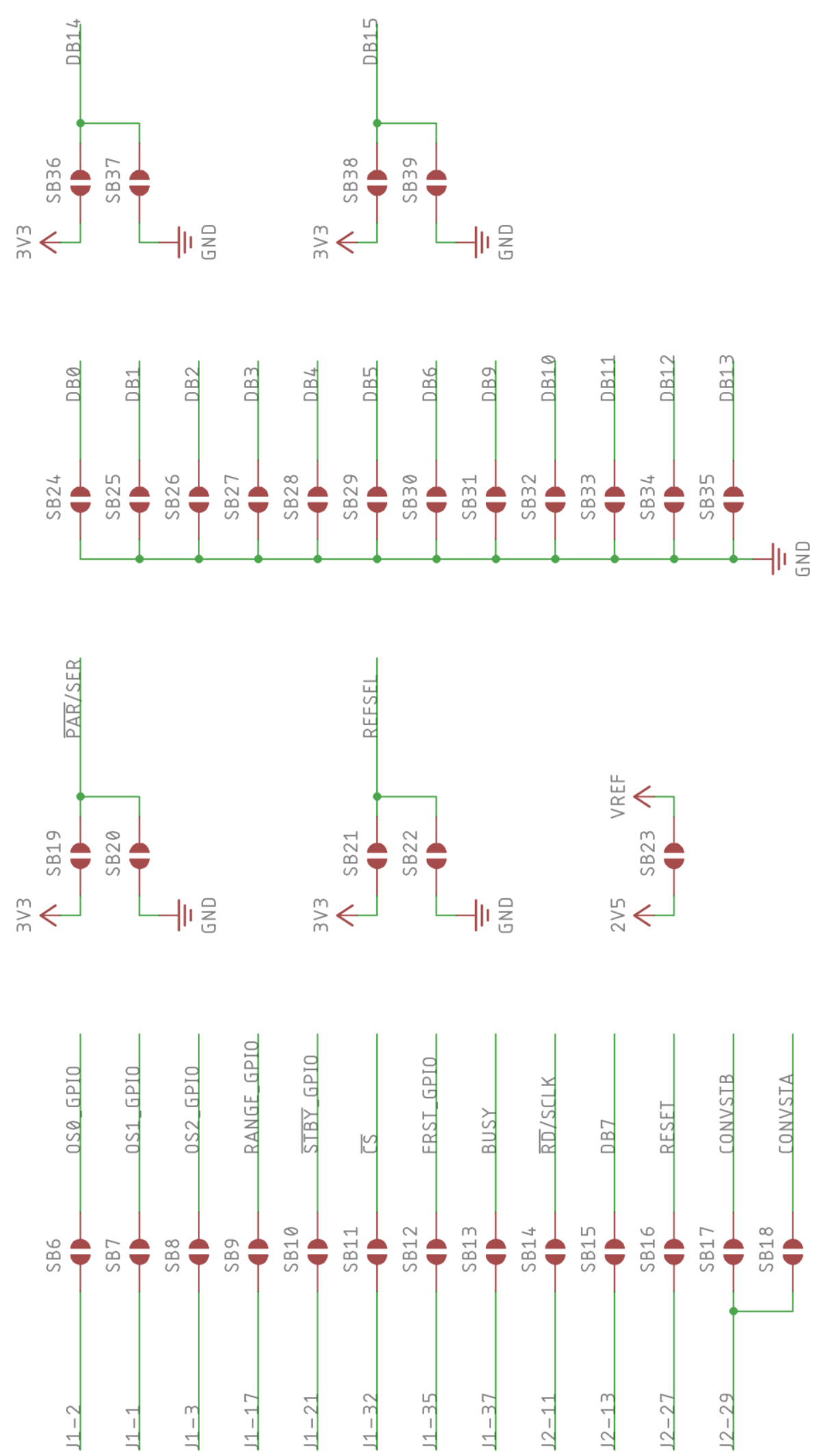

Figure A7. Schematic of the Data Acquisition Expansion Board ADC solder bridges.

69 

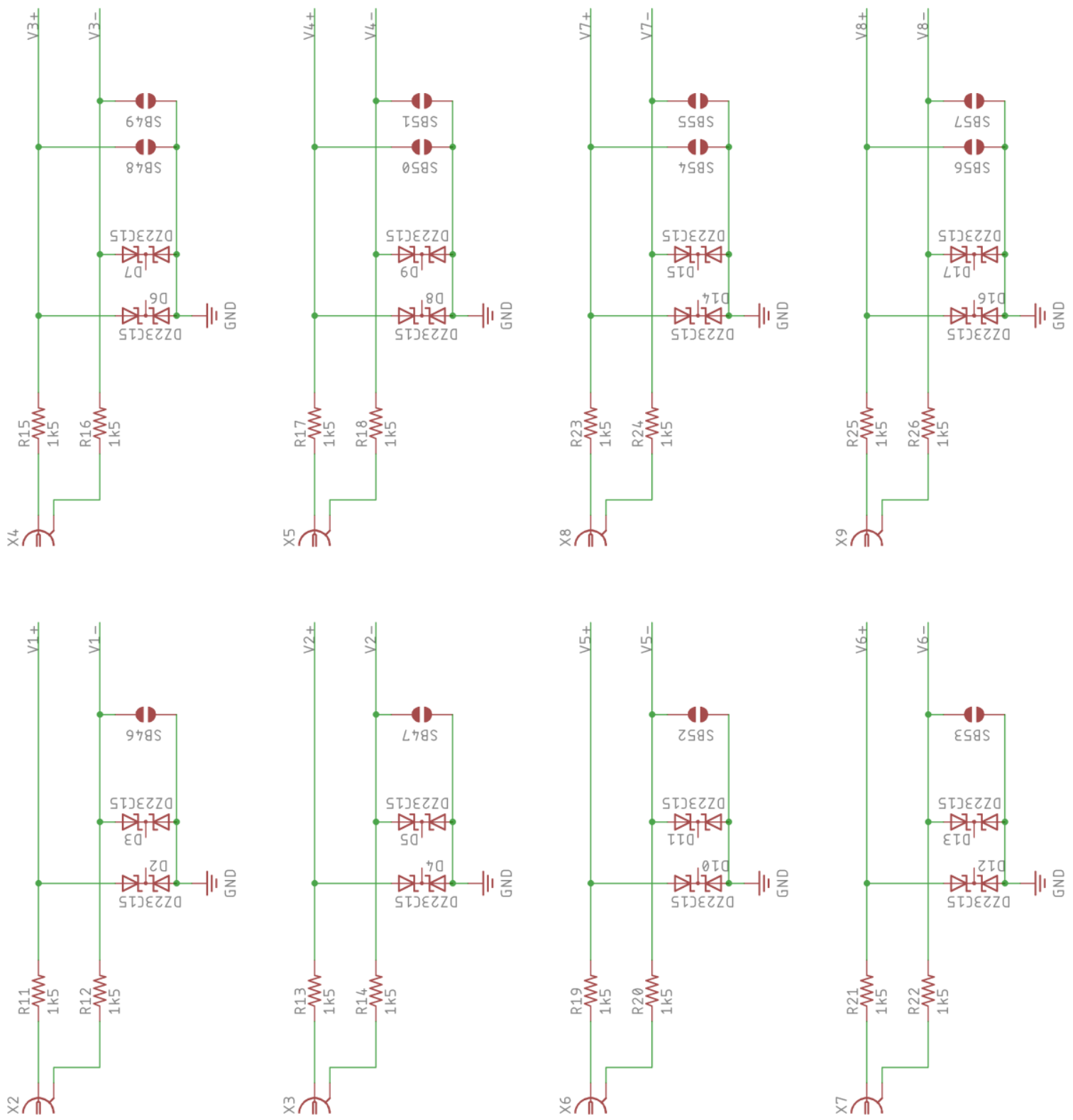

Figure A8. Schematic of the Data Acquisition Expansion Board BNC analog inputs. 


\section{Appendix B: Data Acquisition Extension Board Copper Fill}

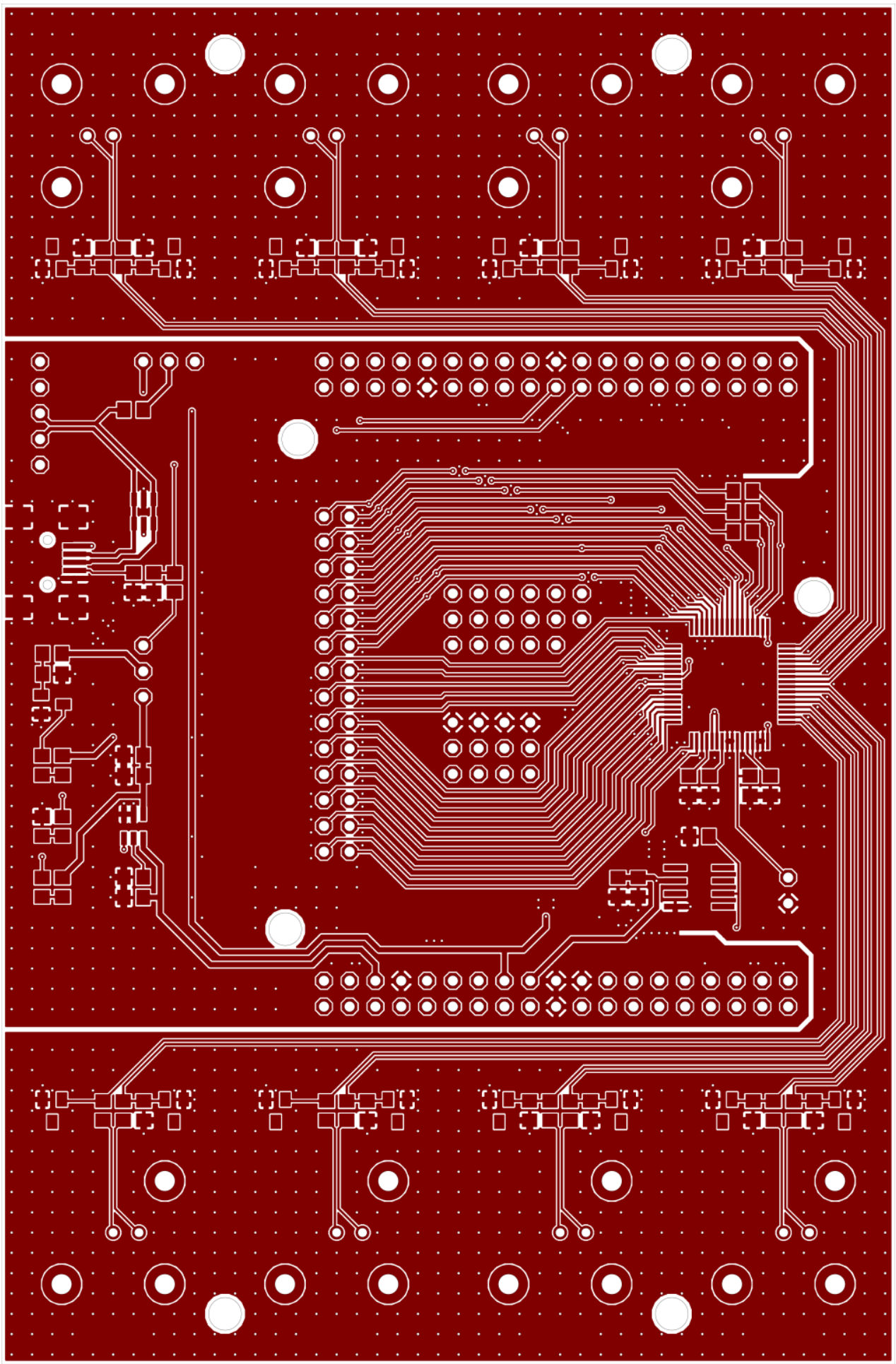

Figure B1. Top side layer copper fill of the Data Acquisition Expansion Board. 


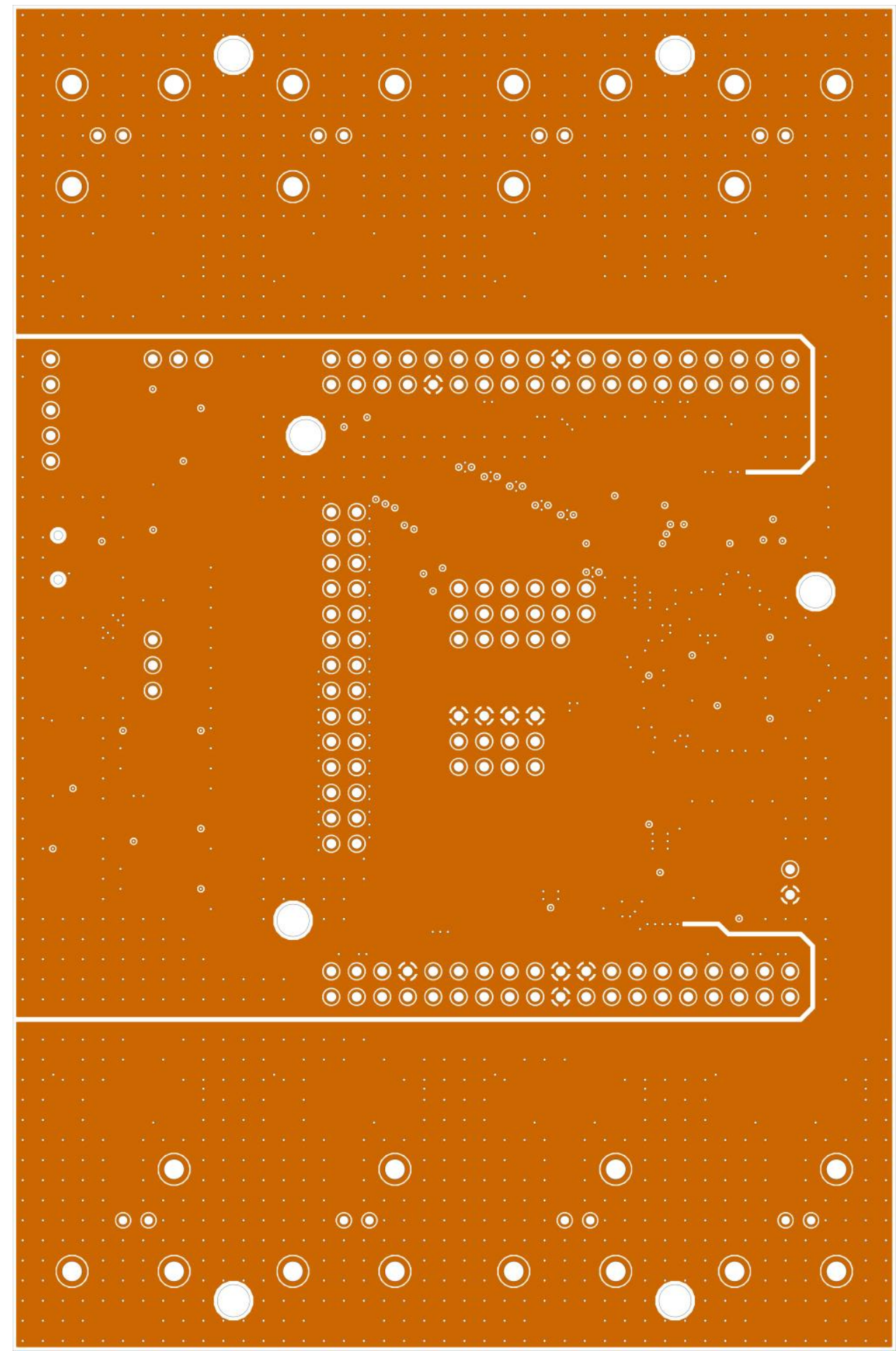

Figure B2. Top inner layer copper fill of the Data Acquisition Expansion Board. 


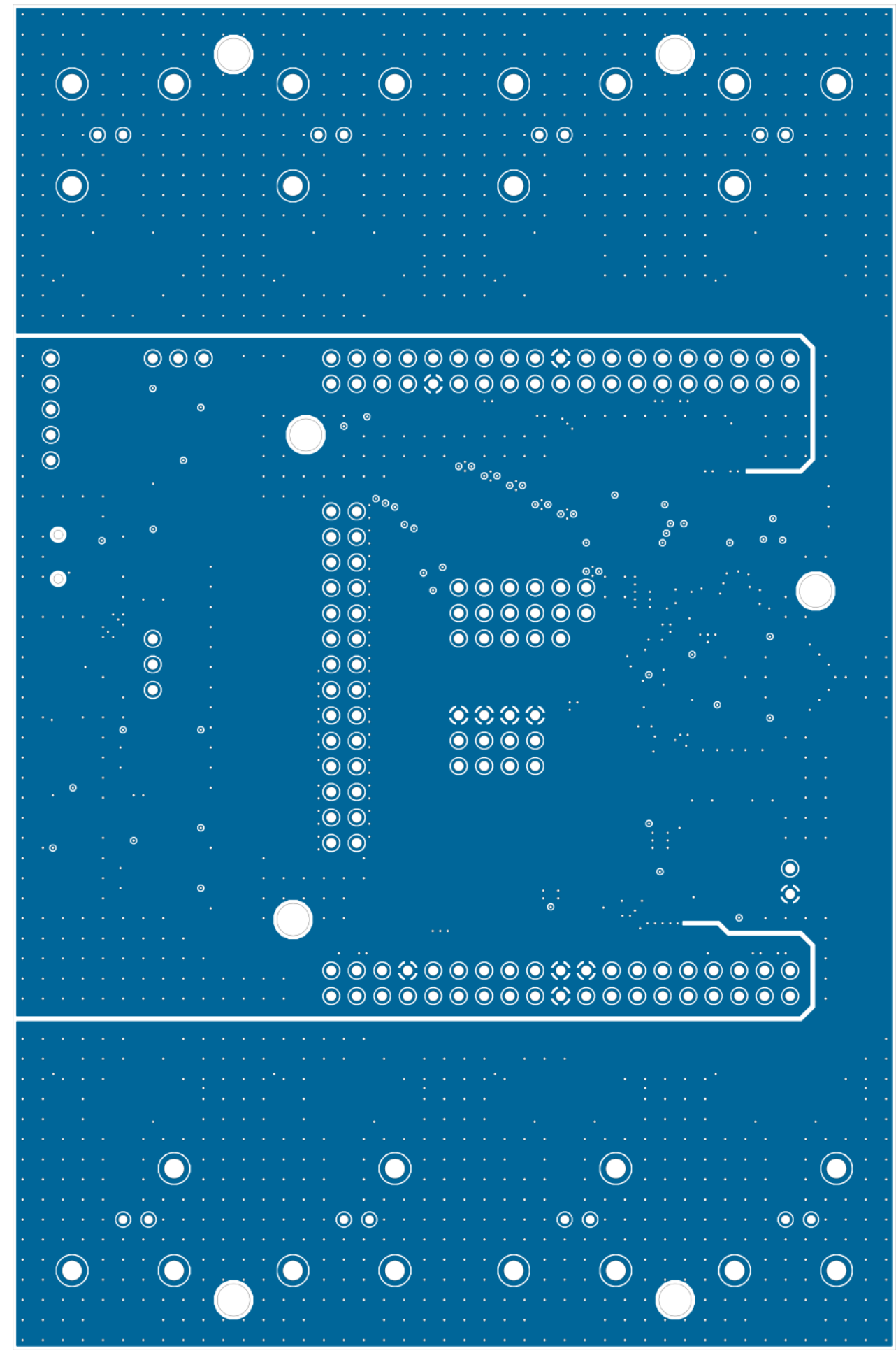

Figure B3. Bottom inner layer copper fill of the Data Acquisition Expansion Board. 


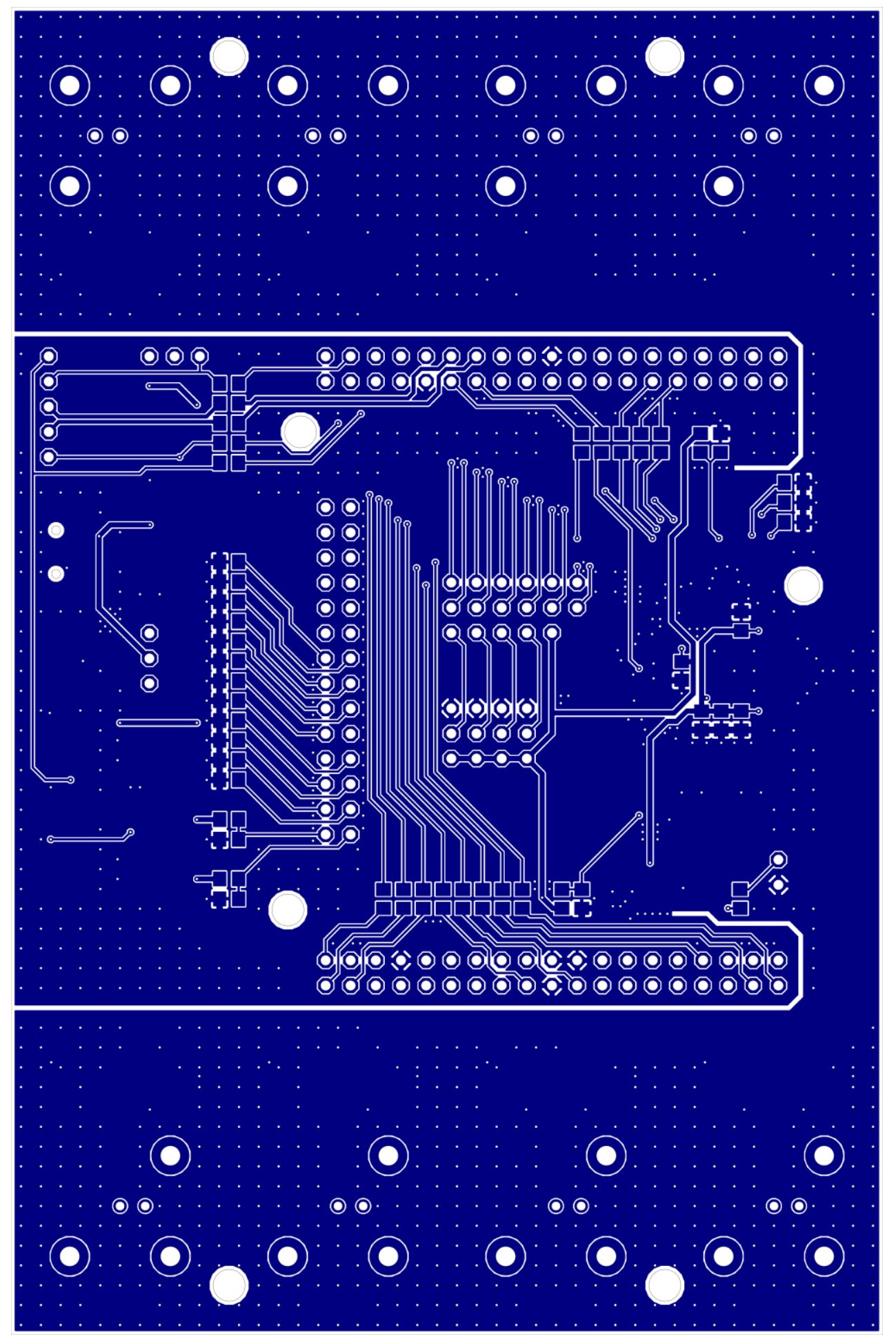

Figure B4. Bottom side layer copper fill of the Data Acquisition Expansion Board. 


\section{Appendix C: Data Acquisition Expansion Board Bill of Materials and Notes}

\begin{tabular}{|c|c|c|c|c|}
\hline Part & Value & Package & Description & Assembly Notes \\
\hline $\mathrm{C} 1$ & $10 \mu \mathrm{F}$ & $0805 / 2012$ & Capacitor & \\
\hline $\mathrm{C} 2$ & $0.1 \mu \mathrm{F}$ & $0805 / 2012$ & Capacitor & \\
\hline $\mathrm{C} 3$ & $0.1 \mu \mathrm{F}$ & $0805 / 2012$ & Capacitor & \\
\hline $\mathrm{C} 4$ & $0.1 \mu \mathrm{F}$ & $0805 / 2012$ & Capacitor & \\
\hline $\mathrm{C} 5$ & $0.1 \mu \mathrm{F}$ & $0805 / 2012$ & Capacitor & \\
\hline C6 & $0.1 \mu \mathrm{F}$ & $0805 / 2012$ & Capacitor & \\
\hline $\mathrm{C} 7$ & $0.1 \mu \mathrm{F}$ & $0805 / 2012$ & Capacitor & \\
\hline $\mathrm{C} 8$ & $0.1 \mu \mathrm{F}$ & $0805 / 2012$ & Capacitor & \\
\hline C9 & $1 \mu \mathrm{F}$ & $0805 / 2012$ & Capacitor & \\
\hline $\mathrm{C} 10$ & $1 \mu \mathrm{F}$ & $0805 / 2012$ & Capacitor & \\
\hline C11 & $0.1 \mu \mathrm{F}$ & $0805 / 2012$ & Capacitor & \\
\hline $\mathrm{C} 12$ & $10 \mu \mathrm{F}$ & $0805 / 2012$ & Capacitor & \\
\hline C13 & $10 \mu \mathrm{F}$ & $0805 / 2012$ & Capacitor & $\begin{array}{l}\text { Place if including } \\
\text { ADR421. }\end{array}$ \\
\hline $\mathrm{C} 14$ & $0.1 \mu \mathrm{F}$ & $0805 / 2012$ & Capacitor & $\begin{array}{l}\text { Place if including } \\
\text { ADR421. }\end{array}$ \\
\hline $\mathrm{C} 15$ & $0.1 \mu \mathrm{F}$ & $0805 / 2012$ & Capacitor & $\begin{array}{l}\text { Place if including } \\
\text { ADR421. }\end{array}$ \\
\hline D1 & USBLC6-2 & SOT26 & $\begin{array}{l}\text { USBLC6-2: ESD } \\
\text { Suppressor }\end{array}$ & \\
\hline D2 & DZ23C15 & SOT23 & $\begin{array}{l}\text { Dual Zener Diode: } \\
\text { Shared Cathode }\end{array}$ & \\
\hline D3 & DZ23C15 & SOT23 & $\begin{array}{l}\text { Dual Zener Diode: } \\
\text { Shared Cathode }\end{array}$ & \\
\hline D4 & DZ23C15 & SOT23 & $\begin{array}{l}\text { Dual Zener Diode: } \\
\text { Shared Cathode }\end{array}$ & \\
\hline D5 & DZ23C15 & SOT23 & $\begin{array}{l}\text { Dual Zener Diode: } \\
\text { Shared Cathode }\end{array}$ & \\
\hline D6 & DZ23C15 & SOT23 & $\begin{array}{l}\text { Dual Zener Diode: } \\
\text { Shared Cathode }\end{array}$ & $\begin{array}{l}\text { Do not place if } \\
\text { using AD7605-4 } \\
\text { or AD7606-4. }\end{array}$ \\
\hline D7 & DZ23C15 & SOT23 & $\begin{array}{l}\text { Dual Zener Diode: } \\
\text { Shared Cathode }\end{array}$ & $\begin{array}{l}\text { Do not place if } \\
\text { using AD7605-4 } \\
\text { or AD7606-4. }\end{array}$ \\
\hline D8 & DZ23C15 & SOT23 & $\begin{array}{l}\text { Dual Zener Diode: } \\
\text { Shared Cathode }\end{array}$ & $\begin{array}{l}\text { Do not place if } \\
\text { using AD7605-4, } \\
\text { AD7606-4, or } \\
\text { AD7606-6. }\end{array}$ \\
\hline D9 & DZ23C15 & SOT23 & $\begin{array}{l}\text { Dual Zener Diode: } \\
\text { Shared Cathode }\end{array}$ & $\begin{array}{l}\text { Do not place if } \\
\text { using AD7605-4, } \\
\text { AD7606-4, or } \\
\text { AD7606-6. }\end{array}$ \\
\hline
\end{tabular}




\begin{tabular}{|c|c|c|c|c|}
\hline Part & Value & Package & Description & Assembly Notes \\
\hline D10 & DZ23C15 & SOT23 & $\begin{array}{l}\text { Dual Zener Diode: } \\
\text { Shared Cathode }\end{array}$ & \\
\hline D11 & DZ23C15 & SOT23 & $\begin{array}{l}\text { Dual Zener Diode: } \\
\text { Shared Cathode }\end{array}$ & \\
\hline D12 & DZ23C15 & SOT23 & $\begin{array}{l}\text { Dual Zener Diode: } \\
\text { Shared Cathode }\end{array}$ & \\
\hline D13 & DZ23C15 & SOT23 & $\begin{array}{l}\text { Dual Zener Diode: } \\
\text { Shared Cathode }\end{array}$ & \\
\hline D14 & DZ23C15 & SOT23 & $\begin{array}{l}\text { Dual Zener Diode: } \\
\text { Shared Cathode }\end{array}$ & $\begin{array}{l}\text { Do not place if } \\
\text { using AD7605-4 } \\
\text { or AD7606-4. }\end{array}$ \\
\hline D15 & DZ23C15 & SOT23 & $\begin{array}{l}\text { Dual Zener Diode: } \\
\text { Shared Cathode }\end{array}$ & $\begin{array}{l}\text { Do not place if } \\
\text { using AD7605-4 } \\
\text { or AD7606-4. }\end{array}$ \\
\hline D16 & DZ23C15 & SOT23 & $\begin{array}{l}\text { Dual Zener Diode: } \\
\text { Shared Cathode }\end{array}$ & $\begin{array}{l}\text { Do not place if } \\
\text { using AD7605-4, } \\
\text { AD7606-4, or } \\
\text { AD7606-6. }\end{array}$ \\
\hline D17 & DZ23C15 & SOT23 & $\begin{array}{l}\text { Dual Zener Diode: } \\
\text { Shared Cathode }\end{array}$ & $\begin{array}{l}\text { Do not place if } \\
\text { using AD7605-4, } \\
\text { AD7606-4, or } \\
\text { AD7606-6. }\end{array}$ \\
\hline JP1 & & $2 \times 19,0.100 "$ & Pin Header: 2x19 & Female header. \\
\hline JP2 & & $2 \times 19,0.100 "$ & Pin Header: $2 \times 19$ & Female header. \\
\hline JP3 & & $1 \times 05,0.100 "$ & Pin Header: 1x05 & Do not place. \\
\hline JP4 & & $2 \times 14,0.100 "$ & Pin Header: 2x14 & Do not place. \\
\hline JP5 & & $1 \times 02,0.100 "$ & Pin Header: 1x02 & Do not place. \\
\hline JP6 & & $1 \times 03,0.100 "$ & Pin Header: $1 \mathrm{x} 03$ & Male header. \\
\hline JP7 & & $1 \times 03,0.100 "$ & Pin Header: 1x03 & Male header. \\
\hline JP8 & & $1 \times 03,0.100 "$ & Pin Header: 1x03 & Male header. \\
\hline JP9 & & $1 \times 03,0.100 "$ & Pin Header: 1x03 & Male header. \\
\hline JP10 & & $1 \times 03,0.100 "$ & Pin Header: 1x03 & Male header. \\
\hline JP11 & & $1 \times 03,0.100 "$ & Pin Header: 1x03 & Male header. \\
\hline JP12 & & $1 \times 03,0.100 "$ & Pin Header: 1x03 & Male header. \\
\hline JP13 & & $1 \times 03,0.100 "$ & Pin Header: 1x03 & Male header. \\
\hline JP14 & & $1 \times 03,0.100 "$ & Pin Header: 1x03 & Male header. \\
\hline JP15 & & $1 \times 03,0.100 "$ & Pin Header: 1x03 & Male header. \\
\hline JP16 & & $1 \times 03,0.100 "$ & Pin Header: 1x03 & Male header. \\
\hline JP17 & & $1 \times 02,0.100 "$ & Pin Header: 1x02 & Male header. \\
\hline LED1 & 35 mcd (Green) & $0805 / 2012$ & LED & \\
\hline LED2 & $70 \mathrm{mcd}$ (Yellow) & 0805/2012 & LED & \\
\hline LED3 & $54 \operatorname{mcd}(\operatorname{Red})$ & $0805 / 2012$ & LED & \\
\hline Q1 & BC846ALT1G & SOT23 & NPN Transistor & \\
\hline R1 & $1.5 \mathrm{k} \Omega$ & $0805 / 2012$ & Resistor & \\
\hline
\end{tabular}




\begin{tabular}{|c|c|c|c|c|}
\hline Part & Value & Package & Description & Assembly Notes \\
\hline $\mathrm{R} 2$ & $34 \mathrm{k} \Omega$ & $0805 / 2012$ & Resistor & \\
\hline R3 & $68 \mathrm{k} \Omega$ & $0805 / 2012$ & Resistor & \\
\hline $\mathrm{R} 4$ & $100 \mathrm{k} \Omega$ & $0805 / 2012$ & Resistor & \\
\hline R5 & $4.7 \mathrm{k} \Omega$ & $0805 / 2012$ & Resistor & \\
\hline R6 & $2.2 \mathrm{k} \Omega$ & $0805 / 2012$ & Resistor & \\
\hline R7 & $332 \Omega$ & $0805 / 2012$ & Resistor & \\
\hline R8 & $649 \Omega$ & $0805 / 2012$ & Resistor & \\
\hline R9 & $909 \Omega$ & $0805 / 2012$ & Resistor & \\
\hline R10 & $47.5 \mathrm{k} \Omega$ & $0805 / 2012$ & Resistor & \\
\hline R11 & $1.5 \mathrm{k} \Omega$ & $0805 / 2012$ & Resistor & \\
\hline R12 & $1.5 \mathrm{k} \Omega$ & $0805 / 2012$ & Resistor & \\
\hline R13 & $1.5 \mathrm{k} \Omega$ & $0805 / 2012$ & Resistor & \\
\hline R14 & $1.5 \mathrm{k} \Omega$ & $0805 / 2012$ & Resistor & \\
\hline R15 & $1.5 \mathrm{k} \Omega$ & $0805 / 2012$ & Resistor & $\begin{array}{l}\text { Do not place if } \\
\text { using AD7605-4 } \\
\text { or AD7606-4. }\end{array}$ \\
\hline R16 & $1.5 \mathrm{k} \Omega$ & 0805/2012 & Resistor & $\begin{array}{l}\text { Do not place if } \\
\text { using AD7605-4 } \\
\text { or AD7606-4. }\end{array}$ \\
\hline R17 & $1.5 \mathrm{k} \Omega$ & 0805/2012 & Resistor & $\begin{array}{l}\text { Do not place if } \\
\text { using AD7605-4, } \\
\text { AD7606-4, or } \\
\text { AD7606-6. }\end{array}$ \\
\hline R18 & $1.5 \mathrm{k} \Omega$ & 0805/2012 & Resistor & $\begin{array}{l}\text { Do not place if } \\
\text { using AD7605-4, } \\
\text { AD7606-4, or } \\
\text { AD7606-6. }\end{array}$ \\
\hline R19 & $1.5 \mathrm{k} \Omega$ & 0805/2012 & Resistor & \\
\hline $\mathrm{R} 20$ & $1.5 \mathrm{k} \Omega$ & $0805 / 2012$ & Resistor & \\
\hline R21 & $1.5 \mathrm{k} \Omega$ & $0805 / 2012$ & Resistor & \\
\hline R22 & $1.5 \mathrm{k} \Omega$ & $0805 / 2012$ & Resistor & \\
\hline R23 & $1.5 \mathrm{k} \Omega$ & 0805/2012 & Resistor & $\begin{array}{l}\text { Do not place if } \\
\text { using AD7605-4 } \\
\text { or AD7606-4. }\end{array}$ \\
\hline R24 & $1.5 \mathrm{k} \Omega$ & 0805/2012 & Resistor & $\begin{array}{l}\text { Do not place if } \\
\text { using AD7605-4 } \\
\text { or AD7606-4. }\end{array}$ \\
\hline R25 & $1.5 \mathrm{k} \Omega$ & 0805/2012 & Resistor & $\begin{array}{l}\text { Do not place if } \\
\text { using AD7605-4, } \\
\text { AD7606-4, or } \\
\text { AD7606-6. }\end{array}$ \\
\hline
\end{tabular}




\begin{tabular}{|c|c|c|c|c|}
\hline Part & Value & Package & Description & Assembly Notes \\
\hline R26 & $1.5 \mathrm{k} \Omega$ & $0805 / 2012$ & Resistor & $\begin{array}{l}\text { Do not place if } \\
\text { using AD7605-4, } \\
\text { AD7606-4, or } \\
\text { AD7606-6. }\end{array}$ \\
\hline SB1 & & $0805 / 2012$ & Solder Bridge & $\begin{array}{l}\text { Remove if using } \\
\text { corresponding pin } \\
\text { on JP3. }\end{array}$ \\
\hline SB2 & & $0805 / 2012$ & Solder Bridge & $\begin{array}{l}\text { Remove if using } \\
\text { corresponding pin } \\
\text { on JP3. }\end{array}$ \\
\hline SB3 & & 0805/2012 & Solder Bridge & $\begin{array}{l}\text { Remove if using } \\
\text { corresponding pin } \\
\text { on JP3. }\end{array}$ \\
\hline SB4 & & 0805/2012 & Solder Bridge & $\begin{array}{l}\text { Remove if using } \\
\text { corresponding pin } \\
\text { on JP3. }\end{array}$ \\
\hline SB5 & & $0805 / 2012$ & Solder Bridge & $\begin{array}{l}\text { Remove if using } \\
\text { corresponding pin } \\
\text { on JP3. }\end{array}$ \\
\hline SB6 & & $0805 / 2012$ & Solder Bridge & $\begin{array}{l}\text { Do not place if } \\
\text { using AD7605-4. } \\
\text { Remove if using } \\
\text { corresponding pin } \\
\text { on JP4. }\end{array}$ \\
\hline SB7 & & 0805/2012 & Solder Bridge & $\begin{array}{l}\text { Do not place if } \\
\text { using AD7605-4. } \\
\text { Remove if using } \\
\text { corresponding pin } \\
\text { on JP4. }\end{array}$ \\
\hline SB8 & & 0805/2012 & Solder Bridge & $\begin{array}{l}\text { Do not place if } \\
\text { using AD7605-4. } \\
\text { Remove if using } \\
\text { corresponding pin } \\
\text { on JP4. }\end{array}$ \\
\hline SB9 & & 0805/2012 & Solder Bridge & $\begin{array}{l}\text { Remove if using } \\
\text { corresponding pin } \\
\text { on JP4. }\end{array}$ \\
\hline SB10 & & $0805 / 2012$ & Solder Bridge & $\begin{array}{l}\text { Remove if using } \\
\text { corresponding pin } \\
\text { on JP4. }\end{array}$ \\
\hline SB11 & & 0805/2012 & Solder Bridge & $\begin{array}{l}\text { Remove if using } \\
\text { corresponding pin } \\
\text { on JP4. }\end{array}$ \\
\hline
\end{tabular}




\begin{tabular}{|c|c|c|c|c|}
\hline Part & Value & Package & Description & Assembly Notes \\
\hline SB12 & & 0805/2012 & Solder Bridge & $\begin{array}{l}\text { Remove if using } \\
\text { corresponding pin } \\
\text { on JP4. }\end{array}$ \\
\hline SB13 & & $0805 / 2012$ & Solder Bridge & $\begin{array}{l}\text { Remove if using } \\
\text { corresponding pin } \\
\text { on JP4. }\end{array}$ \\
\hline SB14 & & $0805 / 2012$ & Solder Bridge & $\begin{array}{l}\text { Remove if using } \\
\text { corresponding pin } \\
\text { on JP4. }\end{array}$ \\
\hline SB15 & & 0805/2012 & Solder Bridge & $\begin{array}{l}\text { Remove if using } \\
\text { corresponding pin } \\
\text { on JP4. }\end{array}$ \\
\hline SB16 & & $0805 / 2012$ & Solder Bridge & $\begin{array}{l}\text { Remove if using } \\
\text { corresponding pin } \\
\text { on JP4. }\end{array}$ \\
\hline SB17 & & 0805/2012 & Solder Bridge & $\begin{array}{l}\text { Remove if using } \\
\text { corresponding pin } \\
\text { on JP4. }\end{array}$ \\
\hline SB18 & & 0805/2012 & Solder Bridge & $\begin{array}{l}\text { Remove if using } \\
\text { corresponding pin } \\
\text { on JP4. }\end{array}$ \\
\hline SB19 & & 0805/2012 & Solder Bridge & $\begin{array}{l}\text { Place if using } \\
\text { serial or parallel } \\
\text { byte mode. SB20 } \\
\text { must be removed. }\end{array}$ \\
\hline SB20 & & 0805/2012 & Solder Bridge & $\begin{array}{l}\text { Place if using } \\
\text { parallel mode. } \\
\text { SB19 must be } \\
\text { removed. }\end{array}$ \\
\hline SB21 & & 0805/2012 & Solder Bridge & $\begin{array}{l}\text { Place if using } \\
\text { internal reference. } \\
\text { SB22 must be } \\
\text { removed. }\end{array}$ \\
\hline SB22 & & $0805 / 2012$ & Solder Bridge & $\begin{array}{l}\text { Place if using } \\
\text { external } \\
\text { reference. SB21 } \\
\text { must be removed. }\end{array}$ \\
\hline SB23 & & 0805/2012 & Solder Bridge & $\begin{array}{l}\text { Place if using on- } \\
\text { board external } \\
\text { reference. }\end{array}$ \\
\hline SB24 & & 0805/2012 & Solder Bridge & $\begin{array}{l}\text { Place if using } \\
\text { serial mode. }\end{array}$ \\
\hline SB25 & & $0805 / 2012$ & Solder Bridge & $\begin{array}{l}\text { Place if using } \\
\text { serial mode. }\end{array}$ \\
\hline
\end{tabular}




\begin{tabular}{|c|c|c|c|c|}
\hline Part & Value & Package & Description & Assembly Notes \\
\hline SB26 & & $0805 / 2012$ & Solder Bridge & $\begin{array}{l}\text { Place if using } \\
\text { serial mode. }\end{array}$ \\
\hline SB27 & & 0805/2012 & Solder Bridge & $\begin{array}{l}\text { Place if using } \\
\text { serial mode. }\end{array}$ \\
\hline SB28 & & 0805/2012 & Solder Bridge & $\begin{array}{l}\text { Place if using } \\
\text { serial mode. }\end{array}$ \\
\hline SB29 & & 0805/2012 & Solder Bridge & $\begin{array}{l}\text { Place if using } \\
\text { serial mode. }\end{array}$ \\
\hline SB30 & & $0805 / 2012$ & Solder Bridge & $\begin{array}{l}\text { Place if using } \\
\text { serial mode. }\end{array}$ \\
\hline SB31 & & 0805/2012 & Solder Bridge & $\begin{array}{l}\text { Place if using } \\
\text { serial or parallel } \\
\text { byte mode. }\end{array}$ \\
\hline SB32 & & 0805/2012 & Solder Bridge & $\begin{array}{l}\text { Place if using } \\
\text { serial or parallel } \\
\text { byte mode. }\end{array}$ \\
\hline SB33 & & 0805/2012 & Solder Bridge & $\begin{array}{l}\text { Place if using } \\
\text { serial or parallel } \\
\text { byte mode. }\end{array}$ \\
\hline SB34 & & $0805 / 2012$ & Solder Bridge & $\begin{array}{l}\text { Place if using } \\
\text { serial or parallel } \\
\text { byte mode. }\end{array}$ \\
\hline SB35 & & 0805/2012 & Solder Bridge & $\begin{array}{l}\text { Place if using } \\
\text { serial or parallel } \\
\text { byte mode. }\end{array}$ \\
\hline SB36 & & 0805/2012 & Solder Bridge & $\begin{array}{l}\text { Place if using } \\
\text { parallel byte } \\
\text { mode with MSB } \\
\text { clocked out first. } \\
\text { Not applicable for } \\
\text { AD7608 and } \\
\text { AD7609. }\end{array}$ \\
\hline SB37 & & $0805 / 2012$ & Solder Bridge & $\begin{array}{l}\text { Place if using } \\
\text { serial mode or if } \\
\text { using parallel } \\
\text { byte mode with } \\
\text { LSB clocked out } \\
\text { first. }\end{array}$ \\
\hline SB38 & & 0805/2012 & Solder Bridge & $\begin{array}{l}\text { Place if using } \\
\text { parallel byte } \\
\text { mode. Not } \\
\text { applicable for } \\
\text { AD7608 and } \\
\text { AD7609. }\end{array}$ \\
\hline
\end{tabular}




\begin{tabular}{|c|c|c|c|c|}
\hline Part & Value & Package & Description & Assembly Notes \\
\hline SB39 & & $0805 / 2012$ & Solder Bridge & $\begin{array}{l}\text { Place if using } \\
\text { serial mode. }\end{array}$ \\
\hline SB40 & & 0805/2012 & Solder Bridge & $\begin{array}{l}\text { Do not place if } \\
\text { using AD7605-4. }\end{array}$ \\
\hline SB41 & & $0805 / 2012$ & Solder Bridge & $\begin{array}{l}\text { Do not place if } \\
\text { using AD7605-4. }\end{array}$ \\
\hline SB42 & & 0805/2012 & Solder Bridge & $\begin{array}{l}\text { Do not place if } \\
\text { using AD7605-4. }\end{array}$ \\
\hline SB43 & & $0805 / 2012$ & Solder Bridge & $\begin{array}{l}\text { Place if using } \\
\text { AD7605-4. }\end{array}$ \\
\hline SB44 & & $0805 / 2012$ & Solder Bridge & $\begin{array}{l}\text { Place if using } \\
\text { AD7605-4. }\end{array}$ \\
\hline SB45 & & 0805/2012 & Solder Bridge & $\begin{array}{l}\text { Place if using } \\
\text { AD7605-4. }\end{array}$ \\
\hline SB46 & & $0805 / 2012$ & Solder Bridge & $\begin{array}{l}\text { Do not place if } \\
\text { using AD7609. }\end{array}$ \\
\hline SB47 & & 0805/2012 & Solder Bridge & $\begin{array}{l}\text { Do not place if } \\
\text { using AD7609. }\end{array}$ \\
\hline SB48 & & $0805 / 2012$ & Solder Bridge & $\begin{array}{l}\text { Place if using } \\
\text { AD7605-4 or } \\
\text { AD7606-4. }\end{array}$ \\
\hline SB49 & & $0805 / 2012$ & Solder Bridge & $\begin{array}{l}\text { Do not place if } \\
\text { using AD7609. }\end{array}$ \\
\hline SB50 & & 0805/2012 & Solder Bridge & $\begin{array}{l}\text { Place if using } \\
\text { AD7605-4, } \\
\text { AD7606-4, or } \\
\text { AD7606-6. }\end{array}$ \\
\hline SB51 & & $0805 / 2012$ & Solder Bridge & $\begin{array}{l}\text { Do not place if } \\
\text { using AD7609. }\end{array}$ \\
\hline SB52 & & $0805 / 2012$ & Solder Bridge & $\begin{array}{l}\text { Do not place if } \\
\text { using AD7609. }\end{array}$ \\
\hline SB53 & & 0805/2012 & Solder Bridge & $\begin{array}{l}\text { Do not place if } \\
\text { using AD7609. }\end{array}$ \\
\hline SB54 & & 0805/2012 & Solder Bridge & $\begin{array}{l}\text { Place if using } \\
\text { AD7605-4 or } \\
\text { AD7606-4. }\end{array}$ \\
\hline SB55 & & $0805 / 2012$ & Solder Bridge & $\begin{array}{l}\text { Do not place if } \\
\text { using AD7609. }\end{array}$ \\
\hline SB56 & & 0805/2012 & Solder Bridge & $\begin{array}{l}\text { Place if using } \\
\text { AD7605-4, } \\
\text { AD7606-4, or } \\
\text { AD7606-6. }\end{array}$ \\
\hline SB57 & & $0805 / 2012$ & Solder Bridge & $\begin{array}{l}\text { Do not place if } \\
\text { using AD7609. }\end{array}$ \\
\hline
\end{tabular}




\begin{tabular}{|c|c|c|c|c|}
\hline Part & Value & Package & Description & Assembly Notes \\
\hline $\mathrm{U} 1$ & AP2552 & SOT26 & $\begin{array}{l}\text { AP255X: Current- } \\
\text { Limited Switch }\end{array}$ & \\
\hline U2 & AD760X* & LQFP64 & AD760X: ADC & $\begin{array}{l}\text { See Table } 2 \text { for a } \\
\text { list of compatible } \\
\text { ADCs. }\end{array}$ \\
\hline U3 & ADR421 & SOIC-8 & $\begin{array}{l}\text { ADR42X: Voltage } \\
\text { Reference }\end{array}$ & Optional. \\
\hline $\mathrm{X} 1$ & & USB Mini: 5-Pin & USB Mini-B: 5-Pin & \\
\hline $\mathrm{X} 2$ & & BNC - Isolated & BNC - Isolated & \\
\hline $\mathrm{X} 3$ & & BNC - Isolated & BNC - Isolated & \\
\hline $\mathrm{X} 4$ & & BNC - Isolated & BNC - Isolated & $\begin{array}{l}\text { Do not place if } \\
\text { using AD7605-4 } \\
\text { or AD7606-4. }\end{array}$ \\
\hline $\mathrm{X} 5$ & & BNC - Isolated & BNC - Isolated & $\begin{array}{l}\text { Do not place if } \\
\text { using AD7605-4, } \\
\text { AD7606-4, or } \\
\text { AD7606-6. }\end{array}$ \\
\hline $\mathrm{X} 6$ & & BNC - Isolated & BNC - Isolated & \\
\hline $\mathrm{X} 7$ & & BNC - Isolated & BNC - Isolated & \\
\hline X8 & & BNC - Isolated & BNC - Isolated & $\begin{array}{l}\text { Do not place if } \\
\text { using AD7605-4 } \\
\text { or AD7606-4. }\end{array}$ \\
\hline X9 & & BNC - Isolated & BNC - Isolated & $\begin{array}{l}\text { Do not place if } \\
\text { using AD7605-4, } \\
\text { AD7606-4, or } \\
\text { AD7606-6. }\end{array}$ \\
\hline
\end{tabular}




\section{Appendix D: STM32 Device Library APIs}

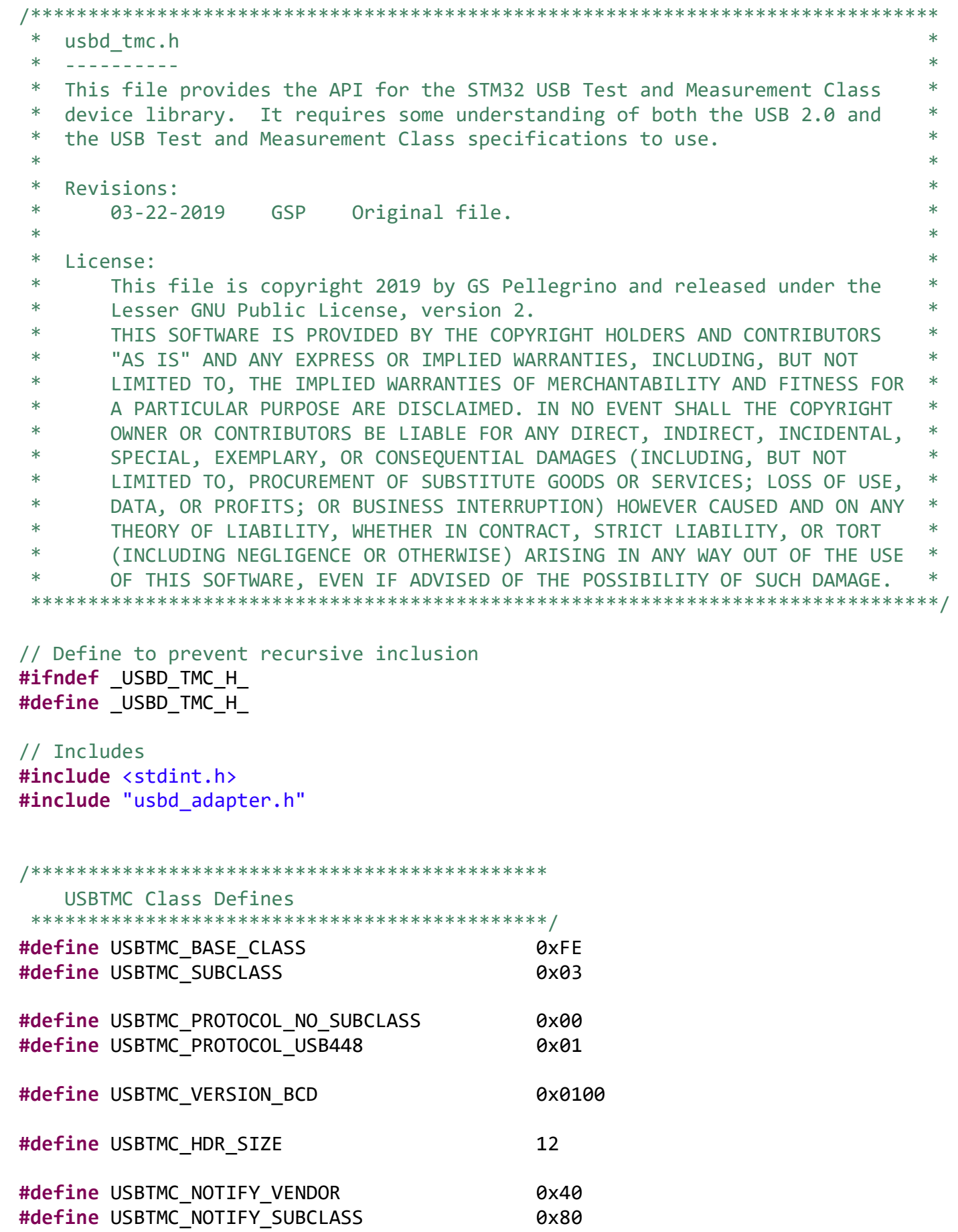




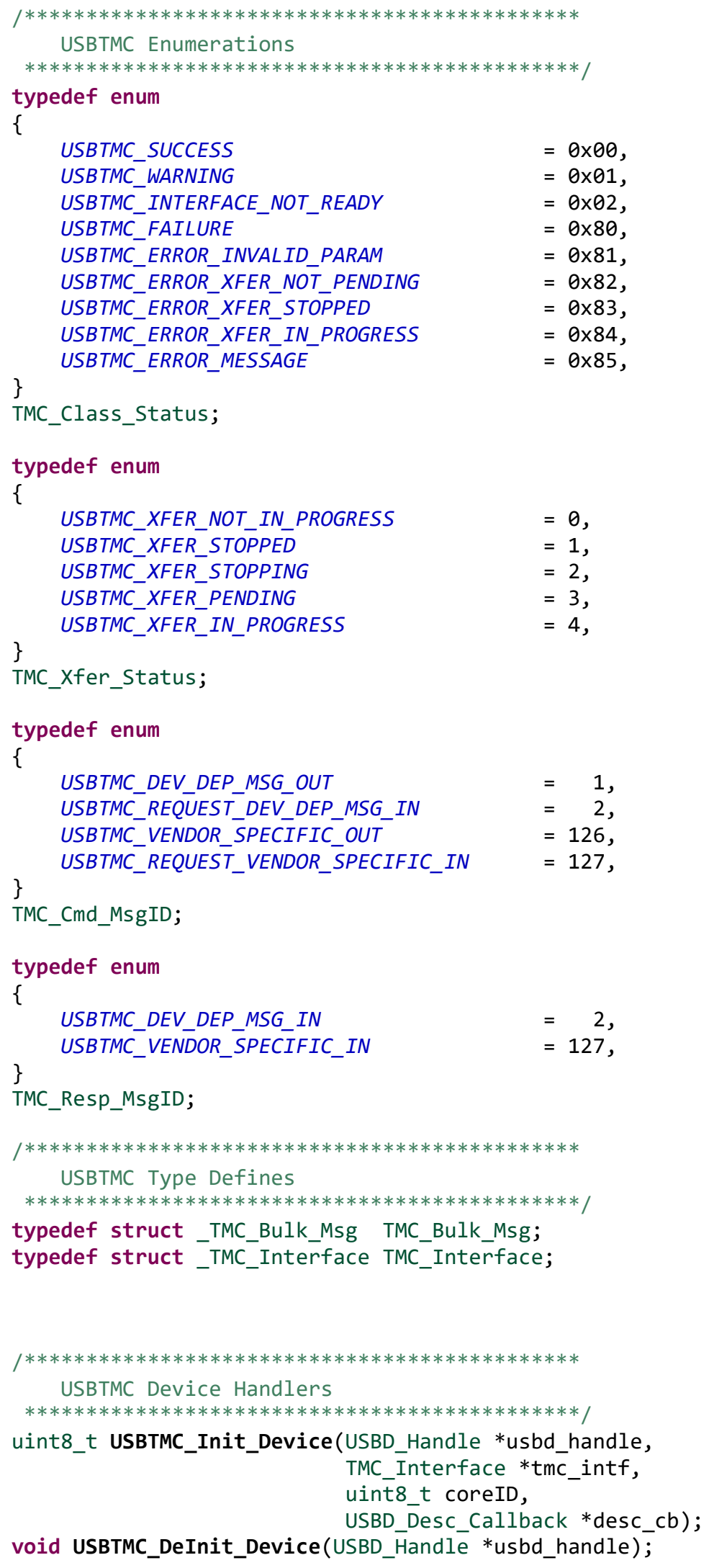




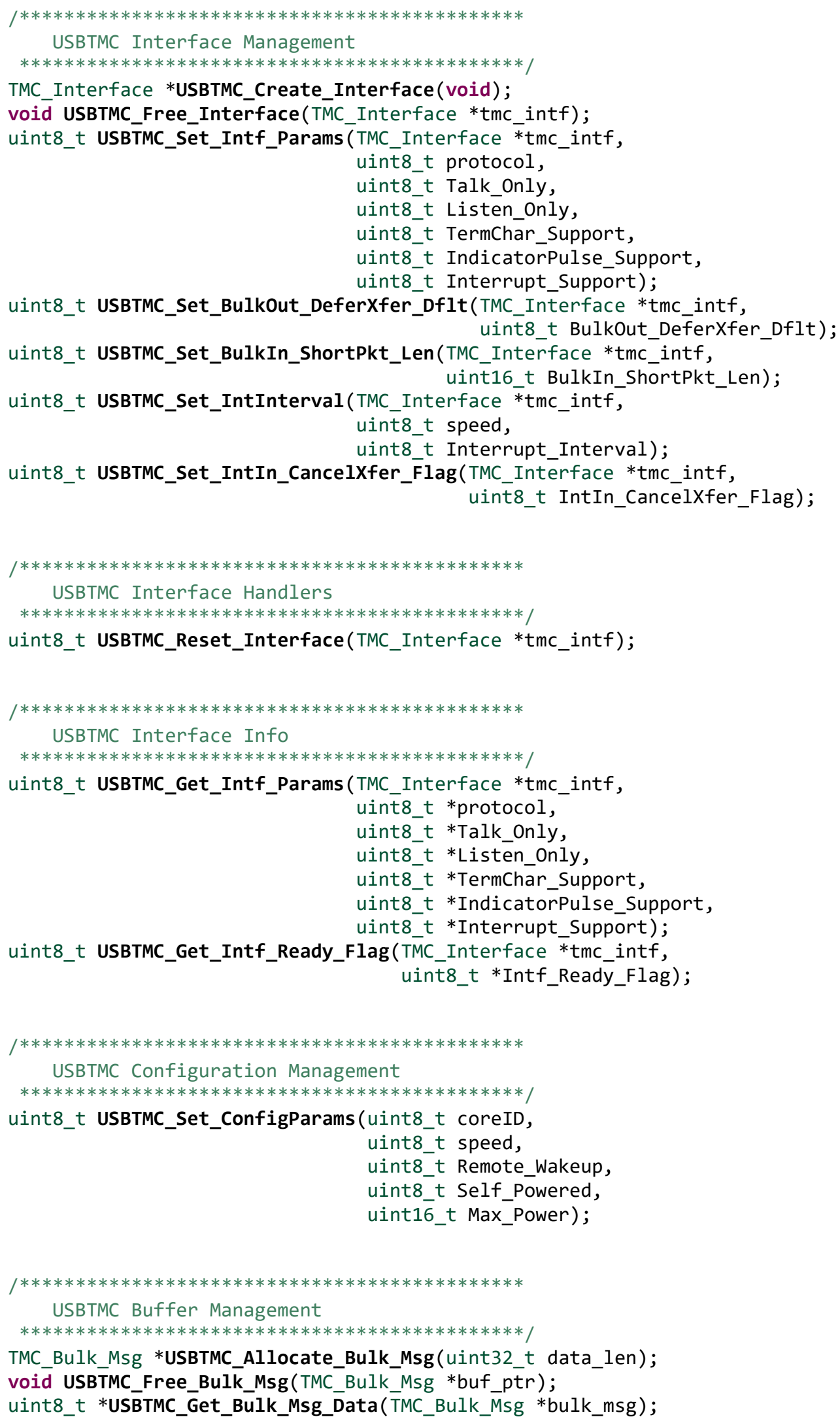




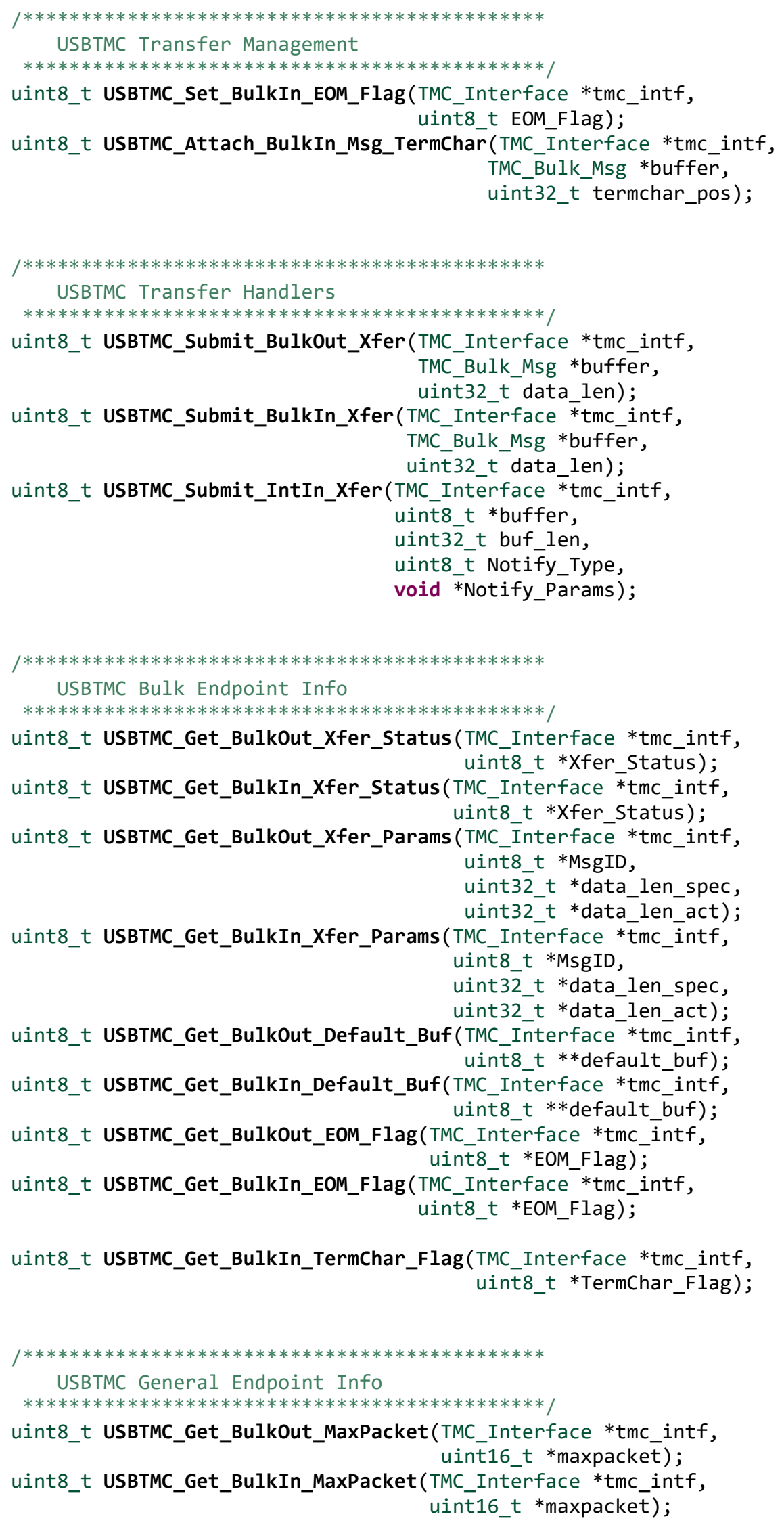


uint8_t USBTMC_Get_IntIn_MaxPacket(TMC_Interface *tmc_intf, uint16_t *maxpacket);

\#endif /* USBD_TMC_H_ $* /$ 


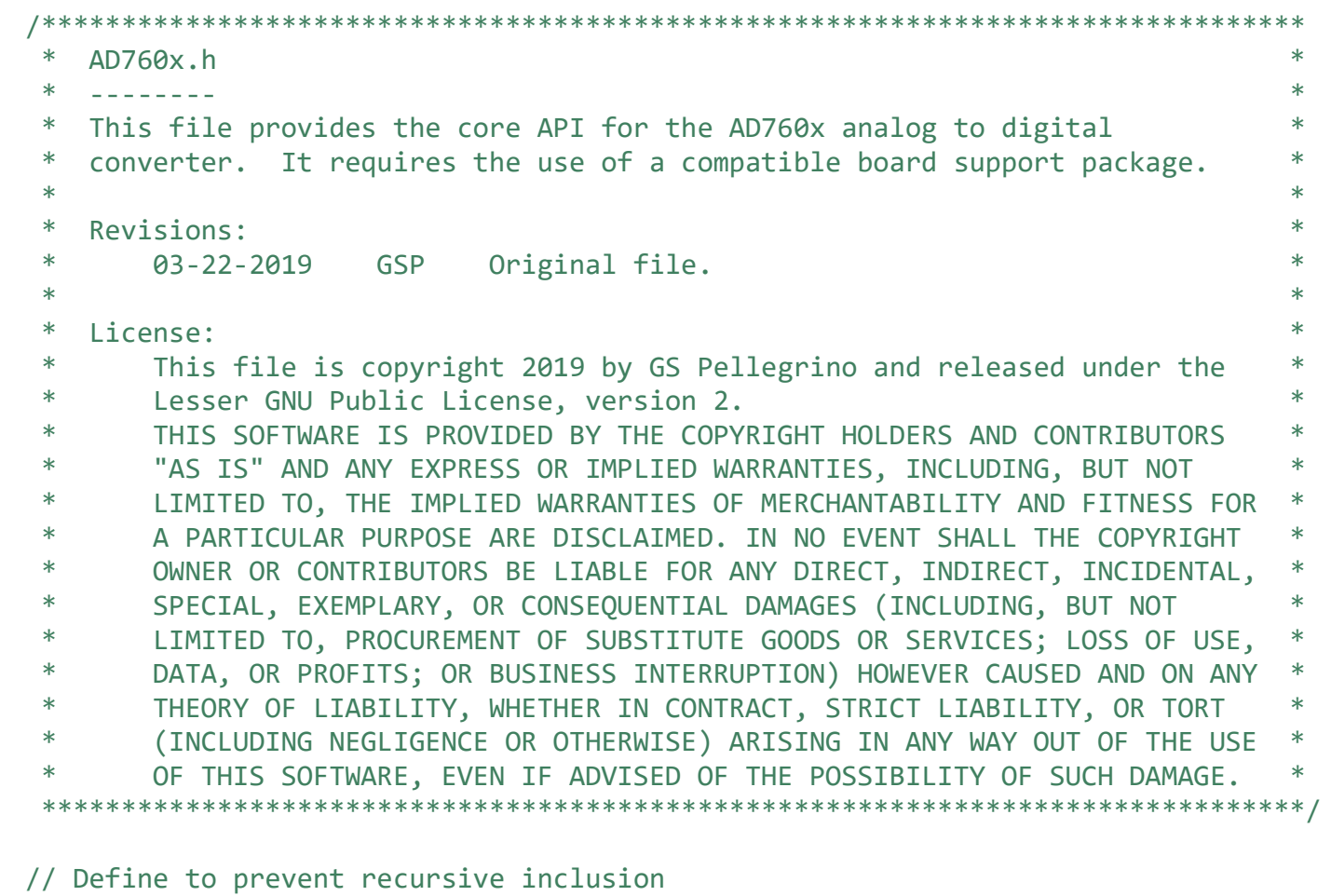

\#ifndef_AD760X_H

\#define _AD760X_H_

// Includes

\#include <stdint.h〉

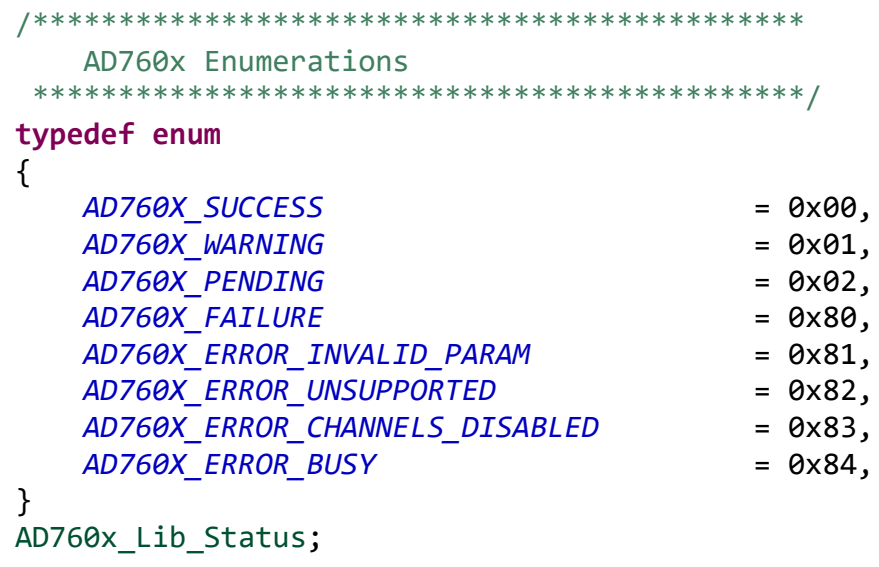




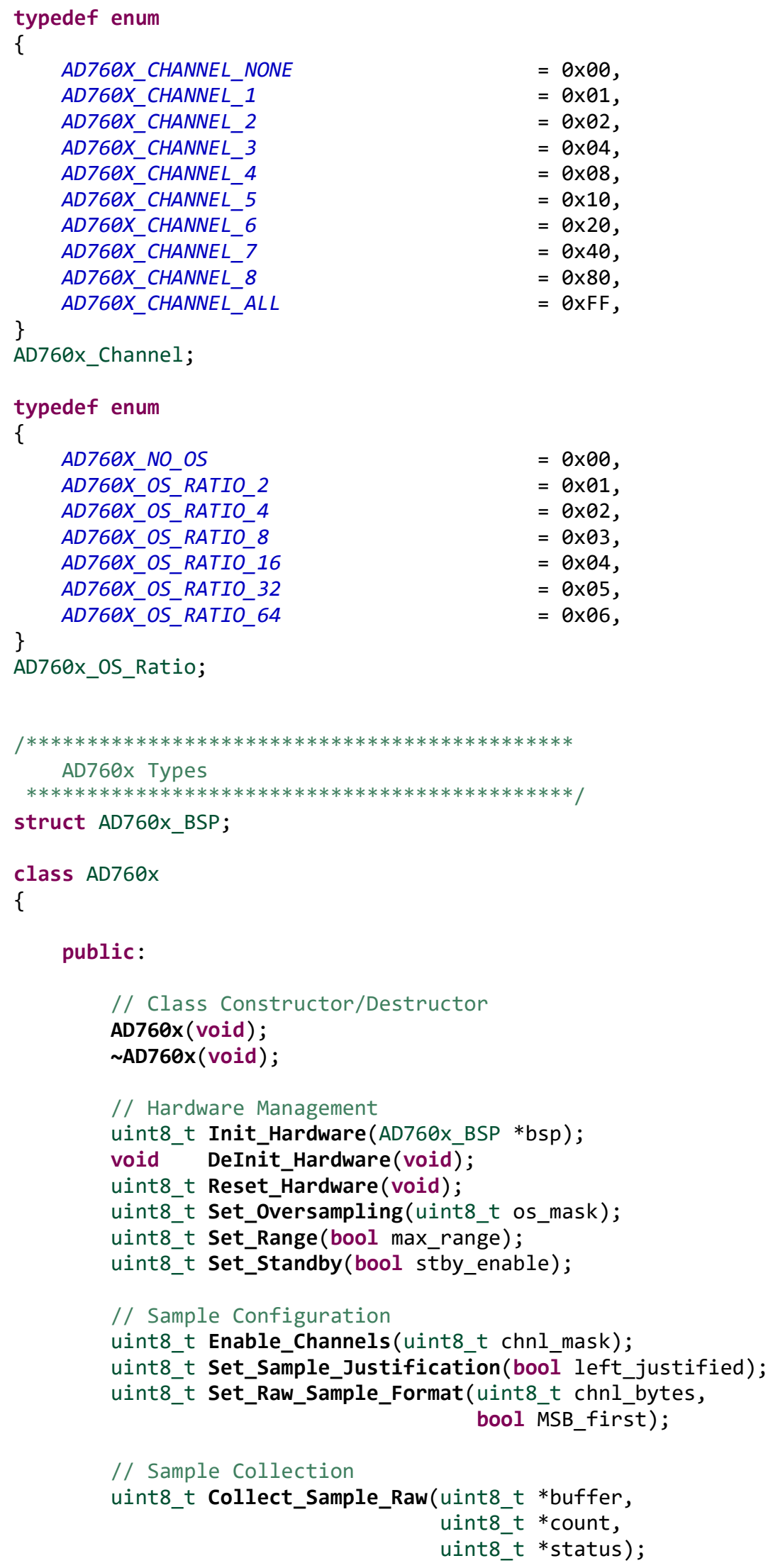




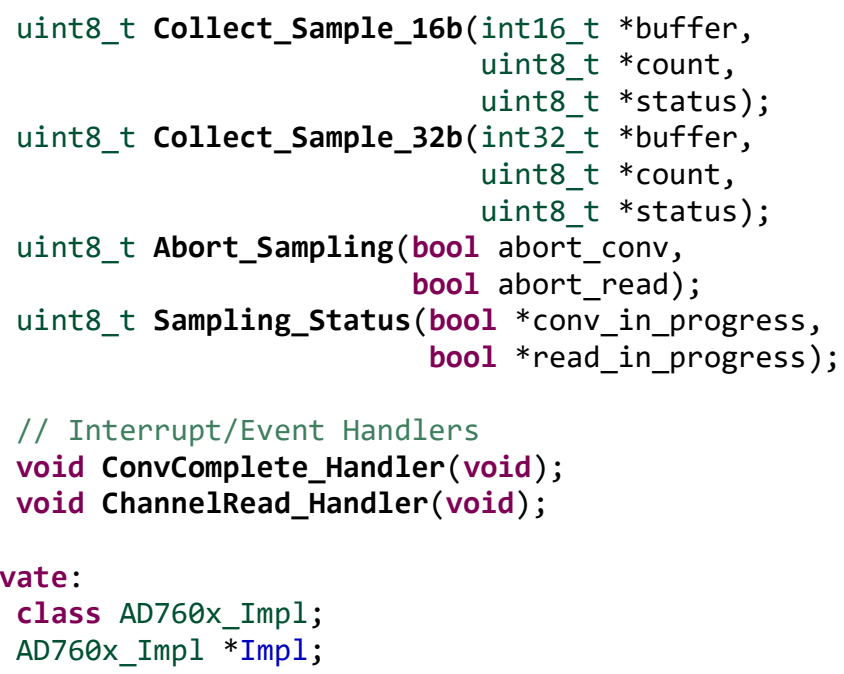



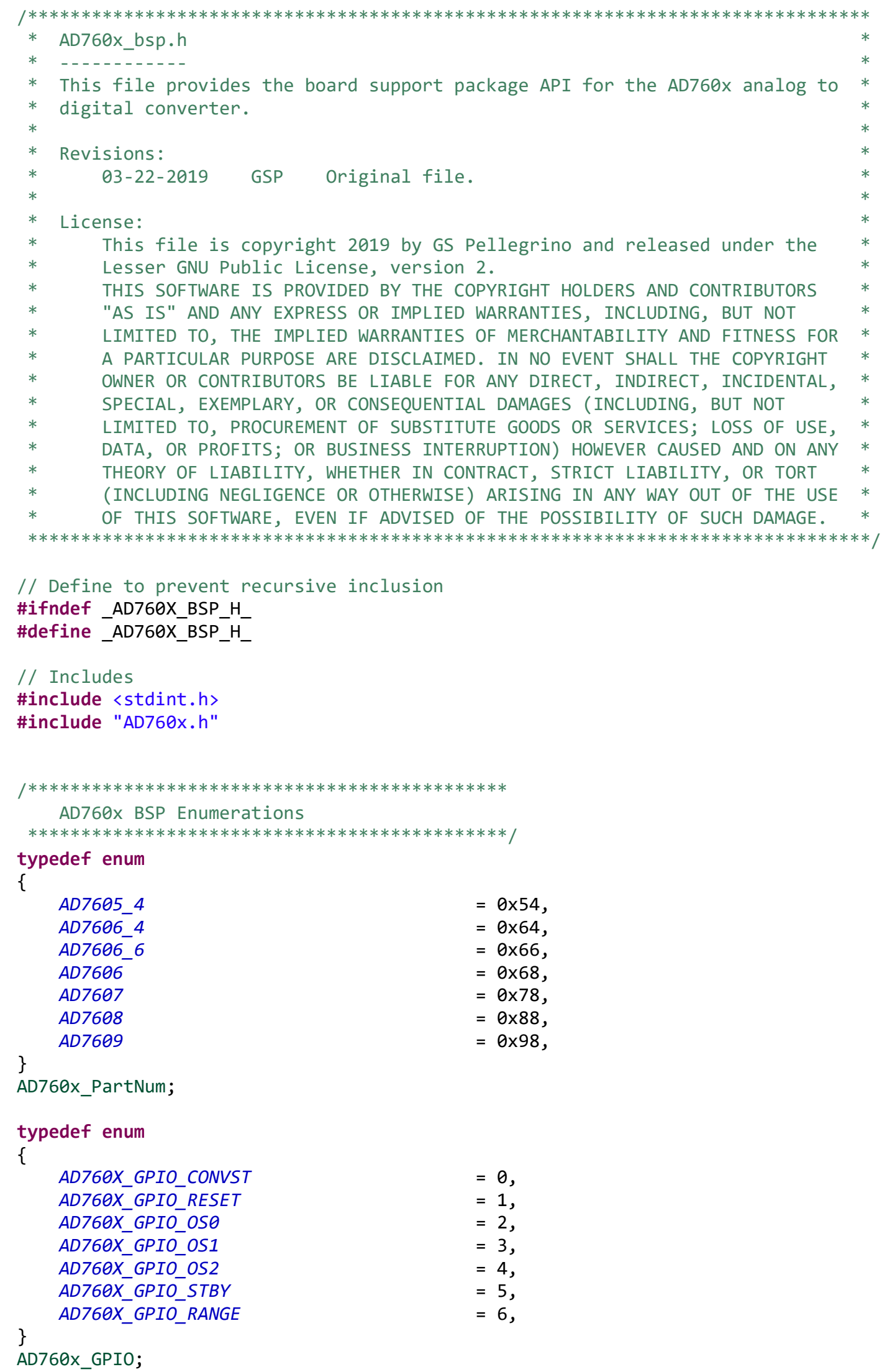


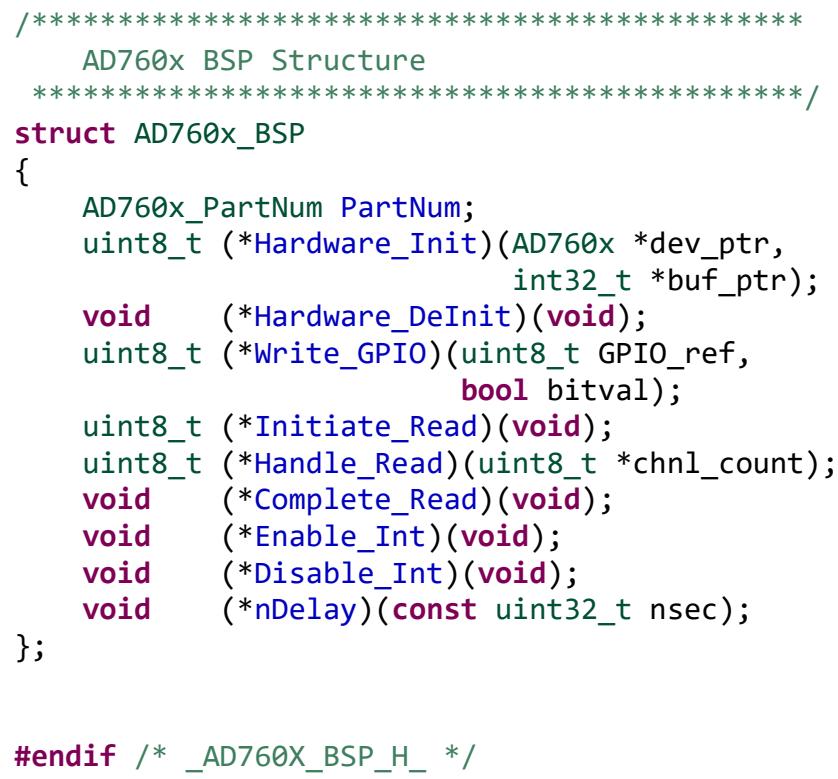




\section{Appendix E: Computer Library APIs}

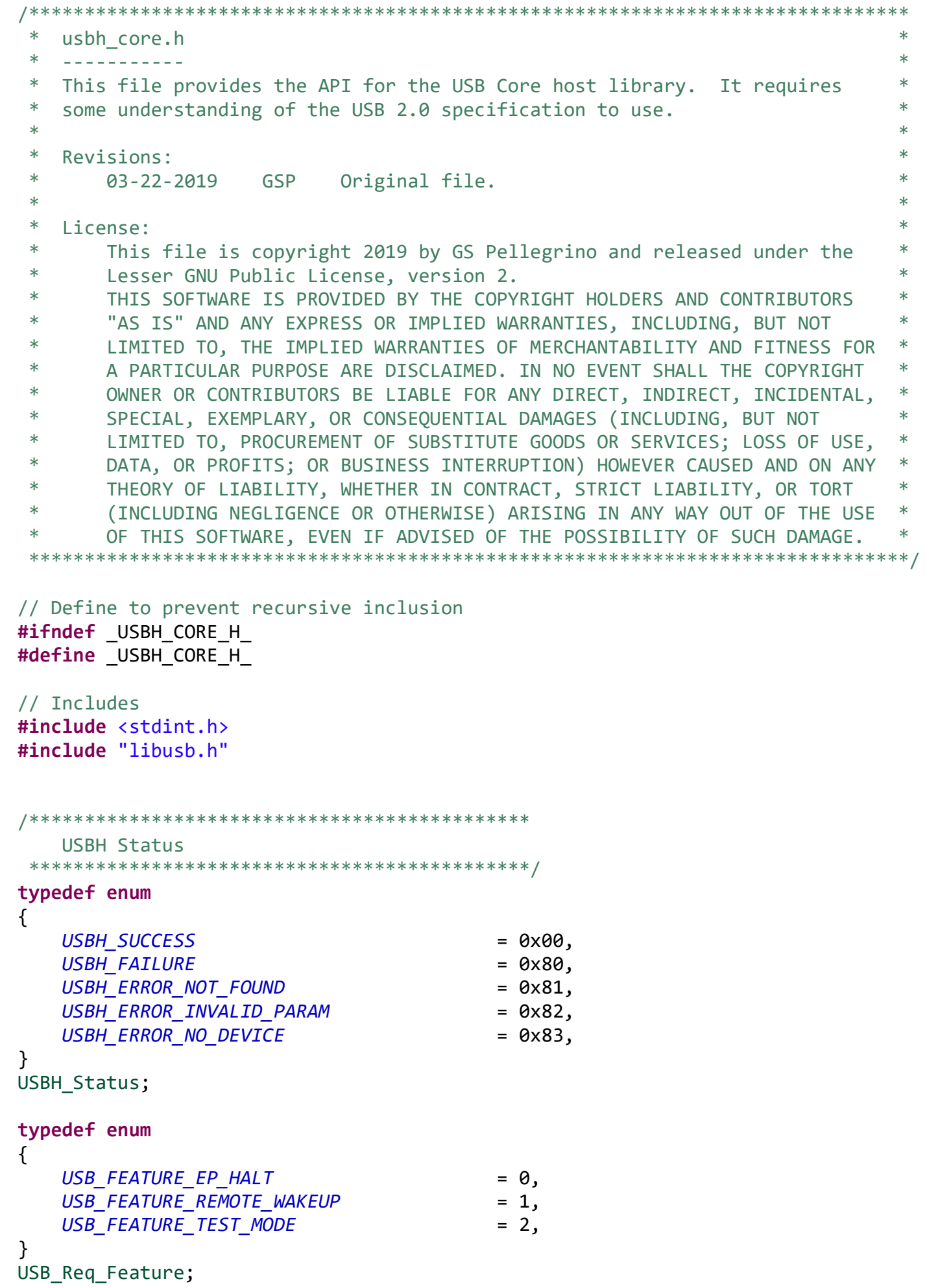




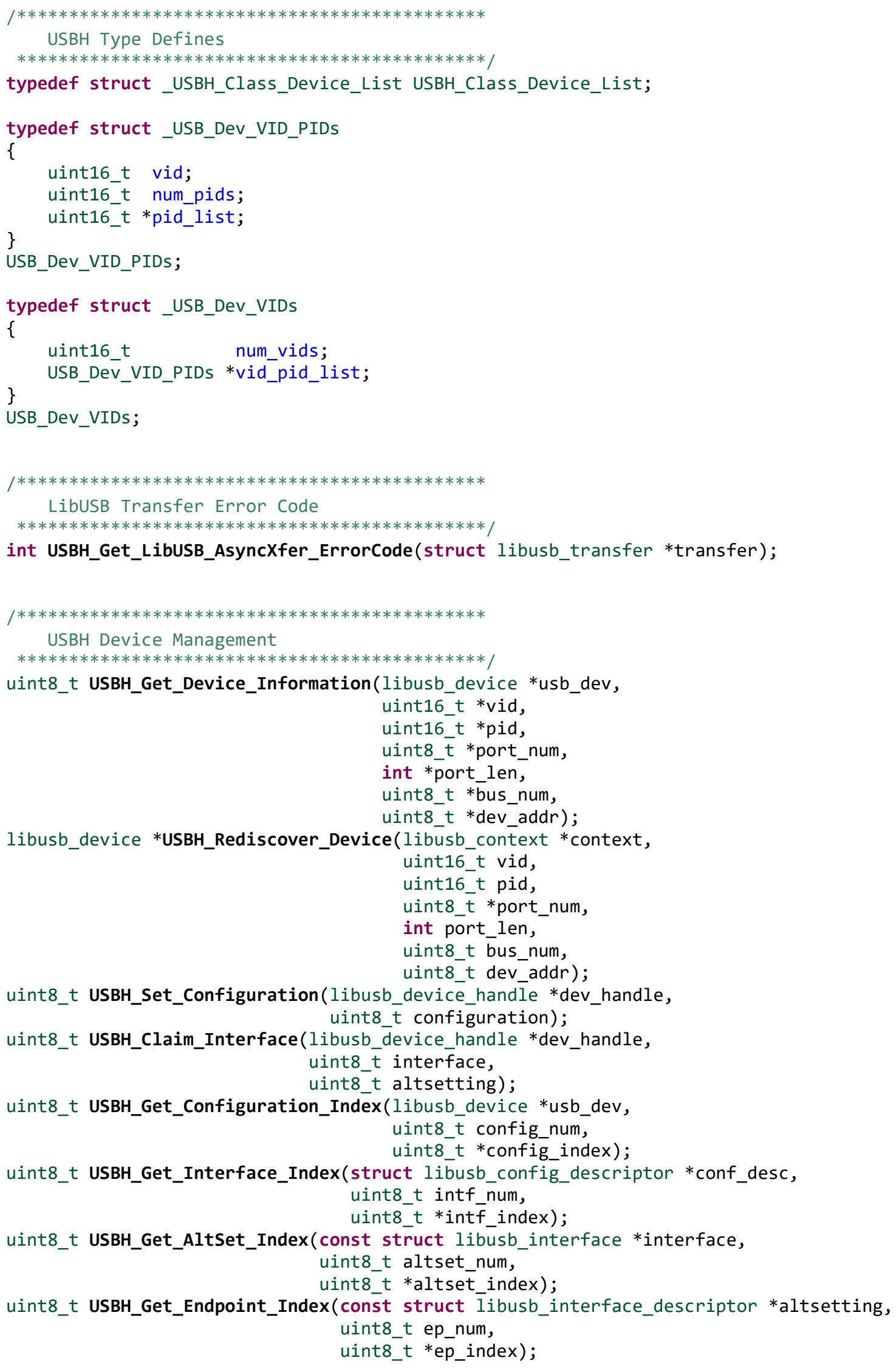




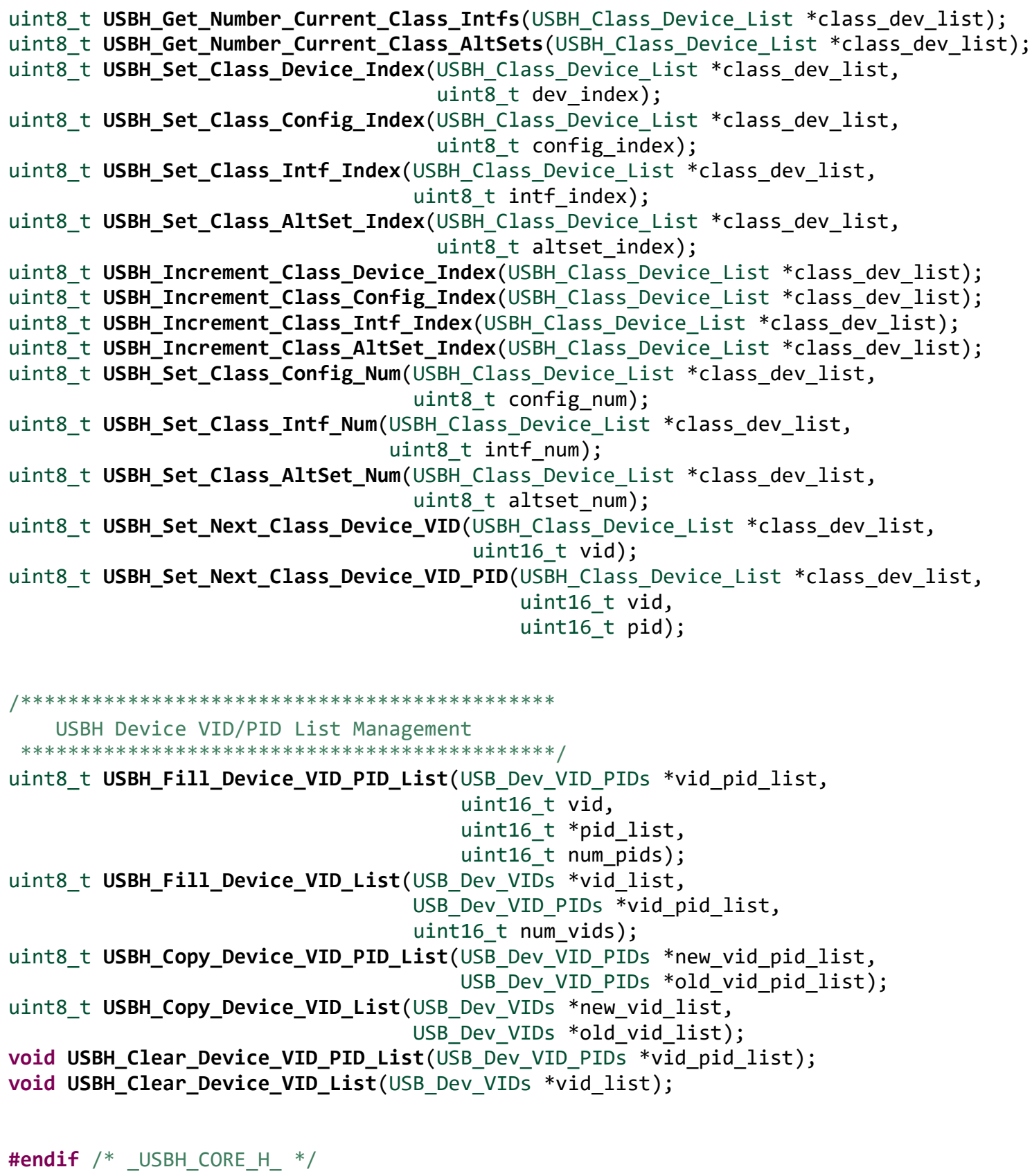




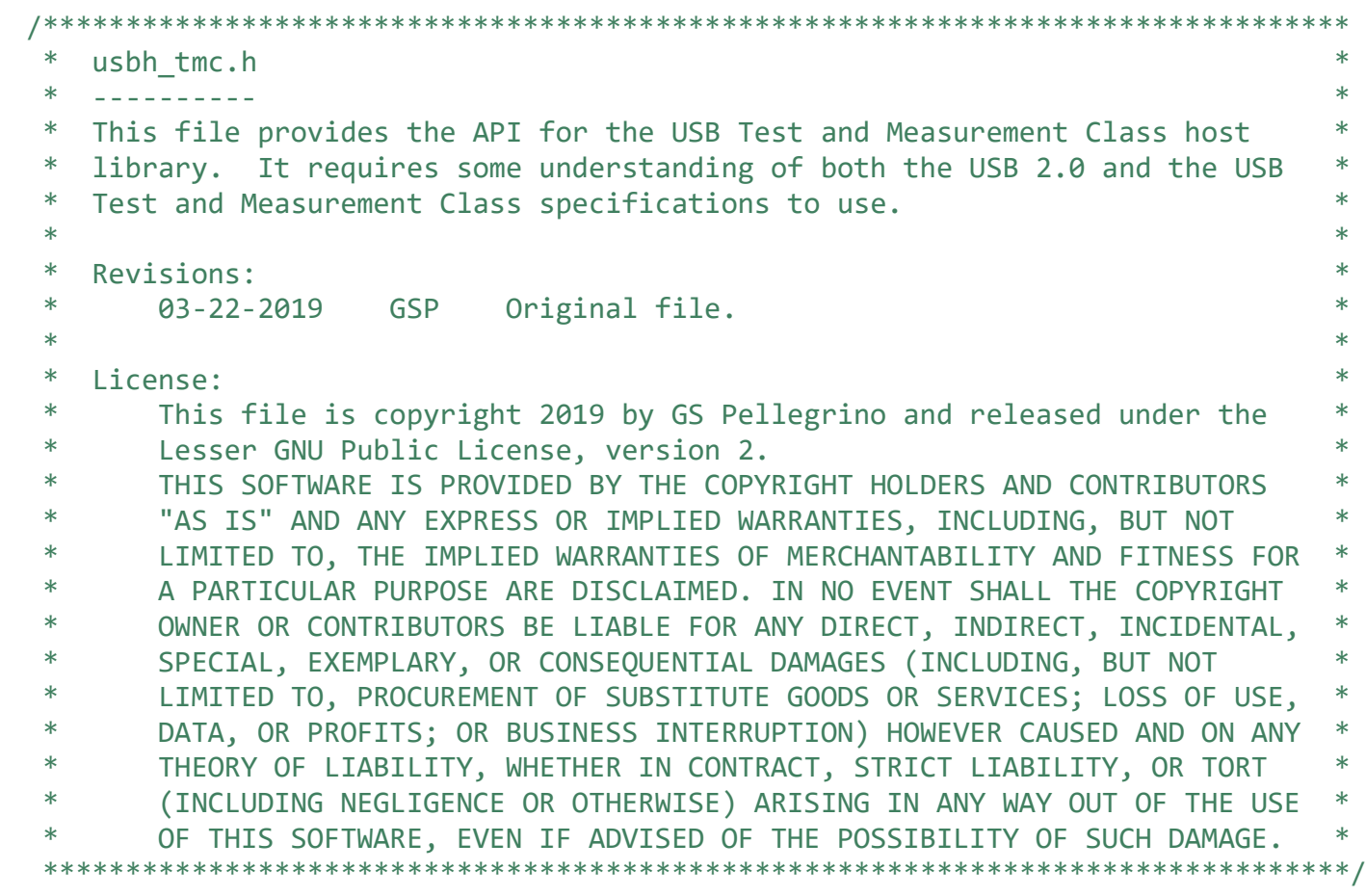

// Define to prevent recursive inclusion

\#ifndef _USBH_TMC_H_

\#define _USBH_TMC_H_

// Includes

\#include <stdint.h>

\#include "libusb.h"

\begin{tabular}{|c|c|}
\hline \multicolumn{2}{|c|}{$\begin{array}{l}\text { USBTMC Class Defines } \\
* * * * * * * * * * * * * * * * * * * * * * * * * * * * * * * * * * * * * * * * * * * * * /\end{array}$} \\
\hline \#define USBTMC_CLASS & $0 \times \mathrm{FE}$ \\
\hline \#define USBTMC_SUBCLASS & $0 \times 03$ \\
\hline \#define USBTMC_PROTOCOL_NO_SUBCLASS & $0 \times 00$ \\
\hline \#define USBTMC_PROTOCOL_USB448 & $0 \times 01$ \\
\hline
\end{tabular}

\#define USBTMC_HDR_SIZE

12 


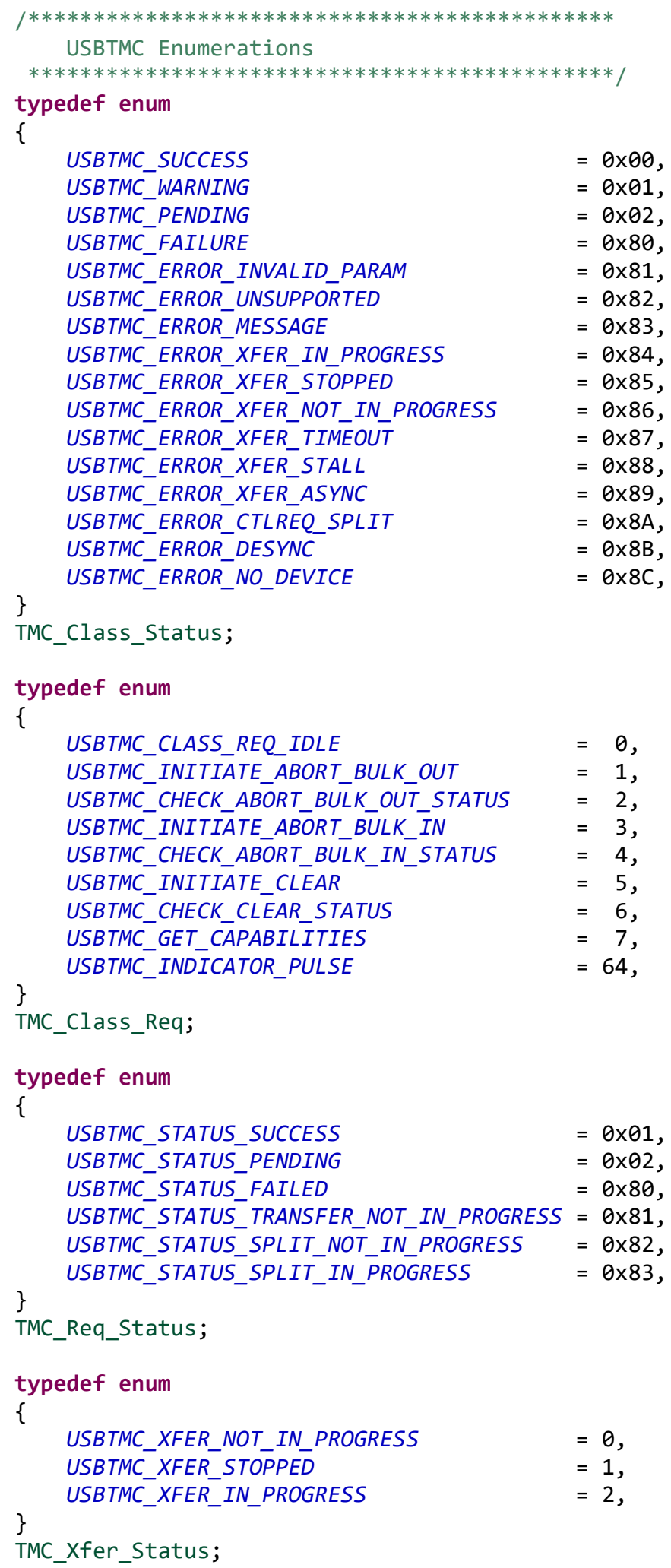




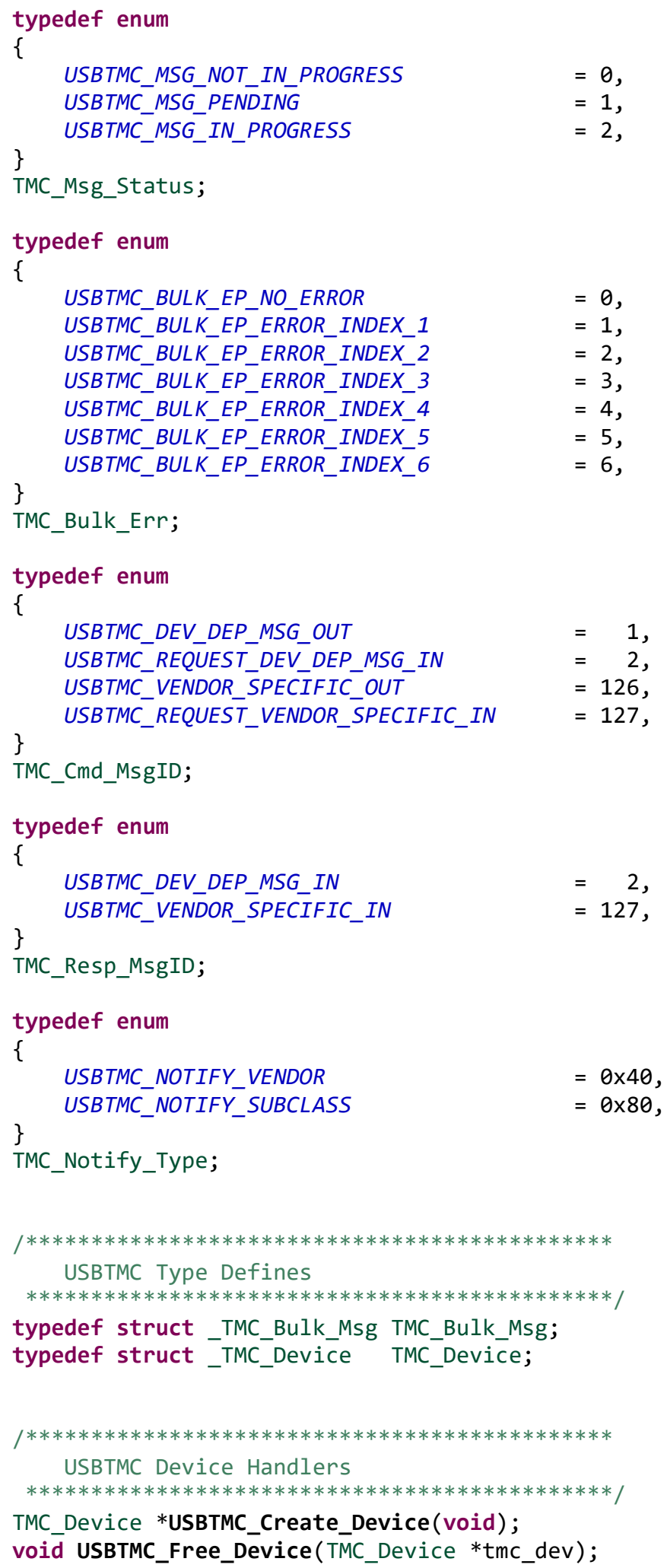




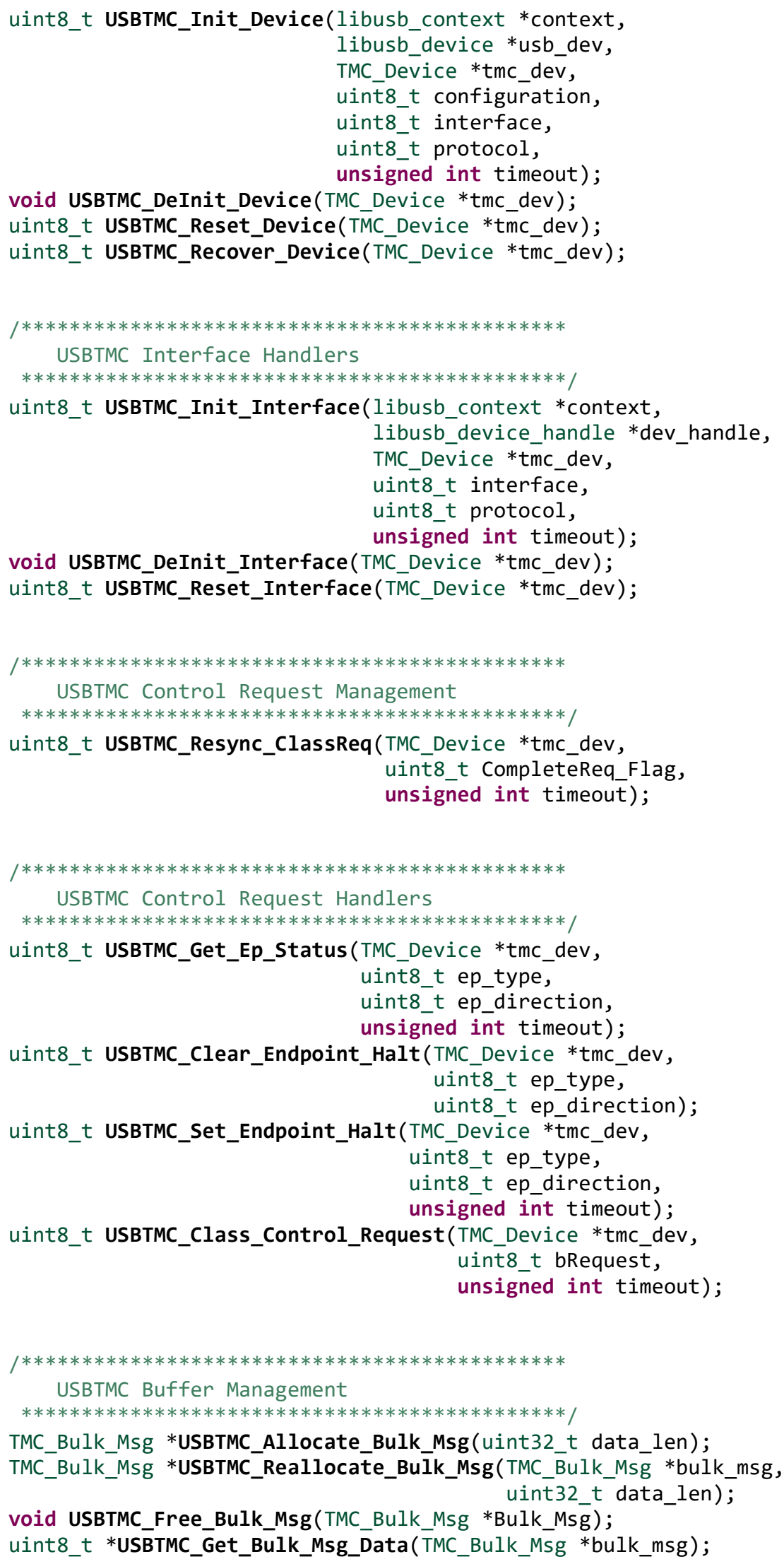




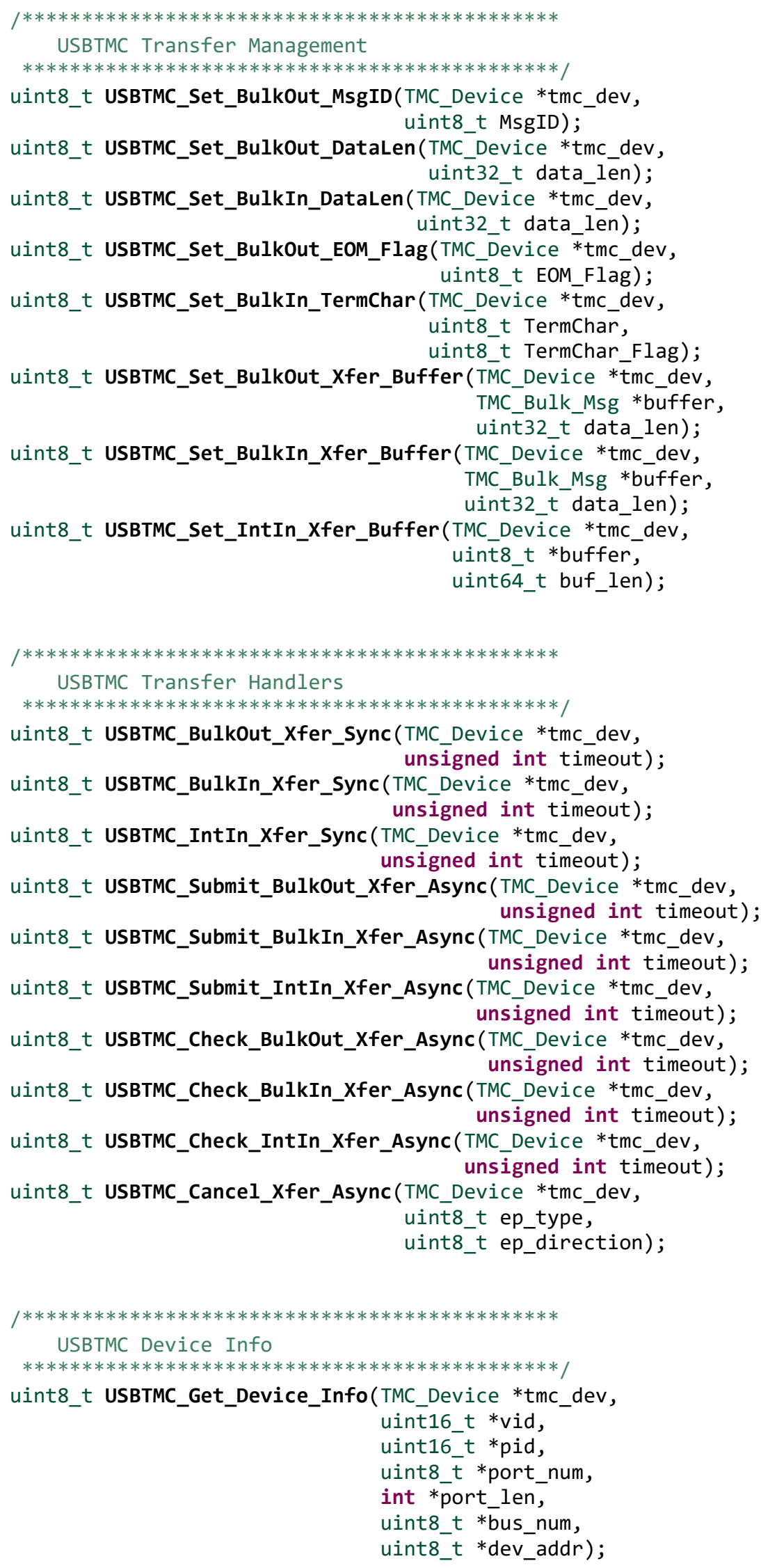




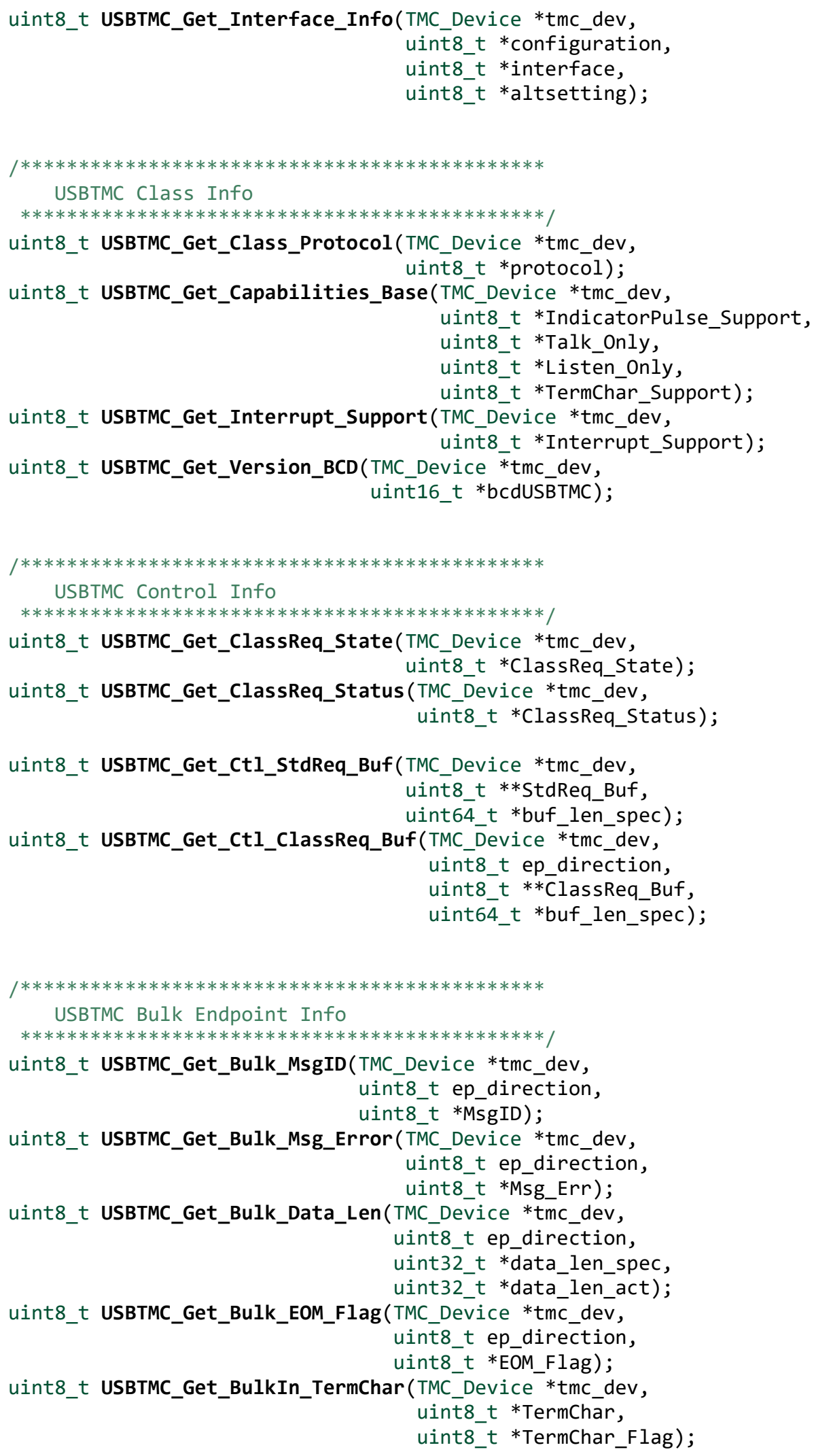




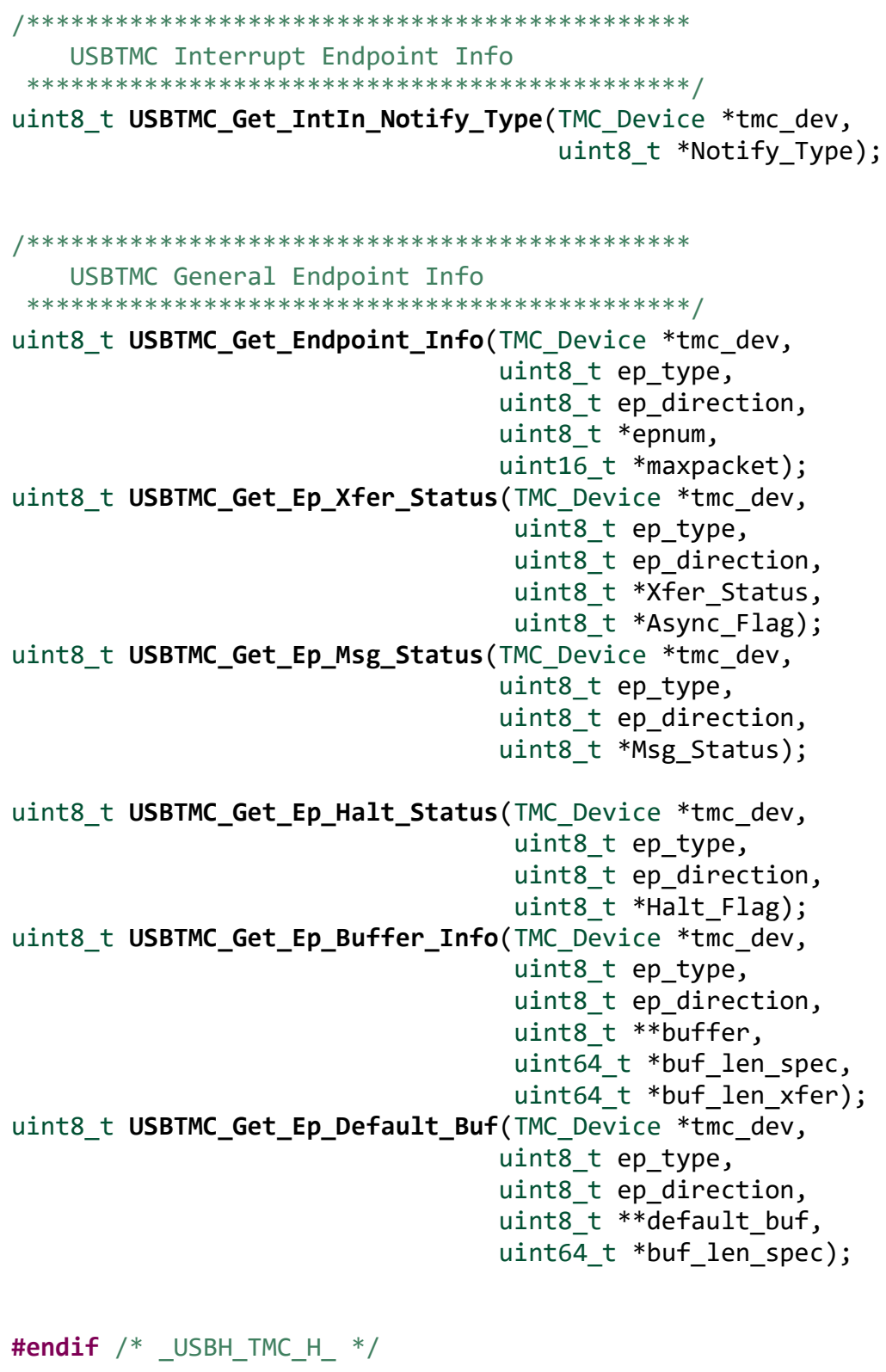

\#endif /* USBH_TMC_H_ $*$ / 


\section{Appendix F: MATLAB Rotordynamics Functions}

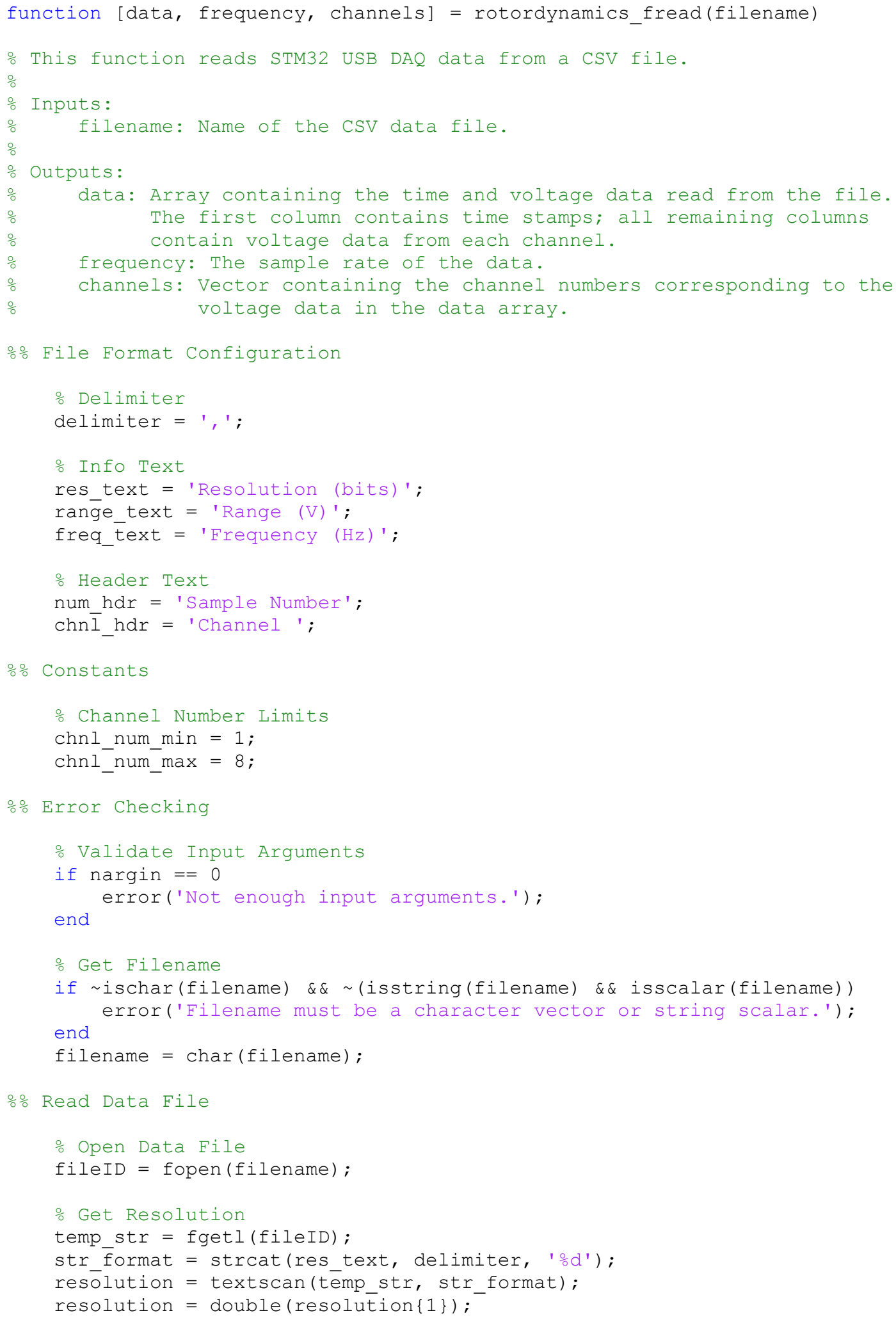




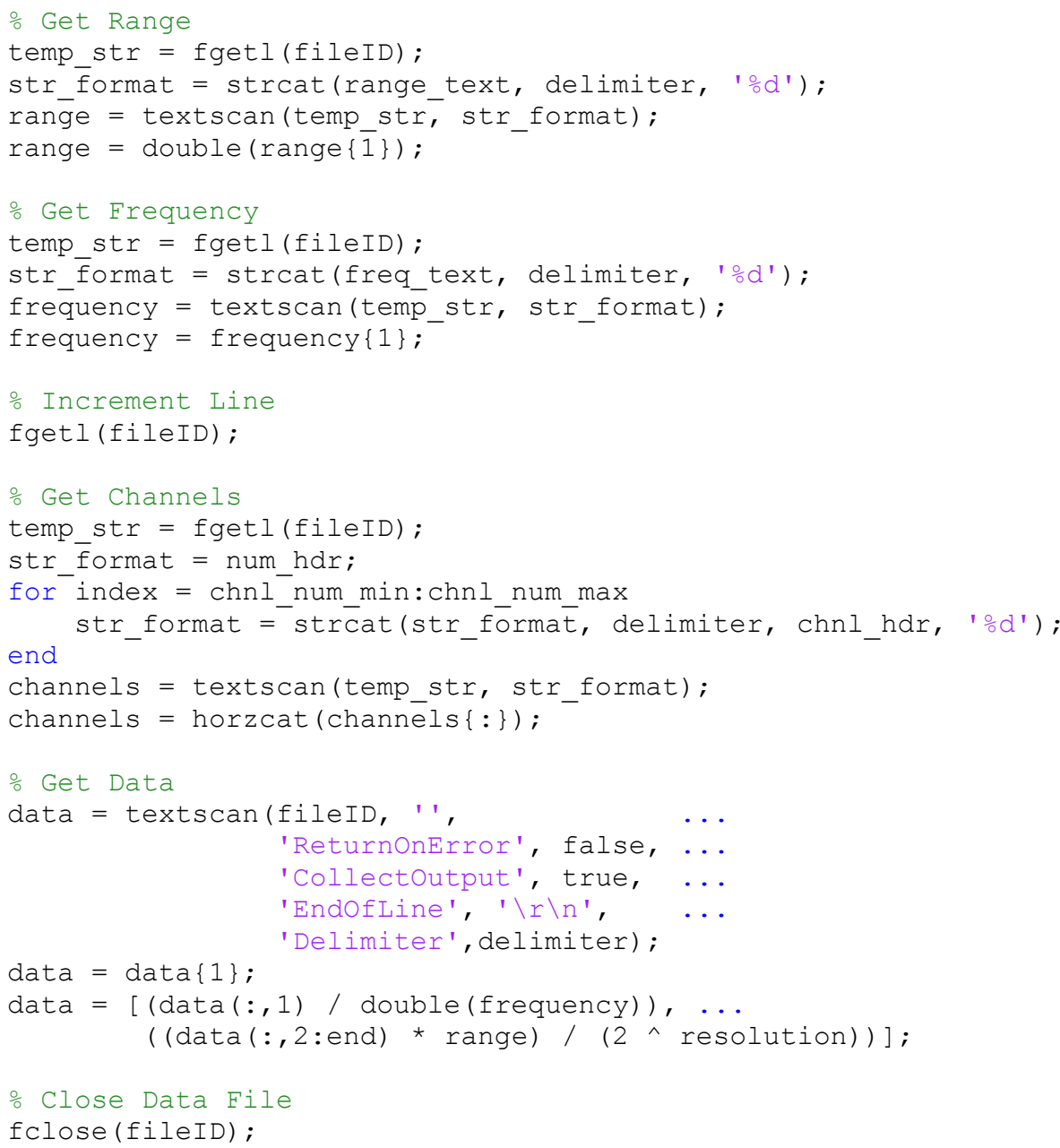

end 


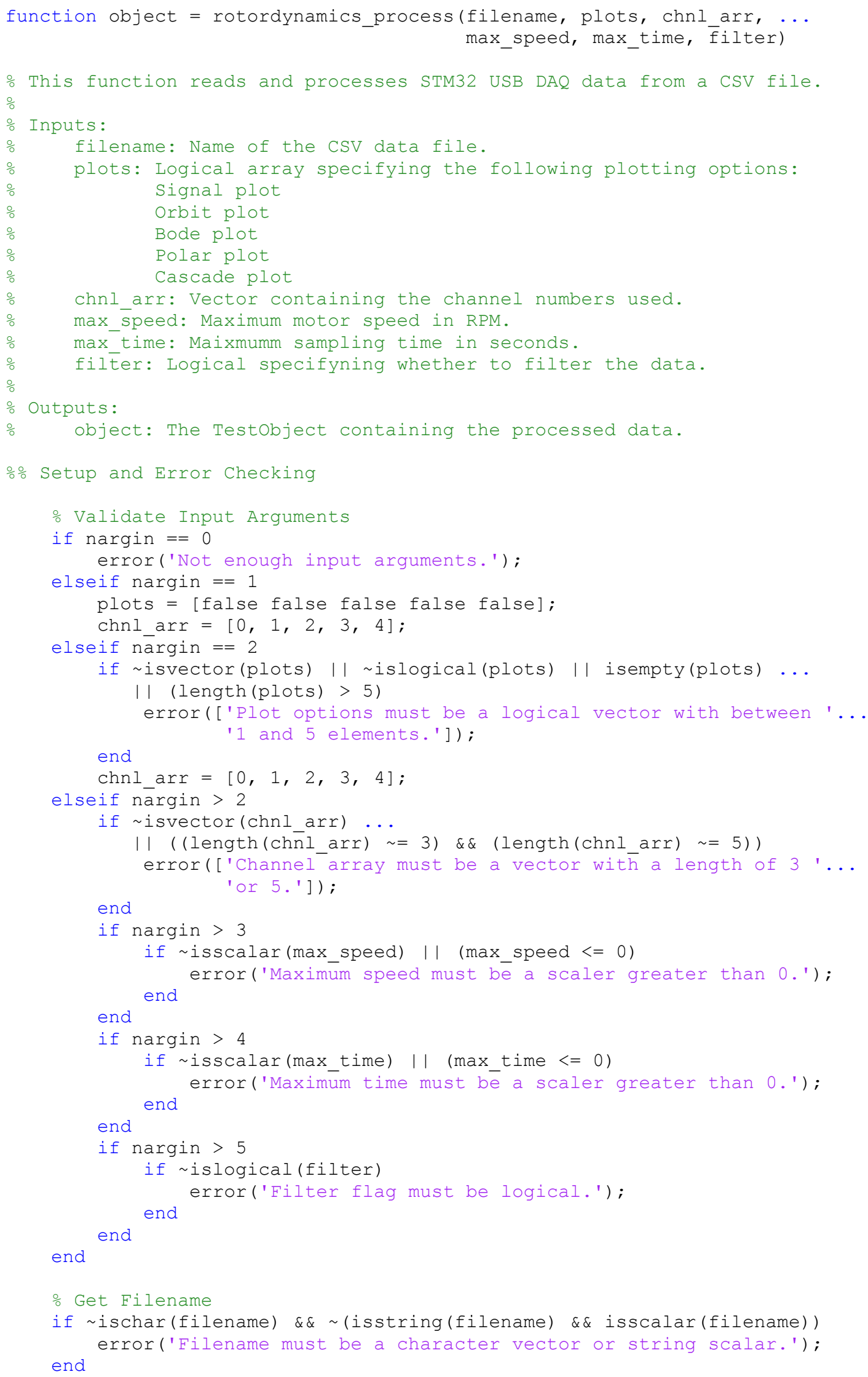


filename $=\operatorname{char}(\mathrm{filename})$;

응 Read and Process Data

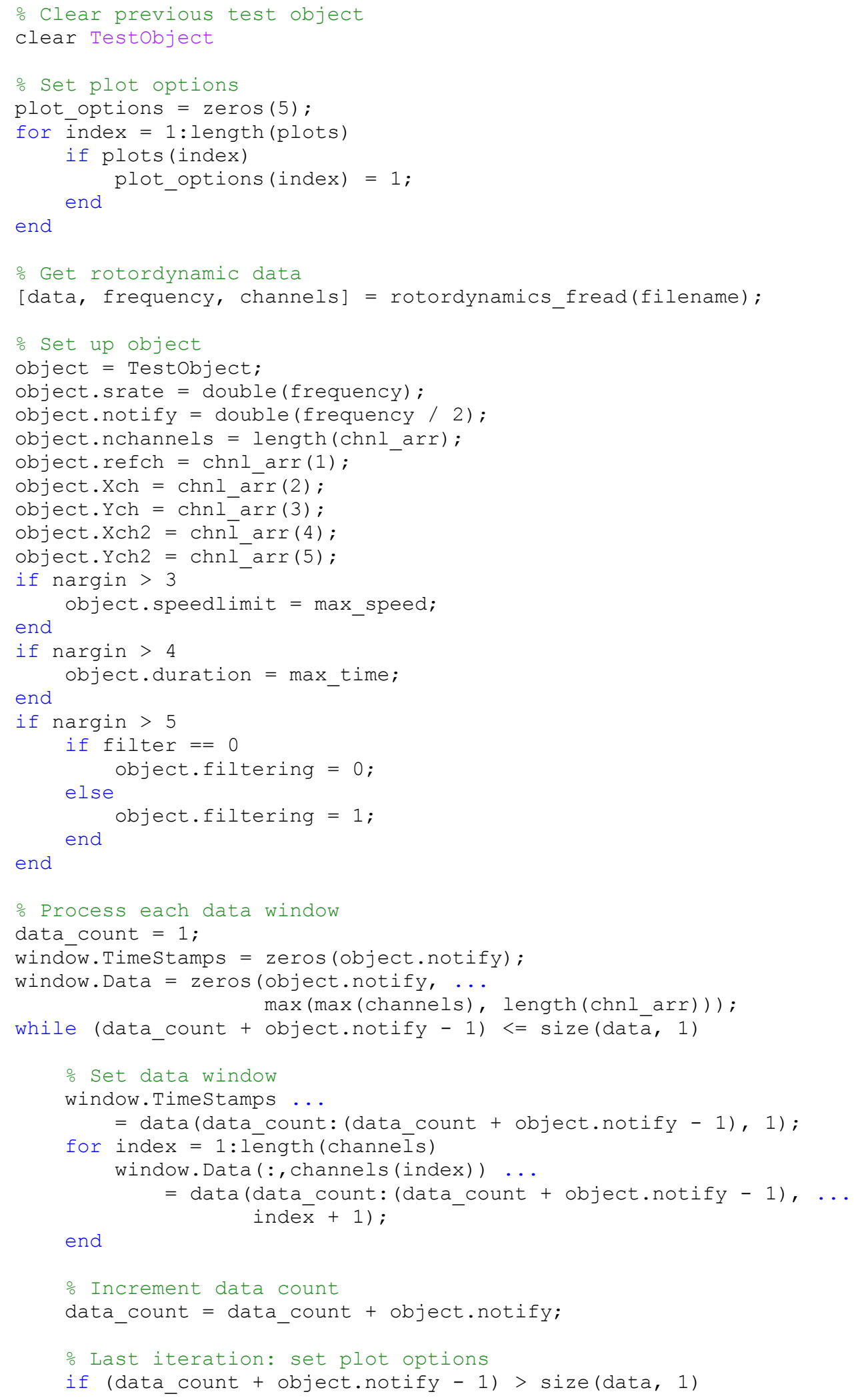




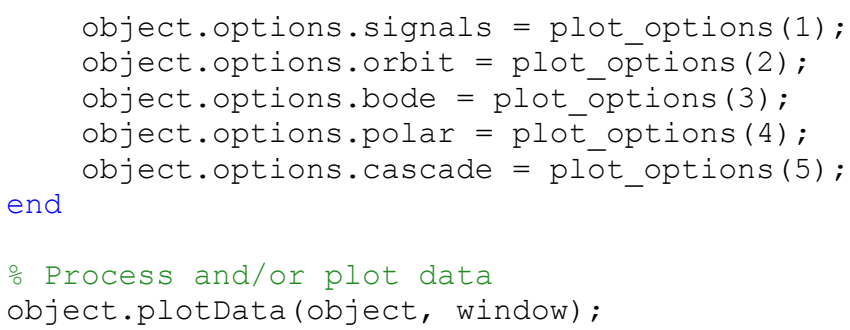




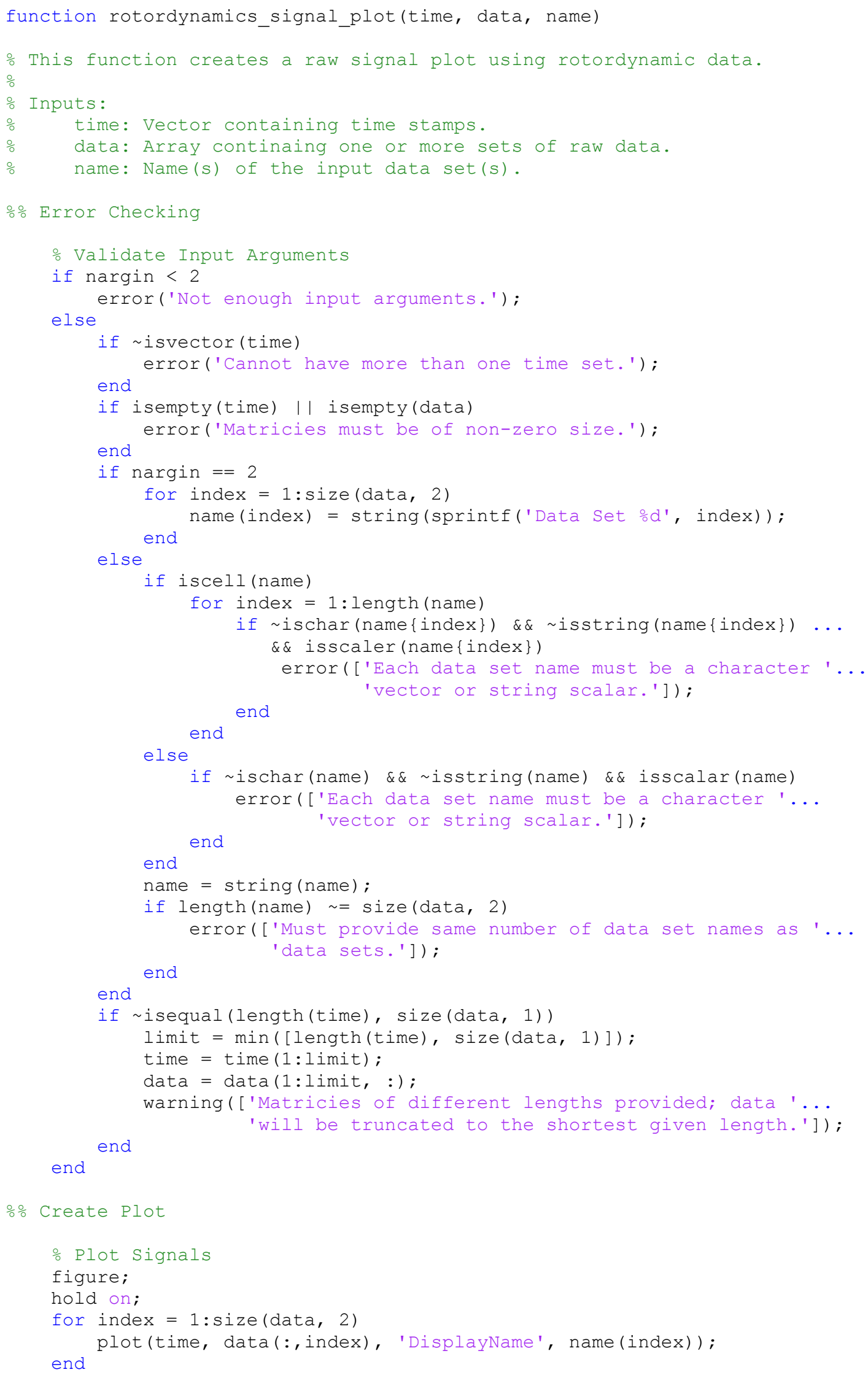


hold off;

title('Channel Signals');

xlabel ('Time (sec)');

ylabel ('Voltage (V)');

if nargin $==3$

legend ('Show') ;

end

end 


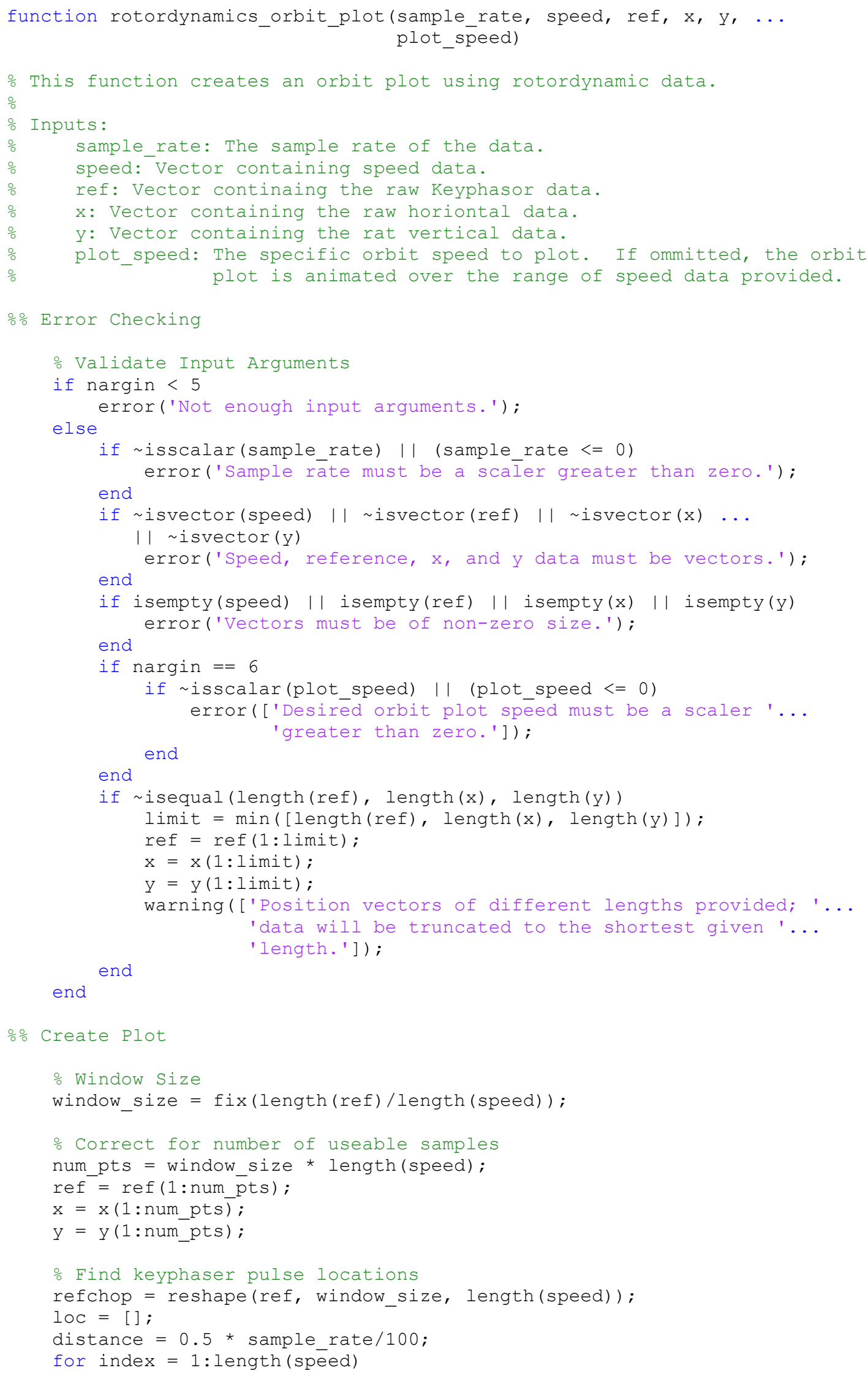




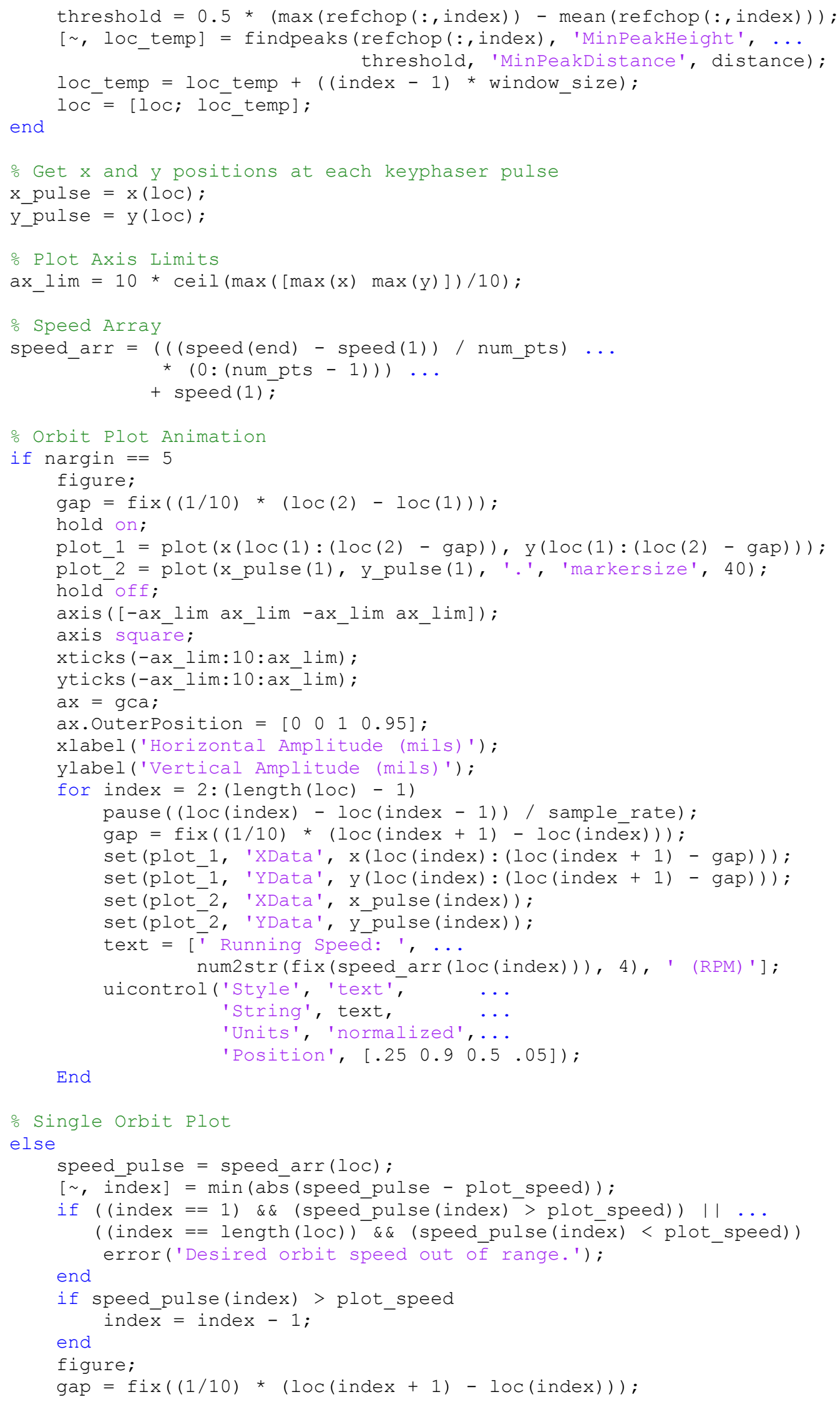




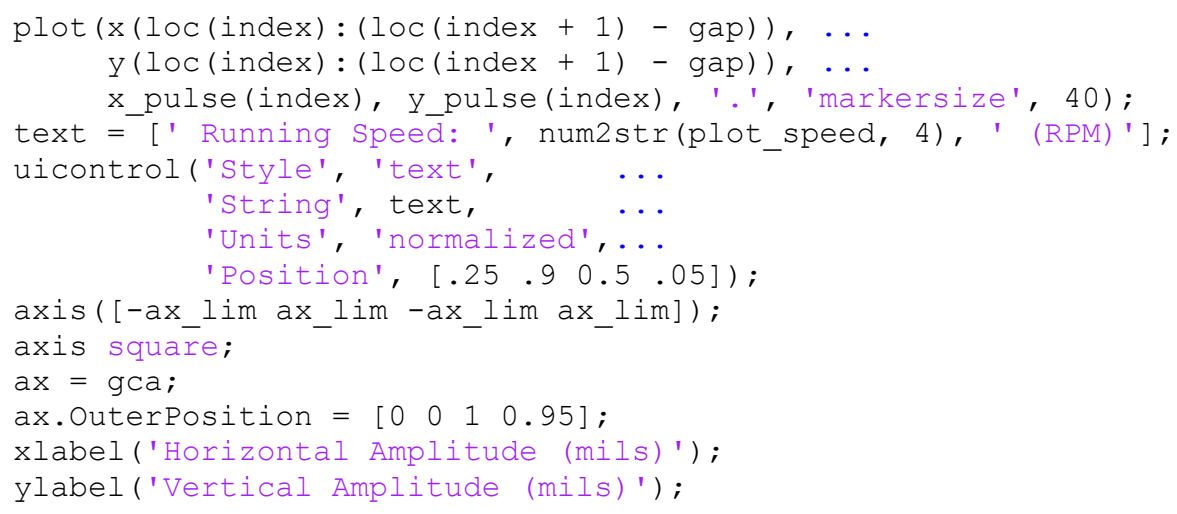




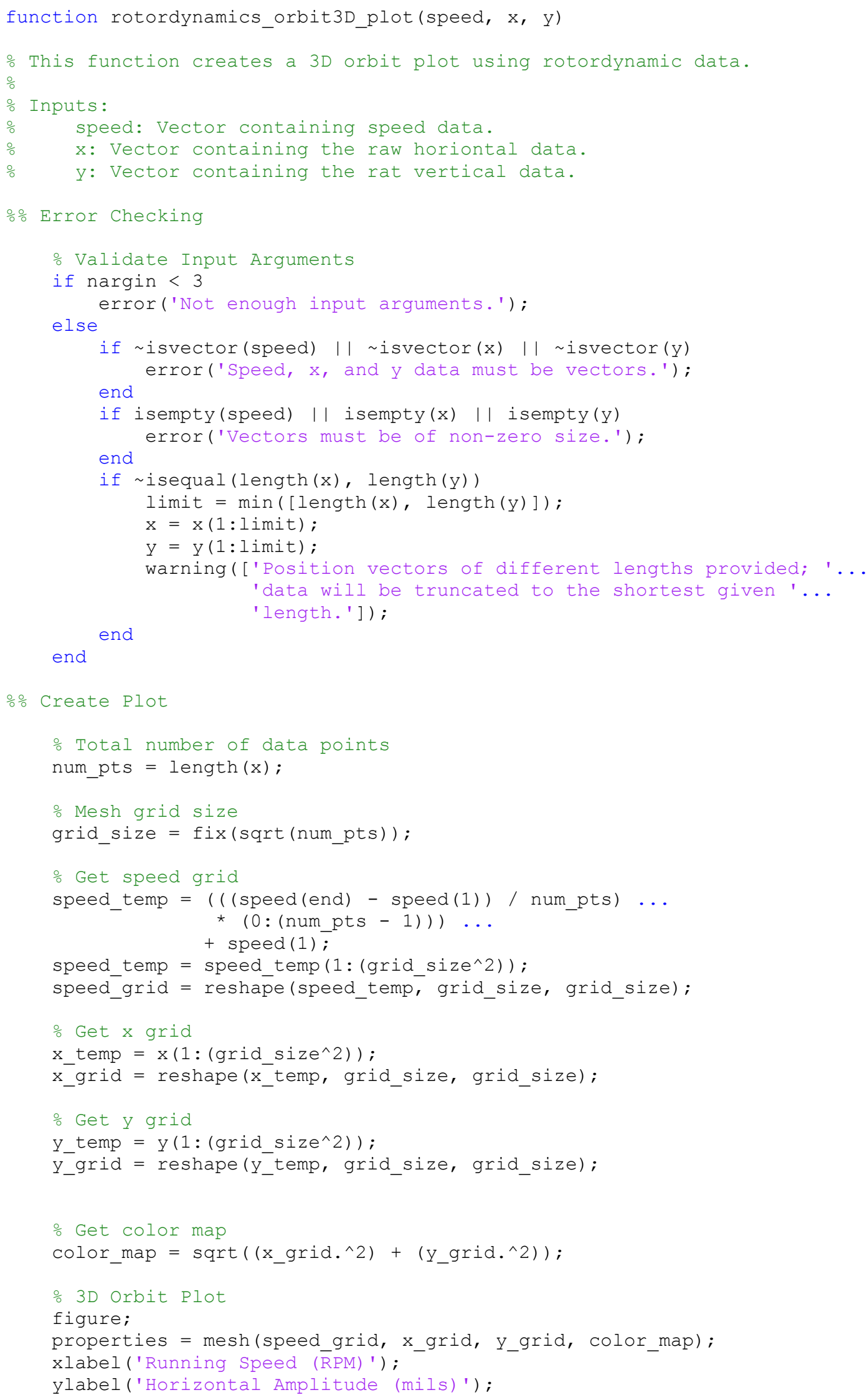


zlabel('Vertical Amplitude (mils)');

properties.FaceColor = 'none';

properties.Meshstyle = 'column';

end 


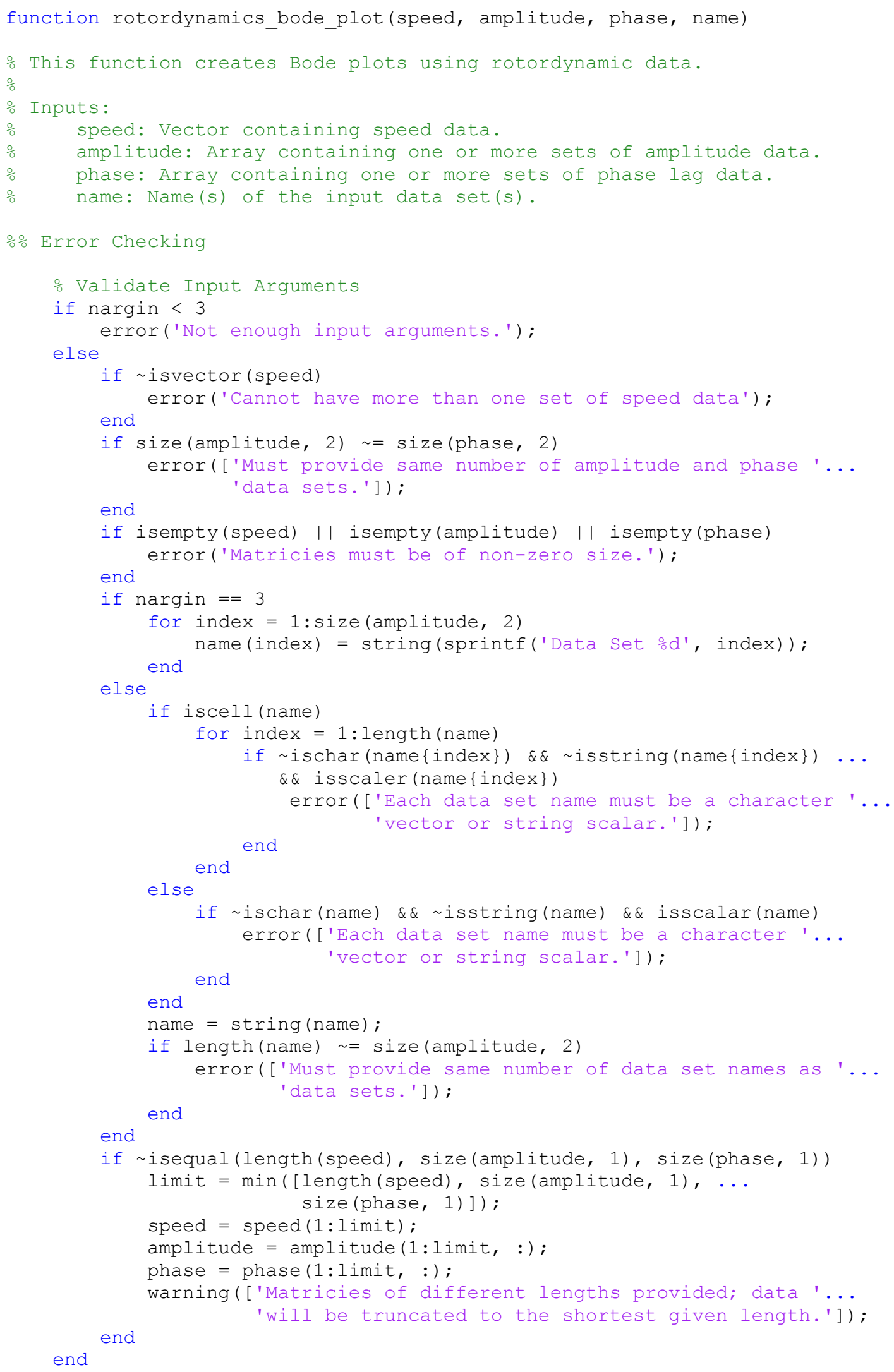




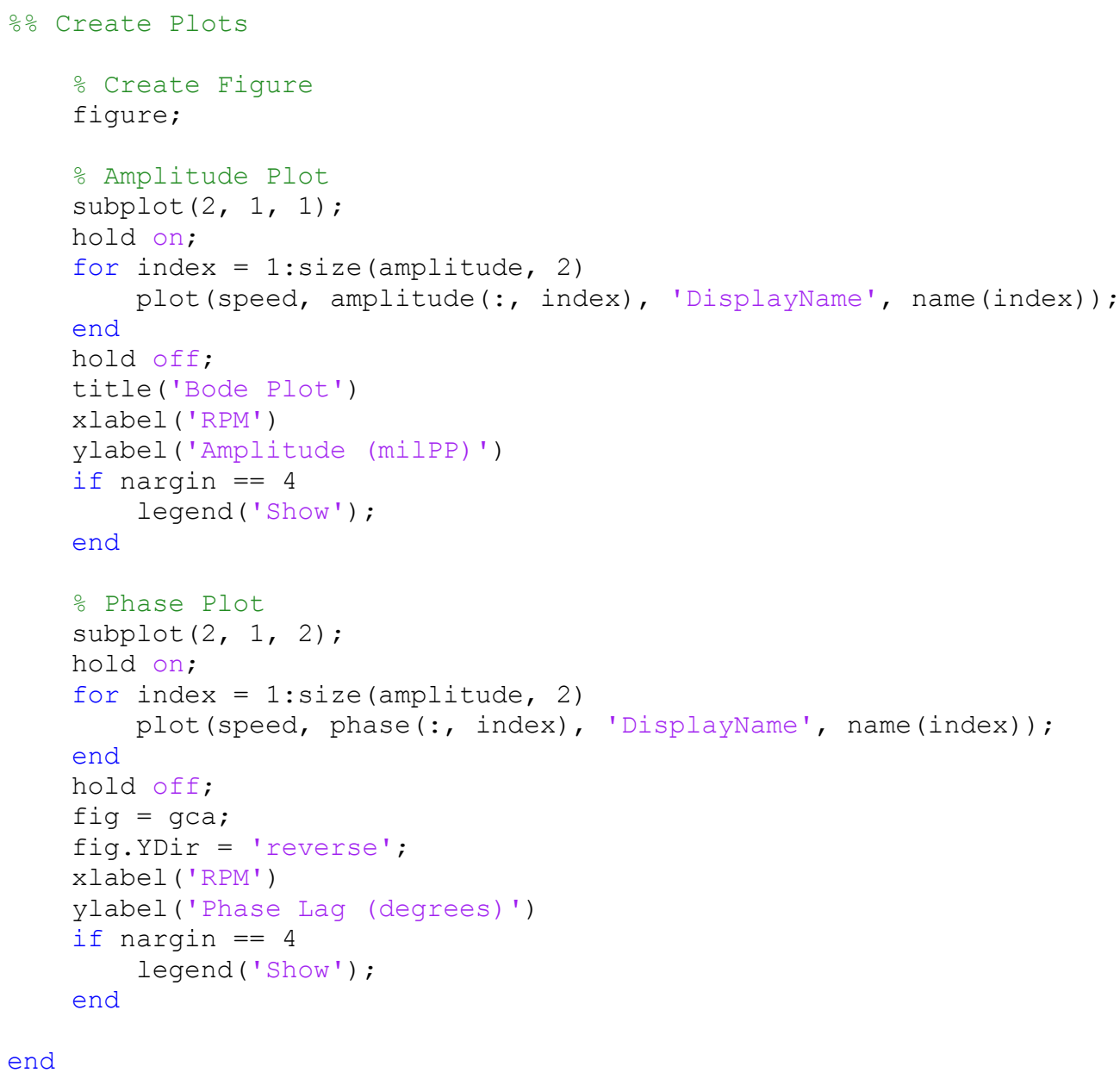




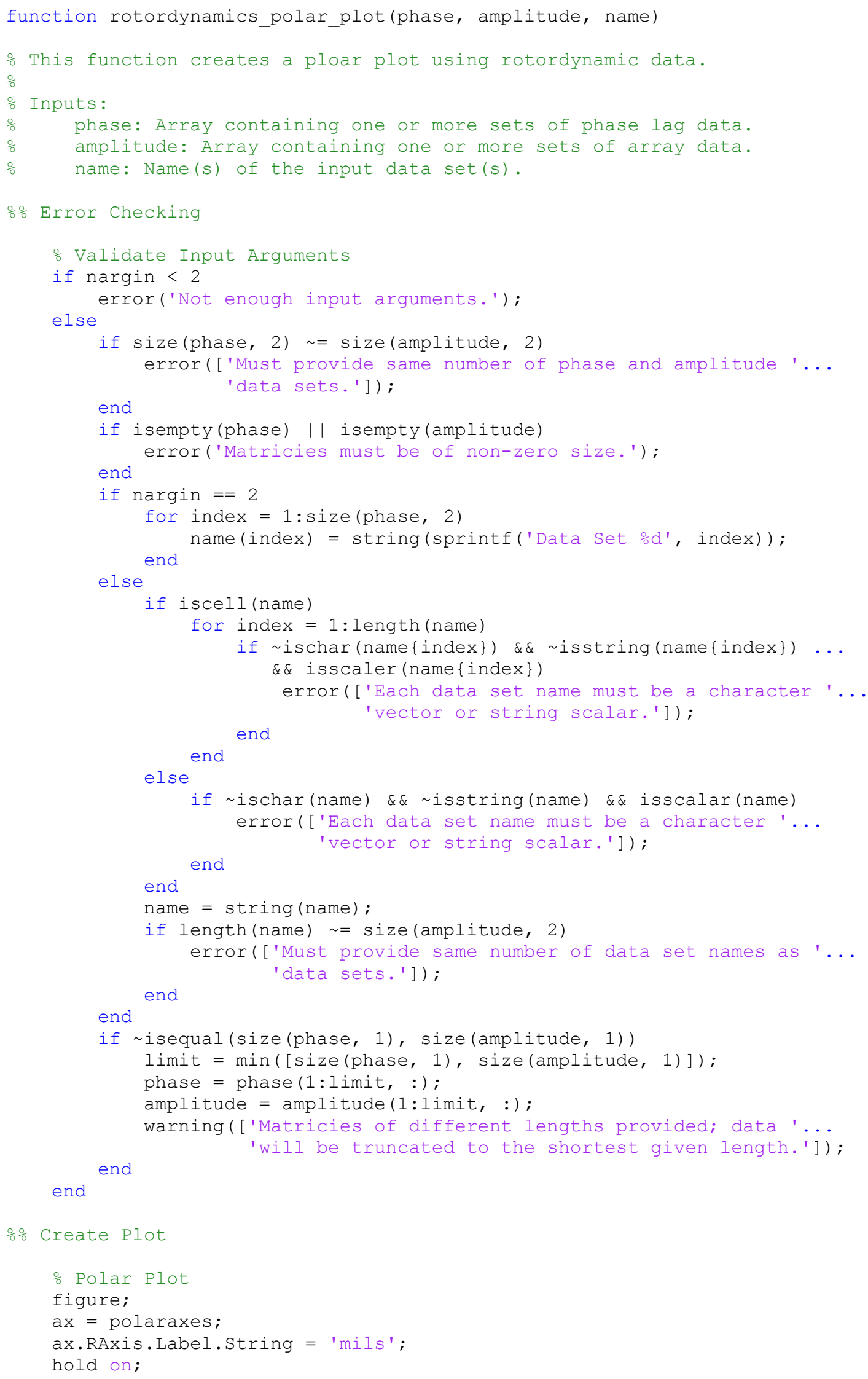


for index = 1:size (phase, 2)

polarplot(phase(:, index), amplitude(:, index), 'Displayname',...

end name (index)) ;

hold off;

if nargin $==3$

end

end 


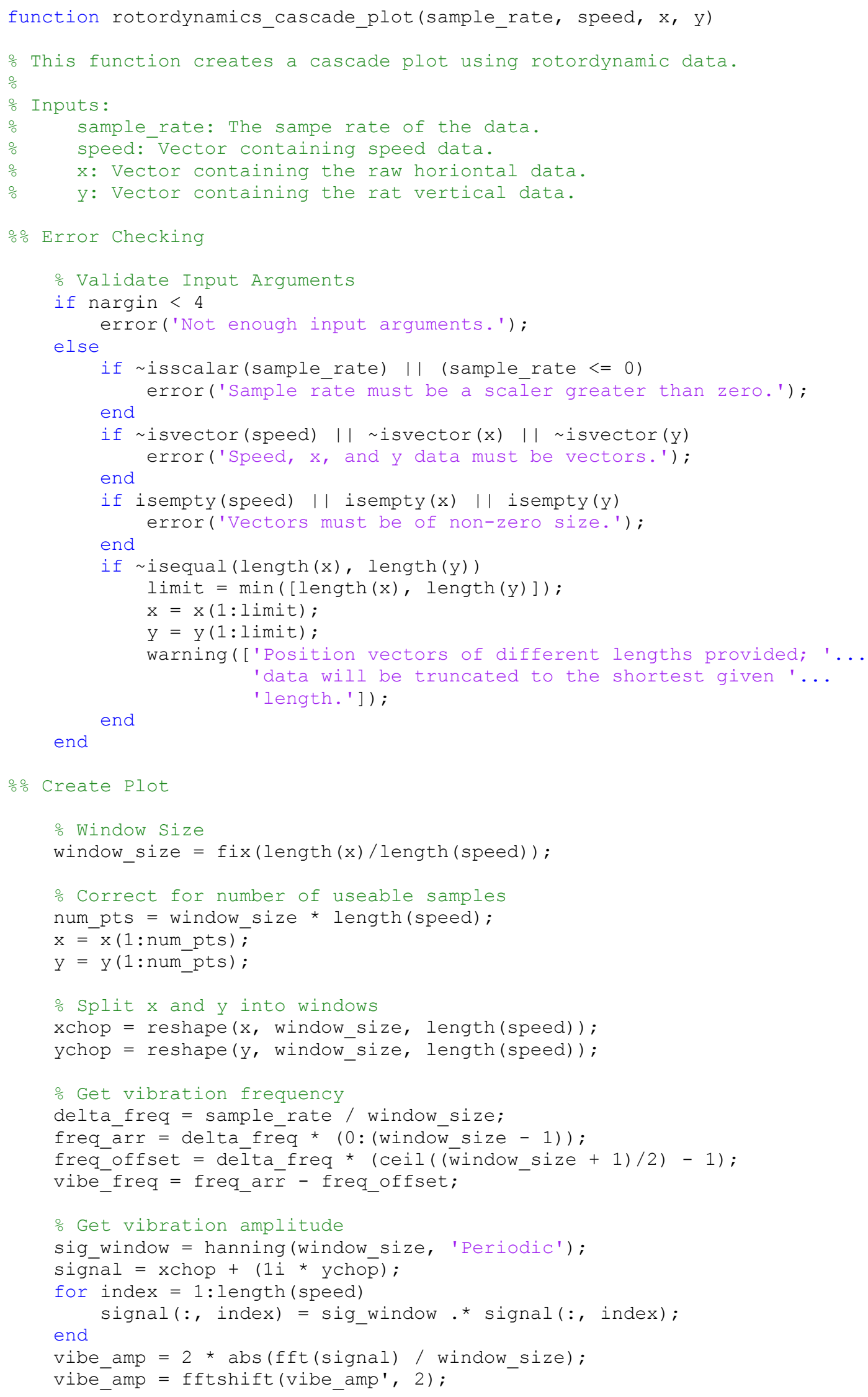


- Cascade Plot

figure;

waterfall(vibe_freq, speed, vibe_amp)

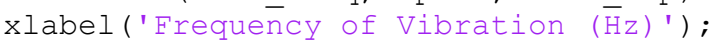

ylabel ('Running speed (RPM)');

zlabel('Amplitude of Vibration (mils)');

$\operatorname{axis}([-100100$-inf inf 0 inf]);

end 


\section{Appendix G: STM32 USB Data Acquisition System Task and State Diagrams}

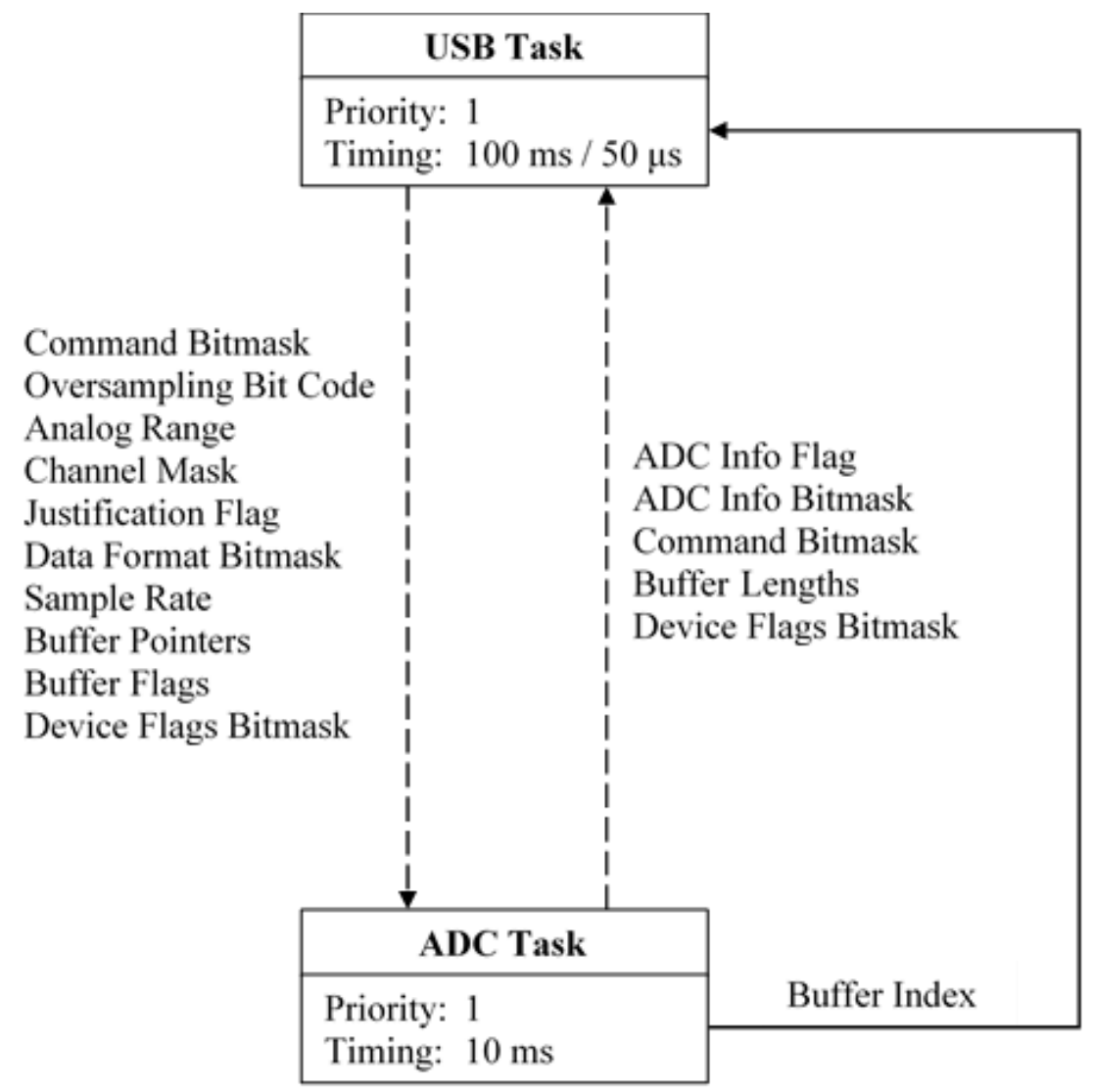

Figure G1. STM32 USB Data Acquisition System task diagram. The USB task has two timing requirements dependent on the state it is in. 


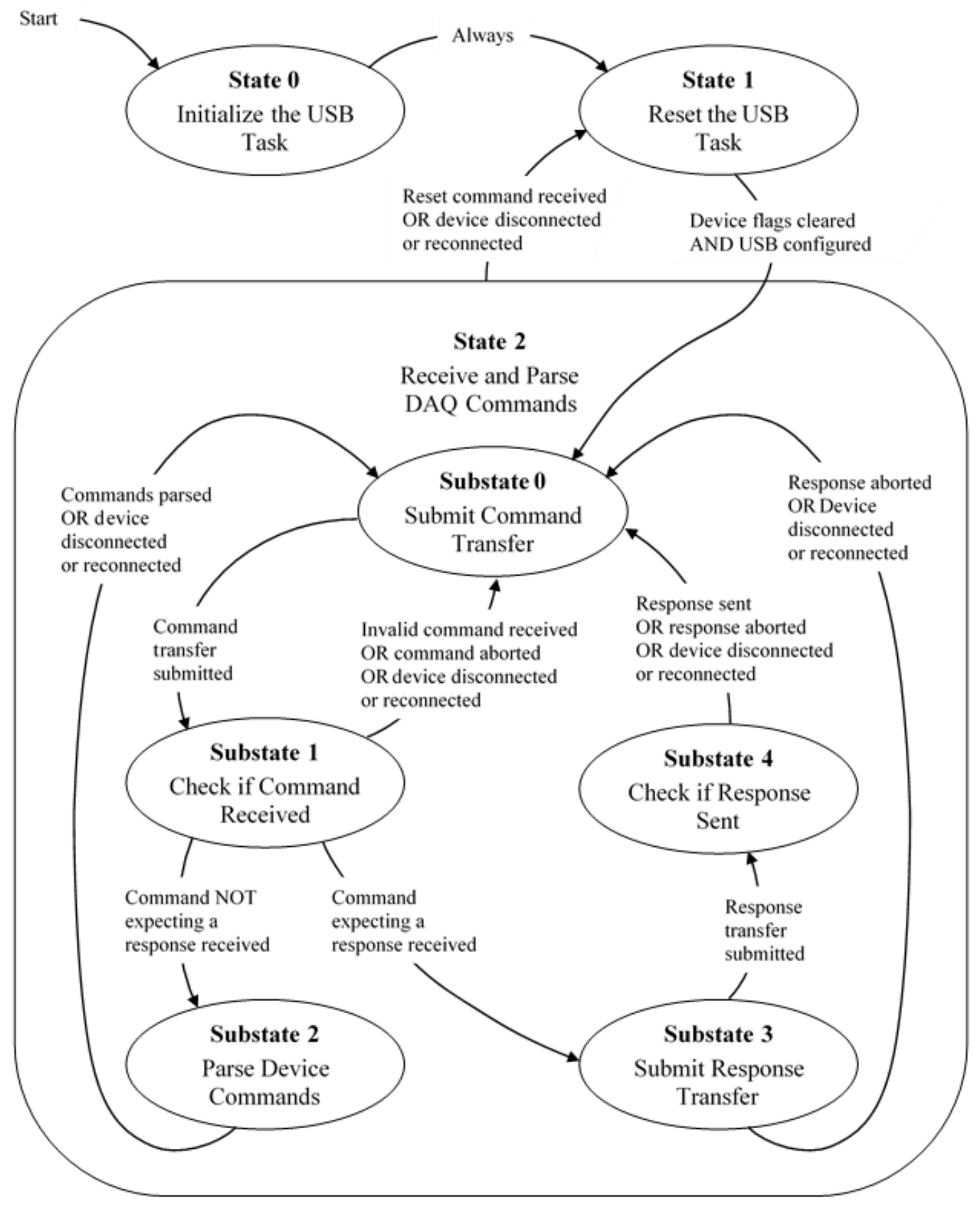

Figure G2. STM32 USB Data Acquisition System USB task state diagram. States 0 and 1 have a timing requirement of $100 \mathrm{~ms}$, while state 2 has a timing requirement of $50 \mu \mathrm{s}$. 


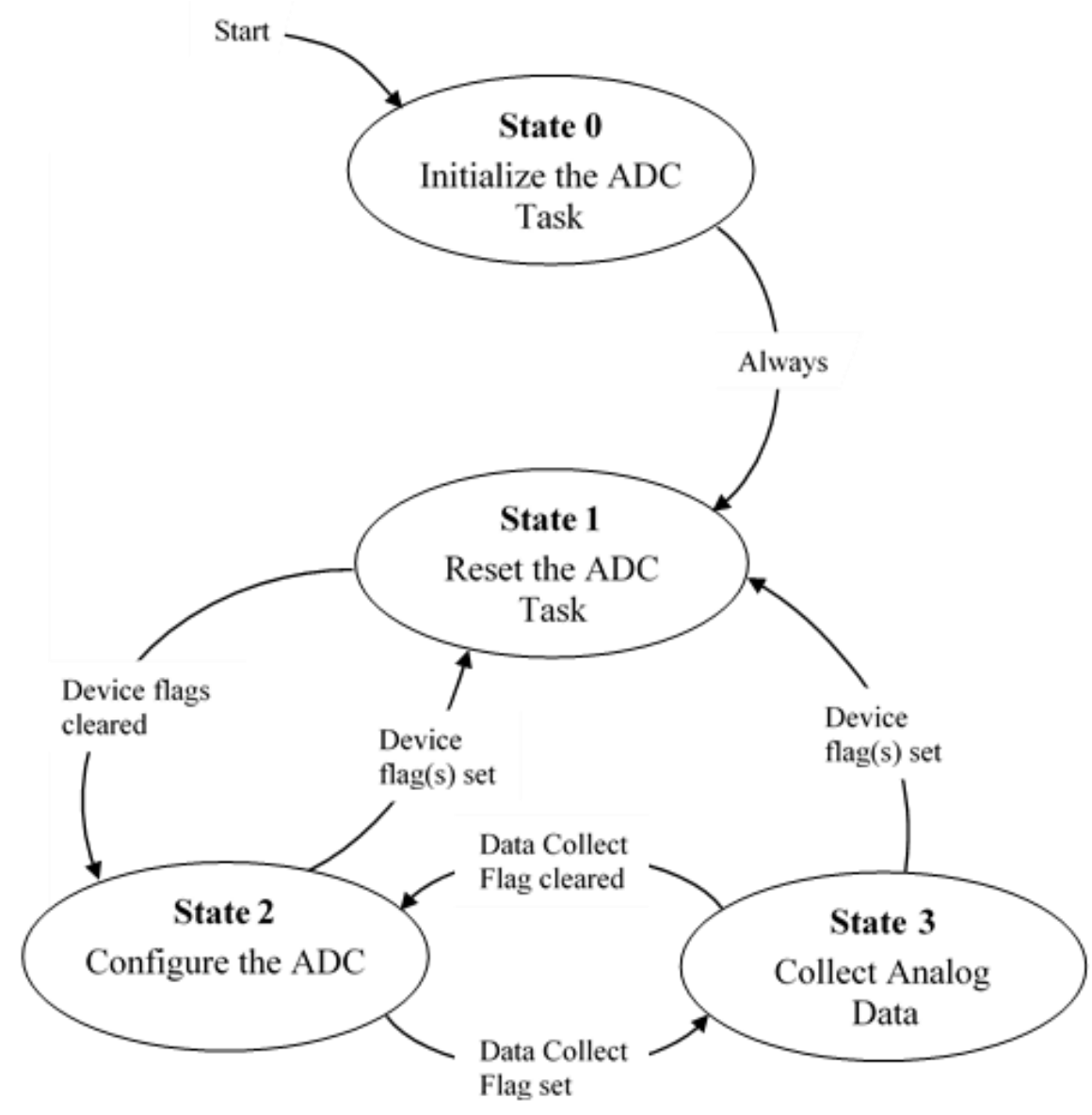

Figure G3. STM32 USB Data Acquisition System ADC task state diagram. 


\section{Appendix H: How to Use the STM32 USB Data Acquisition System}

Before collecting data, it is important that both the STM32 USB Data Acquisition System and the host computer are configured properly. The following steps should be taken to set up the STM32 USB DAQ:

1. Check the compatibility of the selected Nucleo board with the Data Acquisition Expansion Board and make any necessary or desired changes to the expansion board.

2. Modify the system core clock as appropriate. The internal USB clock source should be set to $48 \mathrm{MHz}$ for a full-speed connection.

3. Modify the USB and ADC board support packages as appropriate. Make sure to enable or disable the ADC GPIO pins in accordance with the expansion board configuration.

4. Program the Nucleo board if any code changes have been made.

It should be noted that the Data Acquisition Expansion Board, in its default configuration, is designed to work with the Nucleo-F446RE development board. Although its design allows the expansion board to be configured for use with other Nucleo boards, the performance and compatibility of these other boards is not guaranteed. The appropriate data sheet for the selected development board should be consulted before trying to use it with the expansion board.

In addition to configuring the STM32 USB DAQ, it is necessary to make sure the host computer is configured to run the console application. To do so, the following steps should be taken: 
1. Check if the computer supports POSIX threads. If it does not, download and install an appropriate POSIX threads library. For windows computers, the appropriate version of MinGW-64 can be installed to provide support for POSIX threads.

2. If necessary or desired, modify and recompile the console application.

3. If necessary, install a driver to communicate USBTMC devices. It may be necessary to connect the STM32 USB DAQ to the computer do so. For Windows based computers, Zadig can be used to install a generic USB driver.

Once both the STM32 USB DAQ and the host computer are configured for use, data can be collected. To do so, first connect the STM32 USB DAQ to the computer and connect any necessary sensor inputs to the DAQ. Then begin the console application and follow the on-screen prompts. The prompts, as seen in Figure H1, are as follows:

1. Select a USB device number from the provided list.

2. Enter the desired device configuration and interface numbers to use.

3. Enter the channel number(s) to use. The available channel numbers range from 1 to either 4,6 , or 8 , depending on the particular ADC used. Channel numbers should be separated by a space, comma, semicolon, period, or tab; other delimiters result in an error. Channel numbers should not be repeated.

4. If prompted, select the analog range. Acceptable inputs are 5 for a \pm 5 volt range, or 10 for a \pm 10 volt range.

5. If prompted, enter the oversampling ratio. Acceptable inputs are 0 (same as 1), 1, $2,4,8,16,32$, or 64 . Note that the oversampling ratio affects the maximum possible sampling rate; refer to the appropriate AD760x data sheet for details. 
6. Enter the sampling rate. This can be any number in a range of 1 to 200,000 or 300,000 , depending on the particular ADC used. Note that high sampling rates may result in a loss of data due to limitations of the DAQ or the USB full-speed connection. Additionally, sampling rates that do no align with the internal ADC timer of the DAQ may result in an inaccurate sampling rate. The DAQ should be tested for valid sampling rates before collecting any data.

7. Begin collecting data by entering anything. There is no time limit on how long a user can wait before initiating data collection.

8. Enter ' $X$ ' (or ' $x$ ') to end data collection. There is no time limit on how long a user can collect data; however, if data is collected over a long period of time, the 32-bit sample number value may overflow, or the computer drive may not have enough space to save all data to the CSV.

9. Enter anything to exit the program. After the program exits, the data is ready to be read from the CSV file and processed.

Once data has been collected and saved to a CSV file, it can be read into MATLAB using either the rotordynamics_fread function to read in raw data, or the rotordynamics_process function to read and process the data. The processed data can then be plotted using the following functions:

- rotordynamics_signal_plot: Plots raw voltage data.

- rotordynamics_orbit_plot: Creates either a static or animated orbit plot.

- rotordynamics_orbit3D_plot: Creates a 3D orbit plot.

- rotordynamics_bode_plot: Creates amplitude and phase Bode plots. 
- rotordynamics_polar_plot: Creates a polar plot.

- rotordynamics_cascade_plot: Creates a full spectrum cascade plot.

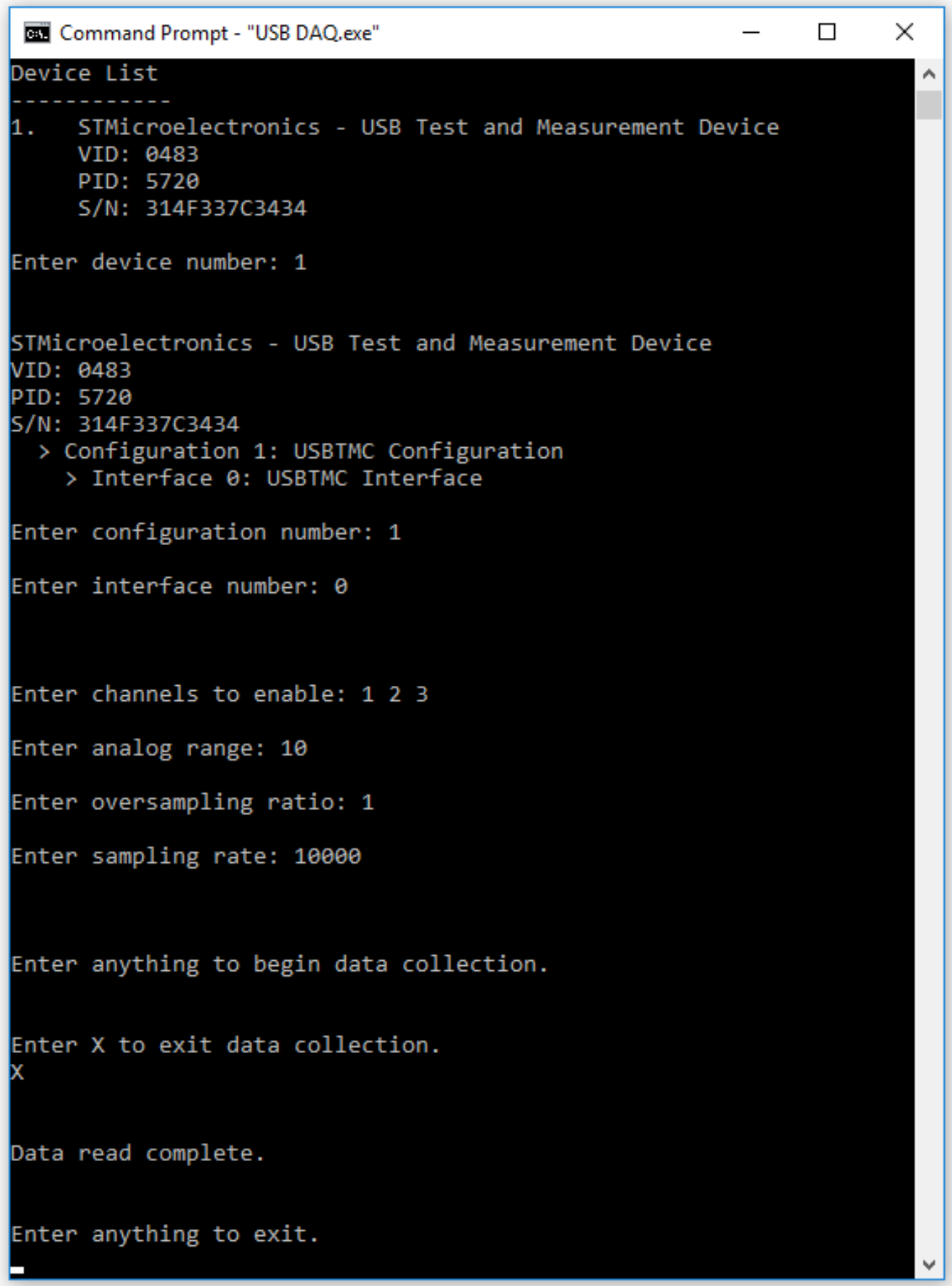

Figure H1. The console application used to collect data from the STM32 USB DAQ. 